Interdisciplinarity in

Translation and Interpreting

Process Research 


\section{Benjamins Current Topics}

ISSN 1874-0081

Special issues of established journals tend to circulate within the orbit of the subscribers of those journals. For the Benjamins Current Topics series a number of special issues of various journals have been selected containing salient topics of research with the aim of finding new audiences for topically interesting material, bringing such material to a wider readership in book format.

For an overview of all books published in this series, please see http://benjamins.com/catalog/bct

\section{Volume 72}

Interdisciplinarity in Translation and Interpreting Process Research Edited by Maureen Ehrensberger-Dow, Susanne Göpferich and Sharon O’Brien These materials were previously published in Target 25:1 (2013) 


\section{Interdisciplinarity in Translation and Interpreting Process Research}

Edited by

Maureen Ehrensberger-Dow

Zurich University of Applied Sciences

Susanne Göpferich

Justus Liebig University Giessen

Sharon O'Brien

Dublin City University

John Benjamins Publishing Company

Amsterdam / Philadelphia 
The paper used in this publication meets the minimum requirements of the American National Standard for Information Sciences - Permanence of Paper for Printed Library Materials, ANsi z39.48-1984.

DOI $10.1075 /$ bct.72

Cataloging-in-Publication Data available from Library of Congress: LCCN 2015009723 (PRINT) / 2015012896 (E-BOOK)

ISBN 978902724260 O (HB)

ISBN 9789027268488 (Е-BOOK)

(C) 2015 - John Benjamins B.V.

No part of this book may be reproduced in any form, by print, photoprint, microfilm, or any other means, without written permission from the publisher.

John Benjamins Publishing Co. · P.O. Box 36224 · 1020 ME Amsterdam · The Netherlands John Benjamins North America · P.O. Box $27519 \cdot$ Philadelphia PA 19118-0519 • USA 


\section{Table of contents}

Introduction

The borrowers: Researching the cognitive aspects of translation

Sharon O'Brien

Cognitive load in simultaneous interpreting: Measures and methods

Kilian G. Seeber

Extended translation: A sociocognitive research agenda

Hanna Risku and Florian Windhager

Towards a new linguistic-cognitive orientation in translation studies

Juliane House

Translation competence: Explaining development and stagnation from a dynamic systems perspective

Susanne Göpferich

Applying a newswriting research approach to translation

Maureen Ehrensberger-Dow and Daniel Perrin

Metaphor in translation: Possibilities for process research

Christina Schäfner and Mark Shuttleworth

Investigating the conceptual-procedural distinction in the translation process: A relevance-theoretic analysis of micro and macro translation units

Fabio Alves and José Luiz Gonçalves

The role of archival and manuscript research in the investigation of translator decision-making

Jeremy Munday

Sound effects in translation

Inger M. Mees, Barbara Dragsted, Inge Gorm Hansen and Arnt Lykke Jakobsen

Subject index 



\section{Introduction}

This volume is devoted to Interdisciplinarity in Translation and Interpreting Process Research and was originally published as a special issue of Target $(25: 1,2013)$. It brings together a collection based on papers presented in 2011 at the Second International Research Workshop on Methodology in Translation Process Research held at Justus Liebig University, Giessen, Germany, and at the Research Models in Translation Studies II Conference, which took place at the University of Manchester, Great Britain.

In the first chapter, Sharon O'Brien gives an overview of the disciplines and sub-disciplines that cognitive translatology has borrowed from, specifying in what respects cognitive translatology has drawn on these, and makes suggestions for its future development, including ways in which it could turn into a lender exerting influence on other disciplines.

The chapter by Kilian Seeber discusses four of the most common methods of measuring the cognitive load generated by simultaneous interpreting tasks. He provides examples of the application of each method and highlights their respective advantages and disadvantages. The main focus of his contribution is on the psycho-physiological method of pupillometry, which he advocates as one of the most promising approaches.

Hanna Risku and Florian Windhager take account of the extended and situated nature of human cognition and, with the aim of investigating translation processes at a macro-level, combine current developments in cognitive science with a sociological perspective based on actor network theory and activity theory.

Juliane House pleads for a new linguistic-cognitive orientation in translation studies as a counterbalance to the current strong wave of socially- and culturally-oriented research around translation. After a review of studies employing verbal methods and behavioural experiments, she assesses the value of neurolinguistic studies for translation and, on this basis, suggests a combination of a translation theory and a neuro-functional theory of bilingualism as a starting point for gaining further insights into the cognitive reality of translation. 
Drawing on results from the TransComp research project on translation competence development, Susanne Göpferich seeks to explain the development and stagnation of translation competence from the point of view of Dynamic Systems Theory (DST), a theory that originated in mathematics and has found its way into various other disciplines, such as second-language acquisition and writing research, but, until now, has not been applied in translation studies. DST views competences as sets of variables that are interconnected and that interact over time.

Maureen Ehrensberger-Dow and Daniel Perrin illustrate how a mixedmethod approach that combines keystroke logging, screen recording, and cuebased retrospective verbalization and was originally developed to study the newswriting processes of journalists can be applied in translation process research to gain insights into the cognitive aspects of translation.

Christina Schäffner and Mark Shuttleworth explore the benefits of closer interaction between metaphor studies and translation process research using verbal data, keystroke logging and eye tracking to explore metaphor comprehension and translation. In this chapter, they also plead for the application of a multilingual approach (i.e., the translation of a source text into a range of different languages) to increase both the number and generality of examples available for analysis.

Fabio Alves and José Luiz Gonçalves argue that the conceptual-procedural distinction assumed in relevance theory can be useful in accounting for processing effort in translation. They use keystroke logging data to define micro translation units, which they then annotate and analyze in terms of the linguistic complexity and relative distance of editing procedures in related units. Their results suggest that considering processing effort from a relevance-theoretic perspective not only offers insights into translation but also contributes to validating some of relevance theory's claims.

Jeremy Munday approaches translator decision-making in the translation process from the perspective of archival and manuscript research, something more familiar to literary studies than translation studies. In this chapter, Munday demonstrates how archival material can illuminate questions on translation and revision processes and shows how it has resonance with the more empiricalexperimental paradigm as well as with the liberal arts paradigm. He concludes with interesting questions about the nature of 'archives' in the digital age.

In the last chapter, Inger M. Mees, Barbara Dragsted, Inge Gorm Hansen and Arnt Lykke Jakobsen report on the findings of a pilot study in which speech recognition software was employed for translation and in which this translation mode was compared with sight translation and written translation. The findings suggest that assignments which require a combination of knowledge from 
various disciplines, such as translation studies, interpreting studies, phonetics and phonology, can motivate students to learn more about different subjects because the interdisciplinary approach demonstrates to them what each discipline is able to contribute to their practical work.

Winterthur, Giessen and Dublin

January 2015

Maureen Ehrensberger-Dow, Susanne Göpferich and Sharon O’Brien 



\title{
The borrowers
}

\section{Researching the cognitive aspects of translation}

\author{
Sharon O'Brien \\ Dublin City University, Ireland
}

\begin{abstract}
This chapter considers the interdisciplinary interaction of research on the cognitive aspects of translation. Examples of influence from linguistics, psychology, neuroscience, cognitive science, reading and writing research and language technology are given, with examples from specific sub-disciplines within each one. The breadth of borrowing by researchers in cognitive translatology is made apparent, but the minimal influence of cognitive translatology on the respective disciplines themselves is also highlighted. Suggestions for future developments are made, including ways in which the domain of cognitive translatology might exert greater influence on other disciplines.
\end{abstract}

Keywords: translation process research, cognition, cognitive translatology, interdisciplinarity, reciprocity

\section{Introduction}

Translation process research has been ongoing for approximately thirty years now, but the field has grown significantly in the last decade or so, as evidenced by the number of recent publications dedicated to the topic (see, for example, the volumes by Hansen 1999; Alves 2003; Göpferich 2008; Göpferich et al. 2008; Mees et al. 2010; Shreve and Angelone 2010; O'Brien 2011a). The impetus for this growth, in my opinion, is due to a thirst for a greater understanding of translation as an expert task. The growth in research has also come about due to the development and increased accessibility of tools and methods for measuring specific cognitive aspects of the translation task, in particular screen recording, keystroke logging and eye-tracking technologies.

This development has not happened in a vacuum. The objectives of this chapter are to give a broad sketch of the disciplines and sub-disciplines from which research in cognitive translatology has borrowed, to examine the direction of 
influence and to reflect on the extent to which progress has been made in this research field and on how we might push the frontiers further. The term cognitive translatology is taken from Muñoz Martín (2010a) to cover research on the cognitive aspects of translation. Section 2 discusses the domains and sub-domains from which research in cognitive translatology has borrowed substantially in the last decade or so, mentioning specific examples for each one. Section 3 then reflects on the progress made, the direction of the influence and makes some suggestions on how the field might develop in the future.

\section{Borrowing from specific disciplines and sub-disciplines}

A broad sweep of the published research on cognitive translatology rapidly reveals that research has been influenced and inspired by a variety of disciplines, some of which are closely related to translation studies, others of which are more distant. Influence from disciplines such as linguistics, psychology, neuroscience, cognitive science, reading and writing research and language technology is clearly apparent. Within each of these disciplines, specific sub-disciplines have exercised particular influence. In what follows, the disciplines and sub-disciplines will be mentioned, along with the paradigms within those disciplines that have been drawn on by researchers, with examples given for each. The examples given here have been selected because they are good examples of interdisciplinary influences within translation process research.

The classification of disciplines and sub-disciplines is immediately problematic, of course. The main intention of the chapter is to illustrate the extent and nature of borrowing, rather than to create a rigorous typology of disciplines and sub-disciplines. Some flexibility with regards to the categorisation is therefore required.

\subsection{Linguistics}

Unsurprisingly, the very broad domain of linguistics is heavily drawn on by researchers of cognitive translatology. While cognitive translatology has as its main focus the process of translation, debate has also taken place on the importance of a parallel analysis of the translated product. The argument is that by looking only at the process or the product during a research project, one is looking at only one side of a coin. Hence, the sub-discipline of corpus linguistics has been drawn on to aid research in cognitive translatology. Corpora of translated texts allow the researcher to systematically describe the translated product, and, if so constructed, at different points in the translation process (e.g., prior to and following the (self-) revision stage). Alves et al. (2010), for example, expound the benefits of 
annotated corpora in identifying translation units associated with increased levels of cognitive effort during the translation process and Alves and Vale (2011) have developed a tool with which translation process data can be added to corpus data. While examples of research combining corpus linguistics with cognitive research are still few, there is no doubt much to be learned by combining the strengths of both approaches.

In cognitive translatology research, an important element in the research design is the nature of the source text selected for translation. Texts are selected for their appropriateness for the participants in a research project; for their level of specialism or, perhaps more commonly, for their level of generality; for their display of specific linguistic features (e.g., metaphor); or for their level of (perceived) difficulty. The selection of texts for a research project and the profiling of those texts are, however, often problematic because selection procedures have not been adequately operationalised. For example, it is tempting to use the rather old and inappropriate measures of readability indices (e.g., the Flesch Reading Ease, Flesch-Kincaid or Gunning Fog Index) as a measure of a source text's translation difficulty, but these measures have not shown themselves to correlate well with translation difficulty (O'Brien 2010). New and more reliable measures for text profiling are needed. For example, Alves, Pagano and da Silva (2010) employ Taboada and Mann's (2006) concept of Rhetorical Structure Theory as a method for profiling texts for research in cognitive translatology. This proposal has not yet seen much uptake in cognitive translatology research, but has scope for further investigation and testing.

Psycholinguistics, in particular the sub-discipline of bilingualism research, although removed in methodology from cognitive translatology, has also exerted some influence. In this case, however, it seems it is more a case of bilingualism researchers being enticed into the field of translation research than the opposite. Two recent examples of bilingualism research that also consider translation are Rydning and Lachaud (2010) and Lachaud (2011). In the former, the researchers examine the effect of context on polysemy during comprehension and production, comparing the performance of translators with bilinguals. Lachaud (2011) examines the process of transcoding deceptive, true and non-cognates in the bilingual brain and makes the first, albeit small, steps towards considering how transcoding might be used to help 'prompt' translators during the translation process.

\subsection{Psychology}

As research into the process of translation largely focuses on human translators and influences on their cognitive processes, strategies and behaviour, it is logical that the discipline of psychology has exerted some influence. Psychology is, of 
course, a very broad domain and the influences have come from two sub-domains in particular: expertise studies and, to a lesser extent, psychometrics.

Translation is regularly conceptualised as an 'expert task' requiring specific competences, all of which have to be strategically managed to reach a successful outcome (see, for example, PACTE 2003 and Göpferich 2009). The acquisition of such competences have been of special interest to translation process researchers, as have comparisons of 'experts' versus 'novices', such as student translators and bilinguals with no specific translator training (e.g., Jäskeläinen and TirkkonenCondit 1991; Jakobsen 2005). This research agenda has become even more important in recent years with the increase in crowd-sourcing and volunteer translation. Significant research has been carried out in general on the nature of expertise (e.g., Smith and Ericsson 1991; Dreyfus and Dreyfus 2005), its acquisition, and on the profiling of experts, and it is to this body of knowledge that translation process researchers have turned in order to understand translation as an expert task. As with text profiling, mentioned above, a particular challenge continues to be the profiling of participants in research projects along the cline of 'expertise. As a response, for example, Muñoz Martín (2010a) proposes the Wechsler Adult Intelligence Scale and TOEFL (Teaching of English as a Foreign Language) sub-tests as being useful for filtering out 'irregular' participants and for ranking 'regular' participants. In another article (2010b), the same author discusses the concept of expertise in general and how it can contribute to the field of cognitive translatology. Jääskeläinen (2010) tackles the concept of 'professional translator', a concept used frequently in cognitive translatology, and asks the difficult question "Are all professionals experts?", giving consideration to the definition of an expert and arguing for the reinterpretation of research evidence in process studies on the basis of these definitions.

A related topic, but one that has received little attention to date, is the relationship between personality and the translation process. Does personality type have any effect on the translation process, on strategies used (e.g., risk-averse versus risk-taking strategies), and even on the product (e.g., level of creativity in a translation)? There are many intriguing questions that could be posed, but that have not yet been investigated. Interestingly, the domain of psychometrics is the focus of some attention now in cognitive translatology. An example of early-stage research on this topic is Hubscher-Davidson's (2009) preliminary study of psychometric profiling and potential correlations with translation quality.

Of interest to researchers in cognitive translatology is what happens in the brain during the process of translation. The preferred method for gaining indirect information about what translators do during the translation process has been verbal protocols (Ericsson and Simon 1993), delivered either concurrently with the translation task or retrospectively, with screen recording and keystroke 
logging frequently used as recall aids. The use of verbal protocols as a means of gaining access to brain activity is strongly connected to research into expertise, which was mentioned previously. Much consideration has been given to the use of verbal reports as a method for understanding cognitive processing, both outside and within the domain of translation studies, and the advantages and disadvantages of this method are well recognised (see, for example, Lörscher 1988; Krings 1986). In particular, the automatisation of expertise, that is when there is no verbalisable awareness of strategies or processes, has been recognised as a drawback, as has the fact that the production of concurrent protocols slows a task down by approximately 30\% (Krings 2001) and shortens translation units (Jakobsen 2003).

\subsection{Neuroscience}

The limitations of verbal reports as well as the increasing ease of access to technologies such as eye tracking (the recording of eye gaze data on an area of interest, e.g., a computer monitor and text, during a task), EEG (electroencephalography, i.e., the recording of electrical activity on the scalp) and fMRI (functional Magnetic Resonance Imaging, i.e., the measurement of changes in blood flow in the brain as a result of brain activity) have resulted in new ground being broken in cognitive translatology. Eye tracking will be discussed in Section 2.4, but here two translation-related studies that have discussed or actually used fMRI scanners in a bid to understand what might occur in the brain during translation and interpreting can be mentioned. Chang (2009) uses both an fMRI scanner and an eye tracker to investigate cognitive load in directionality (translating from L1 to L2 and vice versa). This was an early-stage study which recorded fMRI measures while participants silently translated. Moser-Mercer (2010) draws on the domain of neuroscience in her discussion of the plasticity of the brain, the role of long-term and short-term memory and of deliberate practice in the acquisition of interpreting expertise.

\subsection{Cognitive science}

In studying cognition in translation, it is not surprising that cognitive science has been influential, with the volume by Danks and Shreve paving the way in 1997. Metacognition, or cognition about cognition, has been of particular interest in the study of translation strategies and competences. Angelone (2010) and Angelone and Shreve (2011) are two recent studies which draw on the field of cognition to increase understanding about how translators manage uncertainty and solve problems during the process of translation, and what effect their ability to do so has on the quality of the translated product. 
Long-term memory (LTM), short-term memory (STM), memory capacity and the role they play in product quality have been of particular interest in the field of interpreting for a long period (see, for example, Gambier et al. 1994). Baddeley and Hitch's (1974) model of working memory has been particularly influential. Translation process research has also been interested in these concepts, especially when considering the impact of automatisation on the usefulness of verbal protocols, where STM is said to play a role. Some work has also been done on comparing sight translation and interpreting for demands on working memory capacity (e.g., Agrifoglio 2004).

A third area of influence from the domain of cognitive science is the study of eye-mind coordination, with Just and Carpenter's (1980) eye-mind hypothesis being drawn on heavily as a paradigm for eye-tracking analyses of translation processes. The eye-mind hypothesis states that there is no appreciable lag in time between what the eye fixates on and what is processed in the brain; it is therefore useful for the analysis of attention (on source text, target text, terminology or other resources) during the translation process. Eye tracking, along with screen recording and keystroke logging, has also opened up the possibility of measuring cognitive load in the translation task, with number and duration of pauses, number of revisions, number and duration of fixations and changes in pupil dilations all being used as measures of cognitive load, sometimes accompanied by verbal protocols for triangulation purposes. Cognitive load in translation has, for example, been measured by O'Brien (2006; 2008), specifically in the context of translation memory (TM) tools, with comparisons being made between different TM match types, ranging from exact matches (suggesting that no revisions are required) to fuzzy and machine-translation generated matches (suggesting that some revision is required).

Although also linked to the domain of sociology, situated, embodied cognition can be mentioned here as a paradigm of cognitive science which has influenced cognitive translatology, given its focus on human cognition and how it is used to interpret and take account of what is going on around us. Situated, embodied cognition is not only interested in what goes on in the human brain, but broadens the scope to examine the whole human being, their history and environment (Risku 2010). This holistic approach allows researchers in cognitive translatology to examine not only what translators do during the translation process, but also how environmental and social factors influence their decision-making as experts in their fields. Risku (2010) argues in favour of the use of the situated, embodied cognition framework to help develop research in cognitive translatology and in technical communication. 


\subsection{Writing and reading}

Much research has been conducted in the domain of monolingual information processing, notably around the tasks of writing, reading and revising. Cognitive translatology has looked to research in these sub-domains to help understand those components of the translation process that involve reading and writing.

The eye-mind hypothesis, mentioned earlier, has been employed to a significant extent in reading studies, which have made extensive use of eye tracking as a research methodology. The uptake of eye tracking in cognitive translatology has enabled the study of reading (of both source and target text) during the translation process. Moreover, monolingual studies of the readability of texts have inspired translation researchers to test readability indicators for their relevance to translation research (e.g., Jensen 2009 and O'Brien 2010), and to even use readability as a measure of quality for automatically translated text (Doherty et al. 2010).

Surprisingly, although one of the most important components of translation is writing, research into monolingual writing and revision processes has had a lower influence on cognitive translatology than one might expect. The two fields have much in common with their interests in metacognition, strategy, expertise, pause analysis and writer's profiles (cf. Van Waes and Schellens 2003, for example) as well as in keystroke logging, screen recording and eye tracking as methods. Nonetheless, there is some evidence now of influence of one domain on the other. For example, the writing process researchers Schrijver et al. (2011) recently investigated the concept of 'transediting', i.e., the manipulation of the source text content and structure within the target text in order to adhere to target text genre specifications in a study on patient information leaflets. Also, there is evidence of collaboration between translation process researchers and researchers into writing processes in the field of journalism (Ehrensberger-Dow and Perrin 2010).

\subsection{Language technology}

The increasing technologisation of the translation profession and, along with it, the translation process, has been noted elsewhere (e.g., Austermühl 2001; Bowker 2002; Quah 2006). The impact tools have on the translated product and process, and on the working lives of translators, has been a particular area of focus in recent years. Additionally, the increasing use of automatic or machine translation has necessitated that translation scholars turn their attention to that specialised field.

A focus on the cognitive load of processing different types of matches from translation memory tools has already been alluded to above. More generally, translation process research has started to ask questions about the usability and suitability of these tools for the translation process. One small study looked at the 
'concordance feature' in a translation memory interface using eye tracking as an instrument of measurement (O’Brien et al. 2010). Drawing on the domain of human-computer interaction, another study investigated the topic of machine translation in the translator's workplace and noted how translators often see machine translation as a black box, which removes them from the task of translation and diminishes its collaborative nature (Karamanis et al. 2011). A third study looked more generally at translators' interaction with technology in the workplace, using the method of contextual inquiry commonly used in studies of human-computer interaction (Désilets et al. 2008).

Machine translation, by its nature, draws on computational linguistics. Translation process research would seem far removed from this domain, but recent attempts again seek to draw connections by investigating correlations between the far-removed computational, automatic algorithms for the measurement of machine translation quality (see, for example, Papineni et al. 2002; CallisonBurch et al. 2008; Lavie and Przybocki 2009), on the one hand, and the cognitive effort of the post-editing process on the other (O’Brien 2011b).

\section{Progress and direction}

The review in Section 2 demonstrates the breadth of influence from other domains on research in cognitive translatology. Commencing with an interest in memory capacity and moving to studies of expertise, cognition, text and translator profiling, to translator and technology interaction, the domain has evolved and grown significantly in recent years. Moreover, the tools and methods employed have increased in range and complexity.

Strikingly, the direction of influence seems to be largely one-way, that is, translation scholars appear to borrow liberally from domains such as linguistics, cognitive science, neuroscience and so on, but the range of influence from translation studies on those domains and sub-domains appears to be very limited at the present time. Choi and Pak (2006) characterise interdisciplinarity as 'working between' two or more disciplines, but they also characterise it as having a level of 'reciprocity'. There is ample evidence that researchers in cognitive translatology are 'working between' disciplines, but, as yet, there is little evidence of reciprocity.

There is little doubt that the domain of cognitive translatology has matured over the last few years, but it is arguably still in its infancy. There are many ways in which further development could take place by borrowing even more from more established disciplines. For example, techniques used in the domain of forensic linguistics to measure author attribution and homogeneity between texts in corpora (see, for example, Vogel and Lynch 2008) could feasibly be employed in translation 
process research to establish similarities across source texts used in experiments. The use of Rhetorical Structure Theory, or something similar (see, for example, the Código research project), ${ }^{1}$ could be investigated in more detail. Researchers could draw more substantially from the field of expertise studies by, for example, seeking to test Dreyfus and Dreyfus' (2005) proposed 5-stage model of expertise (ranging from Novice, to Advanced Beginner, Competence, Proficiency and finally to Expertise) on translators. More research could be done on the field of psychometrics and we could further utilise ethnographic and HCI (human-computer interaction) methods to understand more about translation and technology. These are but a handful of suggestions.

Consolidation in the research domain of cognitive translatology is likely to lead eventually to influences on other domains. Consolidation can be achieved by building on the already interdisciplinary nature of the domain, by collaborating more with researchers within the domain, by sharing tools, expertise, data (see, for example, Göpferich 2010) and by inviting researchers from other domains to collaborate. Gradually, we will move towards reciprocal interdisciplinarity (Göpferich 2011), in which TS is not only a borrower but also a lender. Such a development would offer a number of potential benefits, enabling us to provide different perspectives on complex problems, to increase creativity and avoid a situation where individual disciplines become tired and predictable (Nissani 1997).

\section{References}

Agrifoglio, Marjorie. 2004. "Sight Translation and Interpreting: A Comparative Analysis of Constraints and Failures." Interpreting - International Journal of Research and Practice in Interpreting 6 (1): 43-68. DOI: 10.1075/intp.6.1.05agr

Alves, Fabio (ed). 2003. Triangulating Translation. Amsterdam: John Benjamins. DOI: 10.1075/ btl.45

Alves, Fabio, Adriana Pagano, Stella Neumann, Erich Steiner, and Silvia Hansen-Schirra. 2010. "Translation Units and Grammatical Shifts: Towards an Integration of Product- and Process-Based Translation Research.” In Translation and Cognition, ed. by Gregory Shreve, and Erik Angelone, 109-142. Amsterdam: John Benjamins. DOI: 10.1075/ata.xv.07alv

Alves, Fabio, Adriana Pagano, and Igor da Silva. 2010. "A New Window on Translators Cognitive Activity: Methodological Issues in the Combined Use of Eye Tracking, Key Logging and Retrospective Protocols." In Methodology, Technology and Innovation in Translation Process Research [Copenhagen Studies in Language, 38], ed. by Inger Mees, Fabio Alves, and Susanne Göpferich, 267-292. Copenhagen: Samfundslitteratur.

1. http://www.cogtrans.net/codigoEN.htm (last accessed 24/01/2015) 
Alves, Fabio, and Daniel Vale. 2011. "On Drafting and Revision in Translation: A Corpus Linguistics Oriented Analysis of Translation Process Data." TC3: Translation: Computation, Corpora, Cognition 1 (1): 105-122.

Angelone, Erik. 2010. "Uncertainty Management and Metacognitive Problem Solving." In Translation and Cognition, ed. by Gregory Shreve, and Erik Angelone, 17-40. Amsterdam: John Benjamins. DOI: 10.1075/ata.xv.03ang

Angelone, Erik, and Gregory Shreve. 2011. "Uncertainty Management, Metacognitive Bundling in Problem Solving, and Translation Quality." In Cognitive Explorations of Translation, ed. by Sharon O'Brien, 108-130. London: Continuum.

Austermühl, Frank. 2001. Electronic Tools for Translators. Manchester: St. Jerome.

Baddeley, Alan D., and Graham Hitch. 1974. "Working Memory." In The Psychology of Learning and Motivation: Advances in Research and Theory, Vol. 8, ed. by Gordon H. Bower, 47-89. New York: Academic Press.

Bowker, Lynne. 2002. Computer-Aided Translation Technology: A Practical Introduction. Ottawa: University of Ottawa Press.

Callison-Burch, Chris, Cameron Fordyce, Philipp Koehn, Christof Monz, and Josh Schroeder. 2008. "Further Meta-Evaluation of Machine Translation." Proceedings of ACL-08: HLT. Third Workshop on Statistical Machine Translation, 70-106. The Ohio State University, Columbus, Ohio, June 19. DOI: 10.3115/1626394.1626403

Chang, Vincent Chieh-Ying. 2009. Testing Applicability of Eye-Tracking and fMRI to Translation and Interpreting Studies: An Investigation into Directionality. Unpublished Ph.D. dissertation, Imperial College London.

Choi, Bernard, and Anita Pak. 2006. "Multidisciplinarity, Interdisciplinarity and Transdisciplinarity in Health Research, Services, Education and Policy: 1. Definitions, Objectives, and Evidence of Effectiveness." Clinical and Investigative Medicine 29 (6): 351364.

Danks, Joseph, and Gregory Shreve. 1997. Cognitive Processes in Translation and Interpreting. Thousand Oaks, CA: Sage Publications.

Désilets, Alain, Louise Brunette, Christiane Melançon, and Geneviève Patenaude. 2008. "Reliable Innovation - A Tecchie's Travels in the Land of Translators." 8th Association of Machine Translation in the Americas Conference, 339-345. Hawaii, 21-25 October.

Doherty, Stephen, Sharon O’Brien, and Michael Carl. 2010. “Eye Tracking as an MT Evaluation Technique." Machine Translation 24 (1): 1-13. DOI: 10.1007/s10590-010-9070-9

Dreyfus, Hubert, and Stuart Dreyfus. 2005. "Peripheral Vision: Expertise in Real World Contexts.” Organization Studies 26 (5): 779-792. DOI: 10.1177/0170840605053102

Ehrensberger-Dow, Maureen, and Daniel Perrin. 2010. "Progression Analysis. Tracing Journalistic Language Awareness." 5th International Conference on ELT in China, 485-510. Beijing: Foreign Language Teaching and Research Press.

Ericsson, K. Anders, and Herbert Simon. 1993. Protocol Analysis: Verbal Reports as Data. Cambridge, MA: MIT Press.

Gambier, Yves, Daniel Gile, and Christopher Taylor. 1994. Conference Interpreting: Current Trends in Research, Proceedings of the International Conference on Interpreting - What do We Know and How? Turku, August 25-27. Amsterdam: John Benjamins. DOI: 10.1075/ btl.23.01gil

Göpferich, Susanne. 2008. Translationsprozessforschung. Stand - Methoden - Perspektiven. Tübingen: Narr. 
Göpferich, Susanne. 2009. “Towards a Model of Translation Competence and its Acquisition: The Longitudinal Study TransComp." In Behind the Mind: Methods, Models and Results in Translation Process Research [Copenhagen Studies in Language, 37], ed. by Susanne Göpferich, Arnt Jakobsen, and Inger Mees, 11-37. Copenhagen: Samfundslitteratur.

Göpferich, Susanne. 2010. "Data Documentation and Data Accessibility in Translation Process Research.” The Translator 16 (1): 93-124. DOI: 10.1080/13556509.2010.10799295

Göpferich, Susanne. 2011. "From Multidisciplinarity to Transdisciplinarity: The Investigation of Competence Development as a Case in Point." MikaEL Electronic Proceedings of the KäTu Symposium on Translation and Interpreting Studies 5: 1-24.

Göpferich, Susanne, Arnt Lykke Jakobsen, and Inger Mees (eds). 2008. Looking at Eyes. Eye Tracking Studies of Reading and Translation Processing [Copenhagen Studies in Language, 36]. Copenhagen: Samfundslitteratur.

Hansen, Gyde (ed). 1999. Probing the Process in Translation - Methods and Results [Copenhagen Studies in Language, 24]. Copenhagen: Samfundslitteratur.

Hubscher-Davidson, Severine. 2009. "Personal Diversity and Diverse Personalities in Translation: A Study of Individual Differences." Perspectives: Studies in Translatology 17 (3): 175-192. DOI: 10.1080/09076760903249380

Jääskeläinen, Riitta. 2010. "Are All Professionals Experts? Definitions of Expertise and Reinterpretation of Research Evidence in Process Studies." In Translation and Cognition, ed. by Gregory Shreve, and Erik Angelone, 213-227. Amsterdam: John Benjamins. DOI: 10.1075/ata.xv.12jaa

Jääskeläinen, Riitta, and Sonja Tirkkonen-Condit. 1991. "Automatised Processes in Professional vs. Non-Professional Translation: A Think-Aloud Protocol Study." In Empirical Research in Translation and Intercultural Studies, ed. by Sonja Tirkkonen-Condit, 89-109. Tübingen: Gunter Narr.

Jakobsen, Arnt Lykke. 2003. "Effects of Think Aloud on Translation Speed, Revision and Segmentation." In Triangulating Translation: Perspectives in Process-Oriented Research, ed. by Fabio Alves, 69-95. Amsterdam: John Benjamins. DOI: 10.1075/btl.45.08jak

Jakobsen, Arnt Lykke. 2005. "Investigating Expert Translators' Processing Knowledge." In Knowledge Systems and Translation - Text, Translation and Computational Processing 7 , ed. by Helle van Dam, Jan Engberg, and Heidrun Gerzymisch-Arbogast, 173-189. Berlin: Mouton de Gruyter.

Jensen, Kristian T.H. 2009. "Indicators of Text Complexity." In Behind the Mind: Methods, Models and Results in Translation Process Research [Copenhagen Studies in Language, 37], ed. by Susanne Göpferich, Arnt Jakobsen, and Inger Mees, 61-80. Copenhagen: Samfundslitteratur.

Just, Marcel A., and Patricia Carpenter. 1980. "A Theory of Reading: From Eye Fixation to Comprehension.” Psychological Review 87: 329-354. DOI: 10.1037/0033-295X.87.4.329

Karamanis, Nikiforas, Saturnino Luz, and Gavin Doherty. 2011. "Translation Practice in the Workplace: A Contextual Analysis and Implications for Machine Translation.” Machine Translation 25 (1): 35-52. DOI: 10.1007/s10590-011-9093-x

Krings, Hans P. 1986. Was in den Köpfen von Übersetzern vorgeht. Tübingen: Narr.

Krings, Hans P. 2001. Repairing Texts: Empirical Investigations of Machine Translation PostEditing Processes. Translated by G. S. Koby. Kent, OH: The Kent State University Press.

Lachaud, Christian M. 2011. "EEG, EYE and Key: Three Simultaneous Streams of Data for Investigating the Cognitive Mechanisms of Translation." In Cognitive Explorations of Translation, ed. by Sharon O’Brien, 131-153. London: Continuum. 
Lavie, Alon, and Mark A. Przybocki (eds). 2009. "Automated Metrics for Machine Translation Evaluation.” Special Issue of Machine Translation 23 (2/3): 69-70. DOI: 10.1007/s10590010-9071-8

Lörscher, Wolfgang. 1988. "Thinking-Aloud as a Method for Collecting Data on Translation Processes.” In Empirical Research in Translation and Intercultural Studies, ed. by Sonja Tirkkonen-Condit, 67-77. Tübingen: Gunter Narr.

Mees, Inger, Fabio Alves, and Susanne Göpferich (eds). 2010. Methodology, Technology and Innovation in Translation Process Research [Copenhagen Studies in Language, 38]. Copenhagen: Samfundslitteratur.

Moser-Mercer, Barbara. 2010. “The Search for Neuro-Physiological Correlates of Expertise in Interpreting." In Translation and Cognition, ed. by Gregory Shreve, and Erik Angelone, 263-288. Amsterdam: John Benjamins. DOI: 10.1075/ata.xv.15mos

Muñoz Martín, Ricardo. 2010a. "The Way they Were: Subject Profiling in Translation Process Research.” In Methodology, Technology and Innovation in Translation Process Research [Copenhagen Studies in Language, 38], ed. by Inger Mees, Fabio Alves, and Susanne Göpferich, 87-108. Copenhagen: Samfundslitteratur.

Muñoz Martín, Ricardo. 2010b. “On Paradigms and Cognitive Translatology.” In Translation and Cognition, ed. by Gregory Shreve, and Erik Angelone, 169-187. Amsterdam: John Benjamins. DOI: 10.1075/ata.xv.10mun

Nissani, Moti. 1997. "Ten Cheers for Interdisciplinarity: The Case for Interdisciplinary Knowledge and Research." The Social Science Journal 34 (2): 201-216. DOI: 10.1016/S03623319(97)90051-3

O’Brien, Sharon. 2006. “Eye Tracking and Translation Memory Matches." Perspectives: Studies in Translatology 14 (3): 185-205.

O’Brien, Sharon. 2008. "Processing Fuzzy Matches in Translation Memory Tools: An EyeTracking Analysis." In Looking at Eyes. Eye Tracking Studies of Reading and Translation Processing [Copenhagen Studies in Language, 36], ed. by Susanne Göpferich, Arnt Lykke Jakobsen, and Inger Mees, 79-102. Copenhagen: Samfundslitteratur.

O’Brien, Sharon. 2010. "Controlled Language and Readability." In Translation and Cognition, ed. by Gregory Shreve, and Erik Angelone, 143-168. Amsterdam: John Benjamins. DOI: 10.1075/ata.xv.08obr

O’Brien, Sharon (ed). 2011a. Cognitive Explorations of Translation. IATIS Yearbook 2010. London: Continuum.

O’Brien, Sharon. 2011b. “Towards Predicting Post-Editing Productivity." Machine Translation 25 (3): 197-215. DOI: 10.1007/s10590-011-9096-7

O’Brien, Sharon, Minako O’Hagan, and Marian Flanagan. 2010. “Keeping an Eye on the UI Design of Translation Memory: How do Translators Use the Concordance Feature?" In Proceedings of the 28th European Conference on Cognitive Ergonomics, ed. by WillemPaul Brinckman, and Mark Neerincx, 187-190. Delft University of Technology. DOI: $10.1145 / 1962300.1962338$

PACTE. 2003. “Building a Translation Competence Model.” In Triangulating Translation, ed. by Fabio Alves, 43-66. Amsterdam: John Benjamins. DOI: 10.1075/btl.45.06pac

Papineni, Kishore, Salim Roukos, Todd Ward, and Wei-Jing Zhu. 2002. "BLEU: A Method for Automatic Evaluation of Machine Translation.” In Proceedings of ACL-2002: 40th Annual Meeting of the Association for Computational Linguistics, 311-318. Philadelphia.

Quah, Chiew Kin. 2006. Translation and Technology. New York: Palgrave Macmillan. DOI: $10.1057 / 9780230287105$ 
Risku, Hanna. 2010. “A Cognitive Scientific View on Technical Communication and Translation: Do Embodiment and Situatednesss Really Make a Difference?” Target 22 (1): 94-111. DOI: 10.1075/target.22.1.06ris

Rydning, Antin Fougner, and Christian Michel Lachaud. 2010. “The Reformulation Challenge in Translation." In Translation and Cognition, ed. by Gregory Shreve, and Erik Angelone, 85-108. Amsterdam: John Benjamins. DOI: 10.1075/ata.xv.06ryd

Schrijver, Iris, Leona Van Vaerenbergh, and Luuk Van Waes. 2011. Transediting in Students' Translation Processes. Antwerp: Artesis Working Papers in Translation Studies 2010-3.

Shreve, Gregory, and Erik Angelone. 2010. Translation and Cognition. Amsterdam: John Benjamins. DOI: 10.1075/ata.xv.01shr

Smith, Jacqui, and K. Anders Ericsson. 1991. Towards a General Theory of Expertise: Prospects and Limits. Cambridge: Cambridge University Press.

Taboada, Maite, and William Mann. 2006. "Rhetorical Structure Theory: Looking Back and Moving Ahead.” Discourse Studies 8 (3): 423-459. DOI: 10.1177/1461445606061881

Van Waes, Luuk, and Peter Jan Schellens. 2003. "Writing Profiles: The Effect of the Writing Mode on Pausing and Revision Patterns of Experienced Writers." Journal of Pragmatics 35: 829-853. DOI: 10.1016/S0378-2166(02)00121-2

Vogel, Carl, and Gerard Lynch. 2008. “Computational Stylometry: Who's in Play?” In Verbal and Nonverbal Features of Human-Human and Human-Machine Interaction [Lecture Notes in Computer Science, Vol. 5042/2008], ed. by Anna Esposito, Nicolaos Bourbakis, Nikolaos Avouris, and Ioannis Hatzilygeroudis, 169-186. Berlin: Springer. DOI: 10.1007/978-3-54070872-8_13 



\title{
Cognitive load in simultaneous interpreting Measures and methods
}

\author{
Kilian G. Seeber \\ University of Geneva, Switzerland
}

The mental effort required to perform a simultaneous interpreting task or the cognitive load generated by it has attracted the interest of many a researcher in the field. To date, however, there is little agreement on the most suitable method to measure this phenomenon. In this contribution, I set out to discuss four of the most common methods of measuring cognitive load and the way in which they have been applied in interpreting research, providing examples for each and highlighting their respective advantages and disadvantages. The main focus of the contribution will be on pupillometry, a psycho-physiological method I deem to be among the most promising approaches to objectively measure cognitive load during simultaneous interpreting in real time.

Keywords: simultaneous interpreting, cognitive load, mental effort, analytical methods, subjective methods, performance methods, psycho-physiological methods, methodology, pupillometry

\section{Introduction}

Among interpreting scholars, the list of those who consider simultaneous interpreting a cognitively tasking activity (e.g., Gile 1995; Hyönä et al. 1995; Massaro and Shlesinger 1997; Moser-Mercer 1997; de Groot 2000) seems to extend beyond that of those who consider such statements as nothing but "primitives or clichés" (Setton 2003, 37). Indeed, Setton argues that concurrent sub-tasks during simultaneous interpreting can be performed "comfortably if they are all sharing the same representation" $(2001,5)$. Over the years, the notion of cognitive load generated by the interpreting task, or the amount of cognitive effort necessary to perform it, has generated a substantial amount of interest and has been addressed by scholars from within and outside the paradigm who believe that such investigation might be very fruitful (de Groot 1997). The amount of empirical evidence gathered to 
corroborate theories and claims about the amount of cognitive load generated by the task, however, would appear to be inversely proportional to the strength of the assertions put forward. As the following discussion will show, this imbalance may partially be explained by the difficulty of finding an appropriate paradigm within which to test hypotheses, coupled with a methodology capable of identifying, isolating and measuring the phenomenon as directly as possible. The purpose of this chapter is to provide an analysis of the potential and limitations of some of the most widely used methods for investigating cognitive load in simultaneous interpreting and the metrics (or measures) they employ, with a special focus on pupillometry.

\section{Measuring cognitive load}

The multidimensional nature of cognitive load makes it difficult to define. ${ }^{1}$ On the one hand, this construct represents the load imposed on the performer by a particular task (Paas and Merrienboer 1993), on the other hand, it represents the perceived effort invested by a performer during the execution of that task (Yin et al. 2008). For the purpose of the present analysis, cognitive load will be defined as the amount of capacity the performance of a cognitive task occupies in an inherently capacity-limited system (Miller 1956). Paas et al. (2003) and Schultheis and Jameson (2004) describe four discrete categories of methods for the assessment of cognitive load, all with their respective advantages and disadvantages: analytical methods, subjective methods, performance methods and psycho-physiological methods.

Analytical methods attempt to estimate cognitive load relying on subjective data, often elicited through expert opinion, and analytical data, often generated with mathematical models or task analysis (Paas et al. 2003). The advantage of this approach is that the cross-tabulation of subjective and analytical data can take place at a purely theoretical level thus avoiding sometimes cumbersome empirical testing; its major shortcoming is that it relies exclusively on prior knowledge both about the task and about the subjects and is therefore unable to take into account individual performance differences.

Subjective methods use self-reported data as a means to quantify phenomena that are perceived as difficult or impossible to assess objectively, such as cognitive load. Data are generated using introspection, as well as retrospective and concurrent verbalization, and are reflected in metrics such as rating scales. The advantage of these methods over analytical methods is that they involve task performers,

1. See Seeber (2011) for a more comprehensive discussion on the definition of cognitive load and how it relates to simultaneous interpreting. 
who have been shown to be able to provide an appreciation of their perceived mental load (Paas et al. 2003). Their drawback is a possible contamination of data by memory and consciousness effects, seeing that the response is usually timedelayed. With the exception of concurrent verbalization (i.e., think-aloud), selfrating and assessment takes place after the performance of the task.

Performance methods usually involve the simultaneous performance of a primary task and a secondary task with the goal of identifying the extent to which the latter affects the former (Haapalainen et al. 2010). The advantage of these methods is that they allow tasks to be studied without the need to de-compose them. Their major drawback is that uncontrolled processes might confound the causal relationship between the two tasks at hand.

Psycho-physiological methods, finally, assess the physiological processes known to co-vary with changes in cognitive load. In doing so, they provide a more direct and, given that these physiological responses are not subject to voluntary control, more objective measure of cognitive load. The drawback of these methods is their complexity; they can be invasive (to varying degrees) and therefore might interfere with the task itself.

What follows is an attempt to illustrate, by means of a few examples, how these methods have been used to qualify and quantify cognitive load phenomena in simultaneous interpreting.

\subsection{Cognitive load in interpreting research: Analytical methods}

Among the best-known analytical approaches to conceiving of cognitive load in interpreting are Gile's effort models (1995). The underlying framework of this "conceptual framework" (Gile, 2008,62) is similar to Kahneman's (1973) single resource theory that postulates the existence of a single pool of finite processing capacity to fuel all cognitive tasks. Summarizing more complex operations under the heading of four efforts, i.e., listening and analysis, production, memory, and coordination, Gile proposes a simple architecture to describe the amount of effort invested in the simultaneous performance of these operations to get from the input "I" to the total amount of invested effort "T" (see Figure 1). The appeal of this conceptual framework is its striking simplicity. Reducing the complex simultaneous interpreting task to an uncomplicated mathematical formula (which, as the author points out, should not be understood as a simple arithmetic sum) is a noteworthy feat. One of the examples Gile provides illustrates the processing capacity demands involved in the simultaneous interpretation of the sentence given at the bottom of Figure 1. The schematic convincingly illustrates how a local increase in information density (i.e., the section in italics) causes a knock-on effect eventually leading to load being exported to a subsequent processing stage. 
However, this model's main strength, i.e., its ability to capture the complex task of simultaneous interpreting and provide a simple and concise account of its intrinsic cognitive demands, constitutes a potential weakness for its application beyond the realm of teaching. It is possible that some of the parallel cognitive processes recruit the same resources. It is also possible, however, that some tasks involved in an online bilingual language processing task such as simultaneous interpreting are constrained by limitations going beyond those inherent to the three principal component tasks. Such limitations might only come to bear when the component tasks are combined into a more complex one. In other words, in terms of cognitive load, simultaneous interpreting might be more or less than the sum of its parts. It is conceivable that this incongruity cannot merely be accounted for by the coordination effort, which is assumed to be ever-present and thus not specifically represented in Gile's model (see Figure 1).

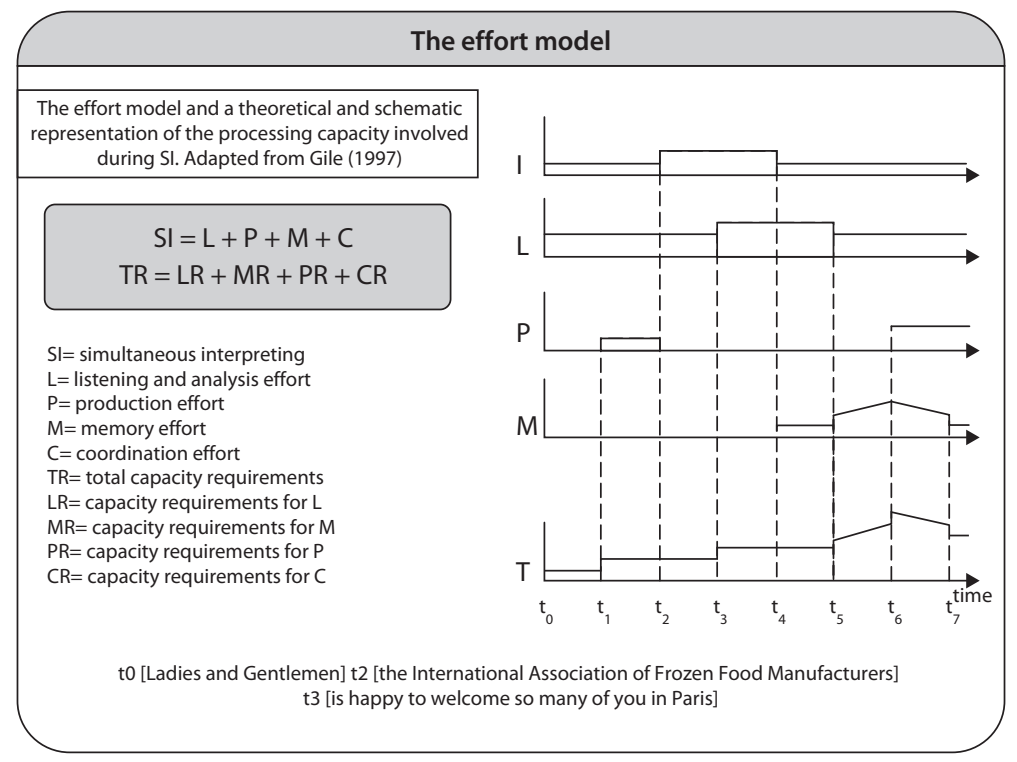

Figure 1. Adaptation of Gile's (1997) effort model

What is more, empirical findings do not lend unequivocal support to Kahneman's (1973) single resource theory. On the contrary, they suggest that structurally similar tasks interfere more with each other than structurally dissimilar ones do (Wickens 2002). I attempted to address some of these limitations by introducing the Cognitive Load Model as a competing account to the Effort Model (Seeber 2011). The former is based on Wickens' (1984; 2002) Multiple Resource Theory and assumes a finite amount of task-specific processing capacity as well as 
a certain amount of task-interference depending on the structural proximity of the tasks involved ${ }^{2}$ (see Figure 2).

The strength of the Cognitive Load Model is its ability to illustrate cognitive load taking into account both the input and output - something the Effort Model seems to fall short of. As the maximum local load ${ }^{3}$ is intrinsically linked to the amount of parallel processing (and thus interference generated by different tasks), as well as the amount of time for which elements need to be stored, the predictions

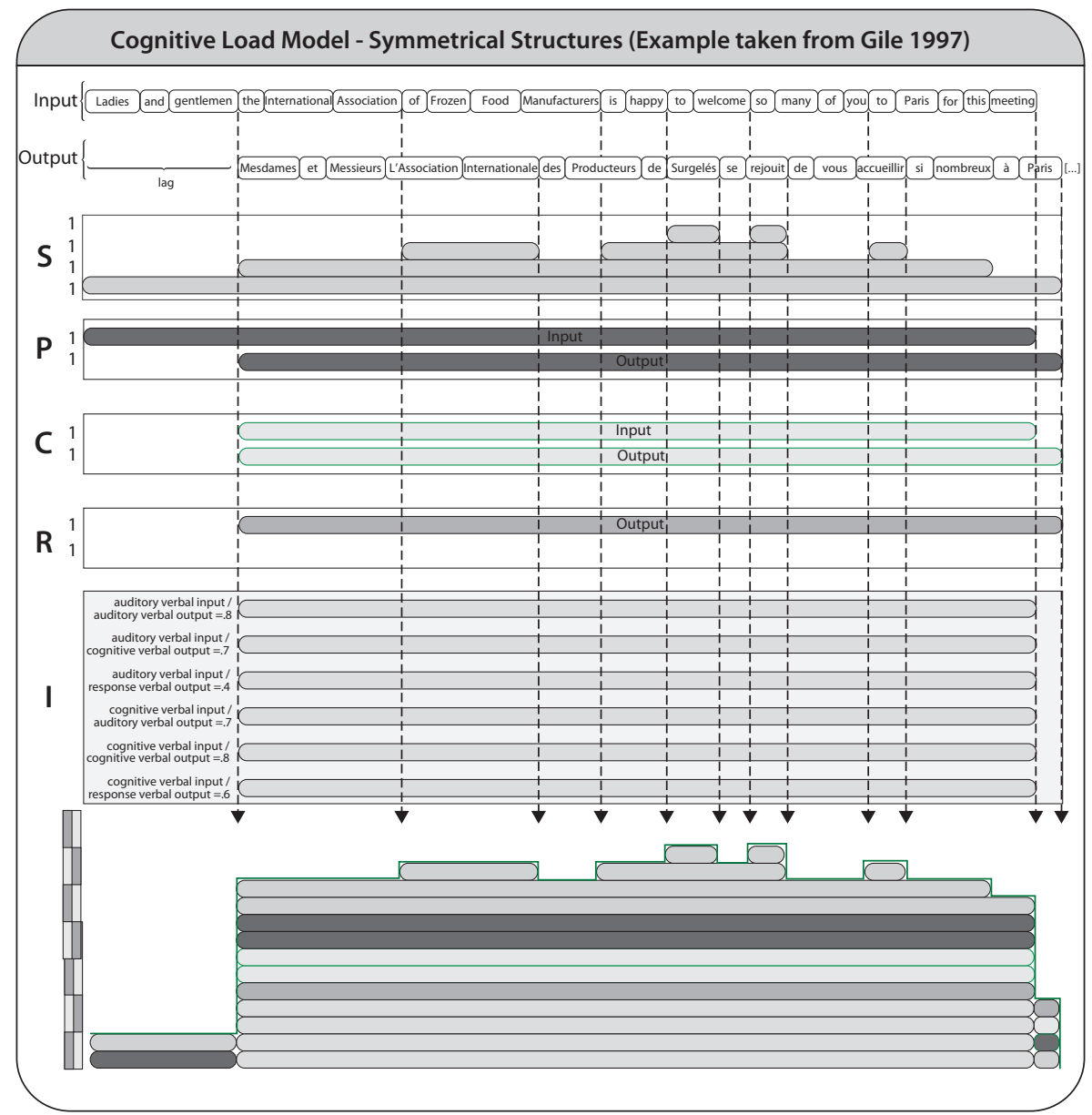

Figure 2. Seeber's (2011) Cognitive Load Model for symmetrical structures ( $\mathrm{S}=$ storage, $\mathrm{P}=$ perceptual auditory verbal processing, $\mathrm{C}=$ cognitive verbal processing, $\mathrm{R}=$ verbal response processing, $\mathrm{I}=$ interference)

2. For a more detailed description, see Wickens $(1984 ; 2002)$ as well as Seeber $(2007 ; 2011)$.

3. The maximum amount of load generated in a particular period of interest. 
of Gile's Effort Model appear somewhat arbitrary. The Cognitive Load Model for the same sentence, for instance, illustrates one (of several) ways of interpreting the sentence that keeps cognitive load fairly constant, without presupposing any particular background knowledge by the interpreter ${ }^{4}$ (the latter limitation is shared with Gile's Effort Model and is due to the nature of the measure used). These two examples of analytical measures of cognitive load illustrate the constraints inherent to an approach unable to take into account individual differences.

\subsection{Cognitive load in interpreting research: Subjective methods}

A review of the recent literature suggests that unlike analytical methods, subjective methods do not seem to be widely used to assess cognitive load in interpreting research. This might be attributable to two reasons. On the one hand, there appears to be little agreement on the reliability of self-reports of cognitive load. While some (e.g., Gopher and Braune 1984; Paas et al. 2003) found them to be relatively reliable, others (e.g., Mital and Govindaraju 1999) found evidence to the contrary. On the other hand, it is conceded that only concurrent verbalization can accurately reflect the mental status of participants (Bernardini 2001), and that post-hoc verbalization after long tasks may lead to incomplete descriptions (Ericsson and Simon 1984) and distortions. These limitations prompted Shlesinger to conclude that "for all intents and purposes, TAPs, in the ordinary sense, are not a viable tool for us" $(2000,3)$. In a more comprehensive methodological discussion of verbal methods in interpreting research, Ivanova (2000) suggests using transcripts of interpreters' output as retrieval cues during post-hoc verbalizations. This approach encourages participants to recall rather than to reconstruct cognitive processes, but does not solve the problem of a close interaction between participant and experimenter, which is argued to contaminate data (Bernardini 2001). Lamberger-Felber, who compares 12 interpreters' subjective assessment of source-text difficulty using Pöchhacker's (1994) discourse parameters, reports "high scoring variability in almost all individual parameters investigated" (Lamberger-Felber 2001,47). While these findings most likely indicate the need for further validation of Pöchhacker's taxonomy for the purpose of assessing the difficulty of source materials in simultaneous interpreting, they might also simply serve as a reminder to use caution when subjectively estimating the amount of load likely to be generated by a particular task.

4. As pointed out above, analytical approaches are unable to take into account individual differences: for the sake of the exercise it is thus assumed that the interpreter is unfamiliar with the name of the organizations he is working for or the venues he is working at and has to process the input incrementally without being able to resort to anticipation. 
Finally, subjectivity also makes its way into scientific inquiry through the backdoor of material selection for experimental purposes. Scholars select and match source materials for experiments on simultaneous interpreting and related tasks (i.e., shadowing, sight translation, simultaneous interpreting with text, etc.) based on a subjective assessment of difficulty. Readers are told, e.g., that the selected materials are "of roughly equal difficulty" (Moser-Mercer et al. 2000, 115) and thus left to trust the (interpreter-)researchers in their subjective assessment.

It would appear, then, that subjective methods might not provide the necessary objectivity to reliably assess cognitive load in simultaneous interpreting.

\subsection{Cognitive load in interpreting research: Performance methods}

Performance measures have a long tradition in psychology and made their way into interpreting research when the first psychologists started showing interest in this novel object of study. In line with the tradition at the time, the experimental analyses of the task focused on performance speed and performance accuracy. Oléron and Nanpon (1965), for example, quantified the time lag (i.e., EVS or Ear to Voice Span) between simultaneous interpreters and speakers, whereas Barik (1969) quantified errors and omissions in simultaneous interpreting.

It would appear that performance measures have lost little of their appeal as they are still frequently used measures in the experimental study of simultaneous interpreting (e.g., Mazza 2000; Gile 2008; 2011; Tauchmanová 2011). Although contributing to the generation of a non-negligible amount of data, it is interesting to see that one of the principal challenges inherent to performance methods (identified by Woodworth as early as 1899) has not often been addressed, let alone solved, in almost 50 years of experimental research on simultaneous interpreting: the trade-off between speed and accuracy. It is generally believed that, when carried out quickly, tasks will suffer in terms of accuracy, and when carried out accurately, they will suffer in terms of speed. The crux with simultaneous interpreting is that unlike in psychological experiments entailing arguably simpler tasks (like giving a true-or-false response), where participants can be instructed and given a regulatory focus, researchers do not usually attempt to control interpreters' regulatory focus. This means that the regulatory focus within one and the same interpreter might change from one performance to the next or even within one and the same performance. Furthermore, as simultaneous interpreting already consists of simultaneously executed tasks (i.e., language comprehension and language production), the traditional approach of having a main task carried out simultaneously with a secondary task (Paas et al. 2003; Haapalainen et al. 2010) is not practicable. In the absence of a traditional secondary task paradigm, establishing a causal relationship between performance speed or performance accuracy 
and cognitive load at any given point during the process becomes problematic. This problem is best exemplified by Pym's (2008) re-interpretation of Gile's (1999) data where, using a purely theoretical approach, Pym convincingly illustrates how varying priorities among participants can be invoked as an equally plausible explanation of the observed phenomena. ${ }^{5}$

Seeing that the aforementioned performance methods to measure cognitive load in simultaneous interpreting allow competing (and potentially mutually exclusive) interpretations of the results, it would seem prudent to combine them with other, more objective measures.

\subsection{Cognitive load in interpreting research: Psycho-psychological methods}

The fourth approach used to measure cognitive load relates to psycho-physiological techniques, i.e., methods allowing the measurement of cardiac, hematic, electro-dermal, ocular, muscular and cerebral responses. Many of these techniques are continuous and allow for a moment-to-moment analysis of events, which is of crucial importance for language processing, not only because cognitive load is assumed to fluctuate locally, but also because the individual operations inherent in language processing are performed very rapidly and can be measured in fractions of seconds (Mitchell 2004). Another advantage is that most physiological responses are controlled by the sympathetic nervous system, and as such, cannot be consciously influenced, but rather constitute an objective measure. The main drawback of psycho-physiological measures is the difficulty of identifying and determining what is actually measured. Furthermore, several techniques are invasive, and many of them highly complex and expensive. In order to be considered suitable for the measurement of cognitive load in simultaneous interpreting, any method must be evaluated against the following four requirements: noiseresistance $^{6}$, non-invasiveness ${ }^{7}$, temporal resolution and affordability. Given these constraints, it is not surprising that to date only very few psycho-physiological measures have been applied to the study of simultaneous interpreting. Petsche et al. (1993), for example, used electroencephalography (EEG) to compare brain activation as modulated by directionality (from or into the native language) during shadowing and simultaneous interpreting tasks. In order to avoid artifacts, however, both tasks had to be performed covertly (i.e., without articulation). Price et al. (1999) and Rinne et al. (2000) both used positron emission tomography (PET)

5. For a comprehensive discussion see Pym (2008).

6. The extent to which measurements are influenced and falsified by artifacts.

7. The extent to which a method invades the physical integrity of the body. 
to compare brain activation during translation and interpreting tasks. While Price et al. (1999) did not find any evidence for an increase in activation, Rinne et al. (2000) did. In both experiments, the method required the intravenous administration of ${ }^{15} \mathrm{O}-\mathrm{H}_{2} 0$, a positron-emitting tracer, making it rather invasive. More recently, Hervais-Adelman et al. (2011) used functional magnetic resonance imaging (fMRI) and successfully identified the neural substrates underlying the simultaneous processing of two languages during simultaneous interpreting. Among the chief limitations of this method is its temporal resolution (in the range of 2 to 3 seconds), which makes it impossible to time lock certain cognitive load phenomena. Pupillometry, finally, a method developed several decades prior to the advent of modern brain-imaging techniques, would appear to have better temporal resolution, be less invasive and more affordable than the aforementioned methods. To substantiate these claims the rest of the discussion will focus on this method and how it applies to the measurement of cognitive load in simultaneous interpreting.

\section{Pupillometry}

\subsection{The pupil}

If we exclude the effect of drugs, which can cause constriction (as is the case with alcohol, opioids, and antipsychotics) or dilation (in the case of the central nervous system stimulants and hallucinogens), the pupil, broadly speaking, reacts to three kinds of stimuli: luminosity (Clarke et al. 2003), emotions (Stelmack and Mandelzys 1975) and cognitive activity (Kahneman et al. 1969). These three stimuli can cause the pupil size to vary from $1.5 \mathrm{~mm}$ in bright light to up to 8 to $9 \mathrm{~mm}$ in dim light (Andreassi 2000). In order to make sense of the pupillary reactions and, more specifically, of the peak amplitude of the pupil, it is important to consider some of its fundamental physiological characteristics. First of all, pupil response can occur as quickly as $200 \mathrm{~ms}$ after stimulus presentation (Lowenstein and Loewenfeld 1962), although dilation as a response to cognitive load usually seems to begin after 300 to $500 \mathrm{~ms}$ (Beatty 1982; Hoeks and Levelt 1993). Lowenstein and Loewenfeld (1962) furthermore observed that pupil diameter is largest in rested individuals, whereas it decreases with fatigue. Similarly, it has been suggested that pupillary response decreases with age, weakening the correlation between cognitive load and pupil dilation (van Gerven et al. 2003). Another phenomenon to be kept in mind when interpreting pupillometric data is the manifestation of cognitive overload, i.e., when the task exceeds the cognitive resources available to perform it. Although Peavler (1974) suggests that once the capacity threshold has 
been reached, pupil dilation will stabilize, Poock (1973) and Granholm et al. (1996) found that pupil dilation decreases rapidly once cognitive overload was reached.

\subsection{The technology}

Since the initial interest in pupillometry in the 1960s, the technology used to measure pupil dilation has made considerable progress both in terms of accuracy and user-friendliness. In the early days of eye tracking ${ }^{8}$, subjects had to be "firmly positioned in an adjustable head-holder" (Bradshaw 1968,266), or use a "chin rest and bite board" (Schluroff 1982,137) to maintain the distance between the eye and the tracker. Pupil size was then recorded with an external video camera, and the film was either plotted onto graph paper or projected onto a larger surface frame by frame; the pupil diameter was measured manually (see Bradshaw 1968). Today, pupillometry is considered a relatively simple, affordable and non-invasive method for assessing autonomic function (Bär et al. 2005), and is applied in psychophysiology, pharmacology, neurology, and psychiatry. While until recently, only fixed (or head-mounted) eye trackers were deemed suitable for the measurement of pupil dilation, Klingner et al. (2008) convincingly replicated some of the classic cognitive pupillometry results (e.g., Kahneman and Beatty 1966) using a remote eye tracker. ${ }^{9}$

\subsection{Using pupillometry to measure cognitive load in simultaneous interpreting}

From the preceding discussion one might conclude that pupillometry is ideally suited to measure cognitive load during a complex task like simultaneous interpreting. It might surprise, then, to see that it has only been applied to the interpreting paradigm very rarely. In a groundbreaking experiment, Tommola and Niemi (1986) used pupillometry to study the effect of directionality-contingent syntactic complexity on mental load, for the first time demonstrating the feasibility of the method. Almost a decade later, Hyönä et al. (1995) conducted a more systematic analysis of cognitive load comparing and contrasting the load generated during different (arguably related) language processing tasks (i.e., listening comprehension, shadowing, and simultaneous interpreting). The results indicated an increase of mean pupil dilation, and consequently cognitive load, from listening comprehension to shadowing, and simultaneous interpreting. Seeber and Kerzel used the same method to measure online (i.e., real time) cognitive load during

8. Pupil dilation is usually measured using an eye tracker, i.e. an infrared camera measuring the movement of the eyes.

9. See Klingner (2010) for a detailed description of the methodology including data processing. 
simultaneous interpreting and gathered evidence suggesting that German verbfinal structures generate more cognitive load than German verb-initial structures when interpreted into English (Seeber and Kerzel 2012). Their data also suggests that simultaneously interpreting sentences without context generates more cognitive load than interpreting sentences (ceteris paribus) embedded in context. These examples illustrate the great potential of pupillometry as a method and t TEPRs as a measure of cognitive load in simultaneous interpreting.

\subsection{Potential and limitations of pupillometry in simultaneous interpreting}

Like all research methods, pupillometry has its potential and limitations. Its potential, largely due to its limited invasiveness and its high temporal resolution, has already been discussed in the previous sections. As for its limitations, they mainly stem from the intrinsic nature of the measures it provides. Mean dilation, peak dilation and mean latency are revealing when applied to short auditory stimuli (e.g., at the phrase and sentence level), but much less so when applied to long stimuli (e.g., at the discourse level). In fact, Schultheis and Jameson (2004) found no difference in mean pupil dilation across (340-word long) texts of different (subjective) difficulty. Their conclusion that "pupil size may differ between easy and difficult conditions only in certain periods of a task" $(2004,233)$ is supported by Haapalainen et al. (2010), who found that median heart flux and median electrocardiogram are more reliable measures of task difficulty than median pupil dilation with a trial length of three minutes. These findings, it seems, are related to the very nature of the measure that reflects moment-to-moment variations of load and maps several sources of load onto one measure. Averaging changes in pupil dilation over long periods (e.g., of several minutes) might thus cancel out the changes in cognitive load reflected in them. It is no coincidence that the experiments in which this method has been applied successfully (both outside and within the paradigm) were tightly controlled and used isolated stimuli and short periods of interest (e.g., Hyönä et al. 1995; Seeber and Kerzel 2011). This suggests that, provided the necessary methodological rigor is applied, pupillometry might be a reliable method to measure local cognitive load in simultaneous interpreting.

\section{Conclusion}

In this chapter I attempted to briefly illustrate, by means of a few examples, how different methods have been applied to researching cognitive load in simultaneous interpreting. Although each of the four approaches has its unique advantages and disadvantages, this analysis suggests the use of pupillometry as a way to observe 
more objectively how much cognitive load is generated during the interpreting task as it unfolds. Having said that, even this measure currently comes with as of yet unresolved challenges. In fact, it would appear that while reliable as a means to assess local cognitive load at or below the sentence level, this method might not be indicated to quantify average cognitive load across long stimuli. What is more, the signal-to-noise ratio ${ }^{10}$ requires comprehensive data preparation (see Klingner et al. 2008) while the many-to-one mapping still precludes us from attributing measured load to individual component tasks. Much as with other limitations identified in this overview, they are not to suggest that the method is unsuitable or invalid. However, it would appear that we do require more "research into research" and that, "the day when we can spend more time discussing what we found, and less time agonizing over how we found it or whether we went about it the right way" (Shlesinger 2000, 13) has not yet come.

\section{References}

Andreassi, John L. 2000. Psychophysiology: Human Behavior and Physiological Response, 4th ed. Mahwah, NJ: Lawrence Erlbaum.

Bär, Klaus-Jürgen, Michael K. Boettger, Silke Till, Julia Dolicek, and Heinrich Sauer. 2005. "Lateralization of Pupillary Light Reflex Parameters." Clinical Neuropsychology 116: 790798.

Barik, Henri C. 1969. A Study of Simultaneous Interpretation. Unpublished Ph.D. dissertation, University of North Carolina, Chapel Hill.

Beatty, Jackson. 1982. “Task Evoked Pupillary Responses, Processing Load, and the Structure of Processing Resources.” Psychological Bulletin 91 (2): 276-292.

DOI: 10.1037/0033-2909.91.2.276

Bernardini, Silvia. 2001. "Think-Aloud Protocols in Translation Research: Achievements, Limits, Future Prospects.” Target 13 (2): 214-263. DOI: 10.1075/target.13.2.03ber

Bradshaw, John L. 1968. "Load and Pupillary Changes in Continuous Processing Tasks." British Journal of Psychology 59 (3): 265-271. DOI: 10.1111/j.2044-8295.1968.tb01139.x

Clarke, Robert J., Hongyu Zhang, and Paul D.R. Gamlin. 2003. "Characteristics of the Pupillary Light Reflex in the Alert Rhesus Monkey." Journal of Neurophysiology 89: 3179-3189.

DOI: $10.1152 /$ jn.01131.2002

de Groot, Annette M.B. 1997. “The Cognitive Study of Translation and Interpretation. Three Approaches." In Cognitive Processes in Translation and Interpreting, ed. by Joseph H. Danks, Gregory M. Shreve, Stephen B. Fountain, and Michael K. McBeath, 25-56. Thousand Oaks, CA: Sage.

de Groot, Annette M.B. 2000. “A Complex-Skill Approach to Translation and Interpreting.” In Tapping and Mapping the Processes of Translation and Interpreting, ed. by Sonja Tirkkonen-

10. The level of desired signal compared to the level of background noise (or artifacts). 
Condit, and Riitta Jääskeläinen, 53-68. Amsterdam: John Benjamins.

DOI: 10.1075/btl.37.06gro

Ericsson, Anders K., and Herbert A. Simon. 1984. Protocol Analysis: Verbal Reports as Data. Cambridge, MA: Bradford Books/MIT Press.

Gile, Daniel. 1995. Regards sur la recherche en interprétation de conférence. Lille: Presses universitaires de Lille.

Gile, Daniel. 1997. “Conference Interpreting as a Cognitive Management Problem.” In Cognitive Processes in Translation and Interpreting, ed. by Joseph H. Danks, Stephen B. Fountain, Michael K. McBeath, and Gregory M. Shreve, 196-214. Thousand Oaks, CA: Sage.

Gile, Daniel. 1999. “Testing the Effort Models' Tightrope Hypothesis in Simultaneous Interpreting - A Contribution." Hermes 23: 153-172.

Gile, Daniel. 2008. "Local Cognitive Load in Simultaneous Interpreting and its Implications for Empirical Research.” Forum 6 (2): 59-77.

Gile, Daniel. 2011. "Errors, Omissions and Infelicities in Broadcast Interpreting: Preliminary Findings from a Case Study." In Methods and Strategies of Process Research, ed. by Birgitta Englund Dimitrova, Cecilia Alvstad, Adelina Hild, and Elisabet Tiselius, 201-218. Amsterdam: John Benjamins. DOI: 10.1075/btl.94.15gil

Gopher, Daniel, and Rolf Braune. 1984. "On the Psychophysics of Workload: Why Bother with Subjective Measures?” Human Factors 26: 519-532.

Granholm, Eric, Robert F. Asarnow, Andrew J. Sarkin, and Karen L. Dykes. 1996. "Pupillary Responses Index Cognitive Resource Limitations.” Psychophysiology 33: 457-461.

DOI: 10.1111/j.1469-8986.1996.tb01071.x

Haapalainen, Eija, SeungJun Kim, Jodi F. Forlizzi, and Anind K. Dey. 2010. "Psycho-Physiological Measures for Assessing Cognitive Load.” In Proceedings of the 12th International Conference on Ubiquitous Computing, 301-310. New York: ACM.

Hervais-Adelman, Alexis G., Barbara Moser-Mercer, and Narly Golestani. 2011. "Executive Control of Language in the Bilingual Brain: Integrating the Evidence from Neuroimaging to Neuropsychology." Frontiers in Psychology 2 (234): 1-8. DOI: 10.3389/fpsyg.2011.00234

Hoeks, Bert, and Willem J.M. Levelt. 1993. "Pupillary Dilation as a Measure of Attention: A Quantitative System Analysis." Behavior Research Methods, Instruments and Computers 25: 16-26. DOI: 10.3758/BF03204445

Hyönä, Jukka, Jorma Tommola, and Anna-Mari Alaja. 1995. "Pupil Dilation as a Measure of Processing Load in Simultaneous Interpreting and Other Language Tasks." The Quarterly Journal of Experimental Psychology 48A (3): 598-612. DOI: 10.1080/14640749508401407

Ivanova, Adelina. 2000. “The Use of Retrospection in Research on Simultaneous Interpreting." In

Tapping and Mapping the Processes of Translation and Interpreting, ed. by Sonja TirkkonenCondit, and Riitta Jääskeläinen, 27-52. Amsterdam: John Benjamins.

DOI: 10.1075/btl.37.05iva

Kahneman, Daniel. 1973. Attention and Effort. Englewood Cliffs, NJ: Prentice-Hall.

Kahneman, Daniel, and Jackson Beatty. 1966. "Pupil Diameter and Load on Memory." Science 154: 1583-1585. DOI: 10.1126/science.154.3756.1583

Kahneman, Daniel, Bernard Tursky, David Shapiro, and Andrew Crider. 1969. "Pupillary, Heart Rate, and Skin Resistance Changes during a Mental Task.” Journal of Experimental Psychology 79 (1): 164-167. DOI: 10.1037/h0026952

Klingner, Jeff. 2010. Measuring Cognitive Load during Visual Tasks by Combining Pupillometry and Eye Tracking. Unpublished Ph.D. dissertation, Stanford University Computer Science Department. 
Klingner, Jeff, Rakshit Kumar, and Pat Hanrahan. 2008. "Measuring the Task-Evoked Pupillary Response with a Remote Eye Tracker." ETRA 2008: Proceedings of the 2008 Symposium on Eye Tracking Research and Applications, 69-72. Savannah, GA: ACM.

Lamberger-Felber, Heike. 2001. “Text-Oriented Research into Interpreting: Examples from a Case-Study." Hermes 26: 39-64.

Lowenstein, Otto, and Irene E. Loewenfeld. 1962. “The Pupil." In The Eye, Vol. 3, Muscular Mechanisms, ed. by Hugh Davson, 231-267. New York: Academic Press.

Massaro, Dominic W., and Miriam Shlesinger. 1997. "Information Processing and a Computational Approach to the Study of Simultaneous Interpretation." Interpreting 1 (1/2): 13-53. DOI: 10.1075/intp.2.1-2.02mas

Mazza, Cristina. 2000. Numbers in Simultaneous Interpretation. Unpublished graduation thesis, Universita degli Studi di Bologna, SSLMIT, Forli.

Miller, George A. 1956. “The Magical Number Seven, Plus or Minus Two: Some Limits on our Capacity for Processing Information.” Psychological Review 63: 81-97.

DOI: $10.1037 / \mathrm{h} 0043158$

Mital, Anil, and Majorkumar Govindaraju. 1999. "Is It Possible to Have a Single Measure for all Work?” International Journal of Industrial Engineering Theory 6: 190-195.

Mitchell, Don. 2004. "On-line Methods in Language Processing: Introduction and Historical Review." In The On-line Study of Sentence Comprehension: Eyetracking, EPRs and Beyond, ed. by Manuel Carreiras, and Charles Clifton Jr., 15-32. Hove: Taylor \& Francis.

Moser-Mercer, Barbara. 1997. "Beyond Curiosity. Can Interpreting Research Meet the Challenge?” In Cognitive Processes in Translation and Interpreting, ed. by Joseph H. Danks, Stephen B. Fountain, Michael K. McBeath, and Gregory M. Shreve, 176-195. Thousand Oaks, CA: Sage.

Moser-Mercer, Barbara, Ulrich Frauenfelder, Beatriz Casado, and Alexander Künzli. 2000. "Searching to Define Expertise in Interpreting." In Language Processing and Simultaneous Interpreting, ed. by Birgitta Englund Dimitrova, and Kenneth Hyltenstam, 107-132. Amsterdam: John Benjamins. DOI: 10.1075/btl.40.09mos

Oléron, Pierre, and Hubert Nanpon. 1965. "Recherches sur la traduction simultanée." Journal de psychologie normale et pathologique 62: 73-94.

Paas, Fred G.W.C., and Jeroen J.G. Merrienboer. 1993. “The Efficiency of Instructional Conditions: An Approach to Combine Mental Effort and Performance Measures." Human Factors 35: 737-743.

Paas, Fred G.W.C., Juhani E. Tuovinen, Huib K. Tabbers, and Pascal W.M. van Gerven. 2003. "Cognitive Load Measurement as a Means to Advance Cognitive Load Theory." Educational Psychologist 38 (1): 63-71. DOI: 10.1207/S15326985EP3801_8

Peavler, Scott W. 1974. "Pupil Size, Information Overload, and Performance Differences." Psychophysiology 11: 559-566. DOI: 10.1111/j.1469-8986.1974.tb01114.x

Petsche, Hellmuth, Susan C. Etlinger, and Oliver Filz. 1993. "Brain Electrical Mechanisms of Bilingual Speech Management: An Initial Investigation.” Electroencephalography and Clinical Neurophysiology 86: 385-394. DOI: 10.1016/0013-4694(93)90134-H

Pöchhacker, Franz. 1994. Simultandolmetschen als komplexes Handeln. Tübingen: Gunter Narr. Poock, Gary K. 1973. “Information Processing vs. Pupil Diameter." Perceptual and Motor Skills 37: 1000-1002. DOI: 10.2466/pms.1973.37.3.1000

Price, Cathy J., David W. Green, and Roswitha Von Studnitz. 1999. "A Functional Imaging Study of Translation and Language Switching." Brain 122 (12): 2221-2235.

DOI: $10.1093 /$ brain/122.12.2221 
Pym, Anthony D. 2008. "On Omission in Simultaneous Interpreting: Risk Analysis of a Hidden Effort." In Efforts and Models in Interpreting and Translation Research: A Tribute to Daniel Gile, ed. by Gyde Hansen, Andrew Chesterman, and Heidrun Gerzymisch-Arbogast, 83105. Amsterdam: John Benjamins DOI: 10.7202/1011268ar

Rinne, Juha O., Jorma Tommola, Matti Laine, Bernd J. Krause, Daniela Schmidt, Valtteri Kaasinen, Mika Teräs, Hannu Sipilä, and Marianna Sunnari. 2000. “The Translating Brain: Cerebral Activation Patterns During Simultaneous Interpreting.” Neuroscience Letters 294: 85-88. DOI: 10.1016/S0304-3940(00)01540-8

Shlesinger, Miriam. 2000. "Interpreting as a Cognitive Process: How can we Know what Happens?" In Tapping and Mapping the Processes of Translation and Interpreting, ed. by Sonja Tirkkonen-Condit, and Riitta Jääskeläinen, 3-15. Amsterdam: John Benjamins.

DOI: 10.1075/btl.37.03shl

Schluroff, Michael. 1982. "Pupil Responses to Grammatical Complexity of Sentences." Brain and Language 17: 133-145. DOI: 10.1016/0093-934X(82)90010-4

Schultheis, Holger, and Anthony Jameson. 2004. "Assessing Cognitive Load in Adaptive Hypermedia Systems: Physiological and Behavioral Methods." In Adaptive Hypermedia and Adaptive Web-Based Systems: Proceedings of AH 2004, ed. by Wolfgang Nejdl, and Paul De Bra, 225-234. Berlin: Springer. DOI: 10.1007/978-3-540-27780-4_26

Seeber, Kilian G. 2007. "Thinking Outside the Cube: Modeling Language Processing Tasks in a Multiple Resource Paradigm.” Interspeech, Antwerp, Belgium. 1382-1385.

Seeber, Kilian G. 2011. "Cognitive Load in Simultaneous Interpreting: Existing Theories - New Models.” Interpreting 13 (2): 176-204. DOI: 10.1075/intp.13.2.02see

Seeber, Kilian G., and Dirk Kerzel. 2012. "Cognitive Load in Simultaneous Interpreting: Model Meets Data." International Journal of Bilingualism 16 (2): 228-242.

DOI: $10.1177 / 1367006911402982$

Setton, Robin. 2001. “Translation Studies and Cognitive Science: Do We Need Each Other?” CTIS Occasional Papers 1: 113-126.

Setton, Robin. 2003. "Models of the Interpreting Process." In Avances en la investigación sobre la interpretación, ed. by Angela Collados Aís, and José Antonio Sabio Panilla, 29-91. Granada: Editorial Comares.

Stelmack, Robert M., and Nathan Mandelzys. 1975. "Extraversion and Pupillary Response to Affective and Taboo Words." Psychophysiology 12: 536-540.

DOI: 10.1111/j.1469-8986.1975.tb00042.x

Tauchmanová, Jana. 2011. Daniel Gile's Effort Model in Simultaneous Interpreting. Unpublished MA thesis, Institute of Translation Studies, Charles University, Prague.

Tommola, Jorma, and Pekka Niemi. 1986. "Mental Load in Simultaneous Interpreting: An Online Pilot Study." In Nordic Research in Text Linguistics and Discourse Analysis, ed. by Lars S. Evensen, 171-184. Trondheim: Tapir.

Van Gerven, Pascal W.M., Fred Paas, Jeroen J.G. van Merrienboer, and Henk Schmidt. 2003. "Memory Load and the Cognitive Pupillary Response in Aging." Psychophysiology 41: 167174. DOI: 10.1111/j.1469-8986.2003.00148.x

Wickens, Christopher D. 1984. “Processing Resources in Attention.” In Varieties of Attention, ed. by Raja Parasuraman, and David R. Davies, 63-102. New York: Academic Press.

Wickens, Christopher D. 2002. "Multiple Resources and Performance Prediction." Theoretical Issues in Ergonomics Science 3 (2): 159-177. DOI: 10.1080/14639220210123806

Woodworth, Robert S. 1899. “The Accuracy of Voluntary Movement.” Psychological Review 3 (Suppl. 13): 1-119. 
Yin, Bo, Fang Chen, Natalie Ruiz, and Eliathamby Ambikairajah. 2008. "Speech-Based Cognitive Load Monitoring System.” Proceedings of the IEEE International Conference on Acoustics, Speech and Signal Processing, 2041-2044. Las Vegas, Nevada. 


\title{
Extended translation
}

\section{A sociocognitive research agenda}

\author{
Hanna Risku and Florian Windhager \\ University of Graz, Austria / Danube University Krems, Austria
}

\begin{abstract}
Consideration of current developments in cognitive science is indispensable when defining research agendas addressing cognitive aspects of translation. One such development is the recognition of the extended nature of human cognition: Cognition is not just an information manipulation process in the brain, it is contextualised action embedded in a body and increasingly mediated by technologies and situated in its socio-cultural environment. Parallel developments are found in neighbouring disciplines, such as sociology with its actor-network and activity theories. This chapter examines these approaches, their shared methodological tenets (i.e., ethnographic field studies) and the implications of the situated cognition approach for describing the cognitive aspects of translation, using a translation management case study to discuss conceptual and methodological issues.
\end{abstract}

Keywords: extended translation, situated cognition, extended cognition, sociocognition, actor-network theory, activity theory, translation culture, translation management, specialization, networking

\section{Introduction}

This chapter discusses concepts used in the relatively young paradigms of situated and distributed cognition and examines their implications for research into the cognitive aspects of translation. Cognitive science has seen the development of notable extensions within the last decades by emphasising the increasing importance of the social and physical environment. Viewed from this perspective, cognition is the result of the constant interaction between people and their social and material environments - a distributed and highly adaptive process, which weaves - and is woven by - networks of actors and artefacts.

To unlock and develop these insights for translation studies, we seek to complement the situative approach in cognitive science with two strongly resonant theoretical approaches in sociology and psychology, namely actor-network theory 
(ANT; Latour 1986; 2005) and activity theory (AT; Leontiev 1978). There are a number of parallel developments in these approaches, which have the potential to make a valuable contribution to translation studies, particularly when it comes to the study of the extended cognitive aspects of translation.

In doing so, we use a case study to illustrate what we refer to as "translation in the wild", i.e., situated translation in practice (see also Martín de León 2008). This example is an ethnographic study of the evolving network of actors, artefacts, roles and patterns of work coordination among translation project managers in a translation services company. We examine which conceptual instruments situated cognition, ANT and AT provide us with to describe the case studied and interpret the ethnography of the changes observed.

As Vorderobermeier (2008, 38; with reference to Simeoni 1998, 3) notes, the views taken in cognitive translation studies and translation process research often appear strangely "culture independent" and "ahistorical": the cognitive basis and processes of translation are described as if they were universal, context-independent procedures (e.g., referring to the general differences between translation students and experienced translators, regardless of culture, time and place). This is of course inevitable when it comes to limiting the number of variables in an analysis on the micro-level of cognition and thus conforming with the scientific ideal of hypothesis testing in controlled experiments. However, the current strong focus on micro-level analyses is remarkable in a discipline like translation studies, where so much emphasis is otherwise placed on the dependence of human understanding and action on culture, situation and context. In cognitive translation studies, as in any other explanatory approach to socially embedded behavior, distinctions are made between micro- and macro-level analysis, laboratory and field-based methods as well as between bottom-up and top-down explanations. We view these apparent dichotomies as complementary perspectives and aim in this chapter to help explain the nested and embedded nature of translatorial cognition and action (Risku et al. 2013).

To do justice to the fact that cognitive translation processes also develop within specific cultures and are dependent on the place and time of this development, we would like to draw attention to and emphasise the relevance of the term 'translation culture' introduced by Prunč $(2007,24-25)$. Prunč defines translation culture as the self-regulating translation-related subsystem in a culture that has developed historically out of initially unstructured fields of translation practice. A translation culture can be differentiated into different subcultures (Prunč 2007, 25). The study of culture is a sociological and cultural-science undertaking, but it is also a cognitivescience task, since the key factors in human intelligence and the human capacity act as well as collective and communicative action and the signs, tools and technologies involved are also clearly objects of extended cognitive science research. 
Unfortunately, we cannot deal with the definition of the concept of 'culture' in any detail here. But we would like to note that the situated, embodied cognition view taken in this chapter is not compatible with the classic definitions of culture which list the norms, conventions, values, expectations and patterns supposedly shared by a community or a group of people. Instead, we need a more interactive and dynamic concept (e.g., Sperber and Hirschfeld 1999, Oyserman 2011) that includes aspects like how artefacts (e.g., texts) are cultivated, the tools and technologies used in the process and the way things are done and achieved collectively.

\section{Extended perspectives on cognition and action}

A key focus in contemporary cognitive science is on the study of the situated and distributed nature of human behaviour and thought. In other words, context is now increasingly seen and accepted as an important part and aspect of the cognitive process. Cognition is not just an information processing activity in isolated brains; it is a context-dependent interaction of mind, body and environment. This is why the distributed and situative cognition approaches (embodied/embedded cognition/action, extended cognition) pay particular attention to the dynamic and situative aspects of cognition as interaction.

\subsection{Situated cognition}

The new cognitive science movements emphasise the relevance of the social and physical environment (Clark 1997; 2008; Clark and Chalmers 1998). On a network level, cognition incorporates the dynamic interplay of artefacts, workplaces and spatial context, as well as socio-cultural spaces in different environments, which are increasingly mediated by information and media infrastructures.

The influence of these environments is so extensive that the behaviour and thoughts of singularly focused actors appear unforeseeable: individual expectations, values and accustomed patterns of behaviour are only initial hypotheses which can be shaped by the actual reality of the here and now. We are therefore influenced far more by our present situation than was previously assumed (Menary 2010). While this relativises the conclusions that can be drawn from certain kinds of observations (e.g., introspective explanations of behaviour in think-aloud protocols), it also strengthens the relevance of the targeted design of contexts and environments. The relevance of culture as a defining principle of the socio-material environment and of personal behaviour is thus also accorded increasing importance in cognitive science.

By necessity, these changes on a conceptual level are followed by adaptations of approaches. Traditional foci on output and content parameters of isolated 
phenomena in laboratory settings are extended to the observation of dynamic sequences of actions in which people interact with their environment and the artefacts it contains. Social processes and embeddedness now also take a central role in observation and analysis.

The methods used include ethnographic, participative observations of real situations, cooperations and processes. To emphasise the difference between these methods and the laboratory tests and examinations of cognitive products, Hutchins (1995a), a pioneer of the distributed cognition approach, named the object of his research "cognition in the wild". Hutchins experimented with the thought of viewing the cockpit of an aircraft with its instruments and (co-)pilots as an interrelated cognitive unit. He described "how a cockpit remembers its speeds" (1995b) and showed that cognition can be understood as the interplay of multiple dynamic systems, i.e., pilots, instruments, aircraft, ground control and the surrounding airspace. In this extended view of cognition, former boundary conditions or environmental factors serve not only as contingent (and thus nonessential?) external circumstances in studies of the cognitive aspects of translation processes, they are also integral parts of the cognition and translation processes themselves. Hence, as a methodological consequence, extended cognition studies inevitably follow "leaking minds" into their social and technical environments, thereby including process, interaction and artefact analysis into a combined and linked view on dynamic complexity.

\subsection{Actor-network theory}

Exciting parallels can be found in science and technology research, where actornetwork theory (ANT) is following a similar route (Latour 2005). According to Latour, social processes (like the dynamics of innovation and stabilisation) can only be adequately understood through the meticulous observation of interactions between human actors and non-human artefacts - referred to collectively as "actants". The goal of this hybrid approach is to develop a theoretical language that allows a better description of the omnipresent socio-technical actant assemblies by taking a balanced account of the interdependencies and interactions between people and things. In translation practice, this would mean looking not only at the translators, but also at the other parties involved in the translation process (clients, subject-matter experts, colleagues) and the many tools that are part of modern translation work (text processors, online research tools, translation memories).

By carefully tracking and following the dynamics of actant constellations, academic domains like psychology, sociology, technology and media studies must frequently be crossed. In return, a more integrated view of complex, interrelated phenomena in socio-technical behaviour can be expected. Latour refers to the 
medium and long-term innovation and transformation processes that become visible in such studies using a generalised concept of "translation" (of course, the "translation" he refers to is something quite different from the concept common in translation studies). ANT as a "sociology of translation" hence follows the emergence and stabilisation of facts and artefacts by observing and tracking different stages of problematisation (problems are addressed), "interessement" (other actors gaining interest), enrolment (actors are enrolled into an innovation or production process), and mobilisation of allies (further support for stabilisation by mobilising others; Callon 1986). From the early stage of such a process until its growth and stabilisation, references and inscriptions - text, images, technical and other artefacts produced in the work process - are circulated through the network, transforming actants and becoming transformed in themselves (Latour 1986; see also Buzelin 2005, 198).

From this perspective, translatory text production re-appears as an actor network by itself and as a main agency in multicultural fields where the transcultural extension and mediation of actor networks must be accomplished. This approach has been adapted and elaborated for the translation field, for example, by Buzelin (2005), Bogic (2010) and Abdallah (2011).

Latour (2005) and Hutchins (1995a, b) base their research on ethnographic methods and/or ethnomethodology: on participative or detailed historical observation of authentic production and application situations - at the actual spot where the actors are situated - and on the analysis of the material (texts, instruments) and immaterial objects (e.g., memorised checklists) which are formed by and contribute to forming the network. They partly share their underlying theoretical approach with the 'third member of the gang', namely activity theory.

\subsection{Activity theory}

Activity theory (AT) is an interdisciplinary approach with an applied focus on the study and development of systems of computer-supported cooperative work (CSCW; Engeström 1990; Kuutti 1991a,b). Similar to situated cognition, AT emerged as a theoretical framework from psychological research by recognising the importance of the situated, object-oriented and tool-mediated aspect of human activities (Leontiev 1978). Human action thus is seen as goal-directed, purposeful interaction of a subject with an object (which could be another subject or subjects) through the use of a tool or artefact (including signs and languages) - a setting that varies greatly in its cultural and historical situatedness. Collective and individual actors find themselves situated in - and faced with - object environments which embrace natural entities and socio-culturally shaped or produced artefacts, including signs and symbol systems (Kaptelinin and Nardi 2006). The source text, 
research material, parallel texts and many versions of the target text that are emergent in the translation process would be a case in point here.

Activities can be reconstructed as procedures and ways to act on, cultivate, use, transform, connect or maintain these objects, or to solve problems arising from these constant (inter)actions. To do so, developed cultures rely on the use of tools and technologies. Generally speaking, tools can be described as internal or external means of transforming material or immaterial objects of various kinds. Material tools like hammers, scalpels, machines and computers and immaterial tools like concepts, logics and scientific methods are used to transform external objects and inner dispositions. According to the basic principle of AT, "continuous development", tools develop over time and incorporate the former interactions of actors with their environments. By collectively operating in their environments of objects and tools, actors also transform themselves - along with their guiding programmes of action. Agricultural or house building activities and intercultural communication activities and translatory action offer good illustrations of this point. For example, there is a certain translation concept that is inherent in translation memories, namely that any source-text segment can potentially be replaced with the corresponding target-text segment used in a previous translation. Introducing translation memories implicitly suggests introducing this concept in practice, thus guiding the practice of translation in a certain direction. The tool was developed according to a particular view of the cognitive process of translation, and no matter what the real cognitive processes, it has the potential to discreetly but firmly guide the cognitive processes in that direction.

Whether translation is seen as professional text production (Schäffner 1997), text design (Holz-Mänttäri 1993) or the mediation of transcultural communication (Snell-Hornby, Jettmarová and Kaindl 1997), AT helps to identify the tools and means used to pursue the intended goals of translation activities, to understand how they are collectively operated and to see how these tools and activity patterns develop over time.

Since AT emphasises human intention and the asymmetry of people and things, it is clearly at odds with ANT and also partly with situated and distributed cognition (Kaptelinin and Nardi 2006, 10). However, with its notion of culture and technology shaping human activity, it is in fact in line with the other approaches mentioned above, and (more importantly in our case) its methodological approach shows a similar reorientation towards (1) extending the scope of analysis from individuals and systems/texts to subjects in the social and material tool-mediated world, (2) observing real-life situations instead of using formal models or lab studies, and doing so ideally in (3) long-term studies that can show the development and changes in the subjects, technologies, interaction and overall context (Kaptelinin and Nardi 2006, 35). 
To outline a setting in which this framework can be leveraged to guide research into translatory action in the wild, we will now introduce an empirical extended cognition and CSCW case study carried out in the translation studies field (for a comprehensive description of the study, see Risku 2010). This field study was carried out with the aim of observing and understanding the role of cooperation and technology in the cognitive activities of project managers in translation agencies. We did not explicitly follow the ANT or AT approaches in the study, but motivated the ethnographic study design by referring to CSCW and the situated cognition approach. Thus, the examination of the relevance and application of the conceptual instruments in these three approaches forms the retrospective 'postproject' view we want to take in the present chapter.

\section{Empirical case study: changes in translation management}

In 2001 and 2002, we carried out our first studies in the field of translation: an ethnographic field study and artefact analysis. We then conducted a follow-up study in 2007 to examine the changes that had taken place in the meantime. This follow-up study focused primarily on the changes in the way project managers in a translation company work. More specifically, we observed everyday working life in a translation company in Vienna for four weeks for the first study and for one week (five years later) for the second study. We also carried out an artefact analysis at both points in time. Our goal was to describe one particular case in all its uniqueness in order to understand possible developments and enrich our scientific explanatory models (cf. Susam-Sarajeva 2009, 48; Donmoyer 2000, 63). The translation company studied specialises in expert communication and technical communication. The production of the target texts - the actual translations - is outsourced to translation agencies and specialised translators around the world. The majority of its customers are large firms with a regular need for translations into several target languages and for several target markets.

\subsection{Main findings}

Our analysis reconstructed salient, observable changes in the period between the two studies, four of which we would like to describe in more detail:

1. Expansion in terms of headcount and office space. The headcount has grown from five to 12 people, and a new hierarchical role level has been introduced. Increased professionalisation and specialisation in work distribution can thus be observed. The office space has grown from two to four rooms. This has 
reduced direct personal communication and led to staff phoning or sending internal e-mails to colleagues in other rooms, thus increasing the level of indirect and mediated communication.

2. Concentration on core business. A change in strategy has been introduced, and the company now focuses even more strongly than before on its core business - translation project management. The production and review of translations is consistently outsourced to freelancers and translation agencies instead of being carried out in-house. This change again strengthens the need for specialisation as well as coordination with external collaboration partners like freelance translators and reviewers.

3. Introduction of a project management system. A customised translation specific project management software has been installed, bringing with it far-reaching changes in the way the translation company works.

a. The project management software defines the project steps. The workflow and almost all order documentation and versions of texts are handled electronically in the system. Electronic media — primarily e-mail — are now used far more frequently in communication with clients, translators, technology advisors and other partners.

b. The translation languages offered have remained the same, while the company's languages of business have been reduced to German and English to accommodate the software. This has decreased the linguistic and cultural variety in day-to-day communication. It has also 'de-personalised' communication, which had previously been kept as personal and individual as possible.

c. The artefact analysis that formed part of the 2002 study included a wide range of physical and electronic artefacts; five years later, analogue media had clearly taken a back-seat role. While five years ago some source texts were still delivered in paper format (by post or fax), they are now sent only in electronic format. While completed work at the end of the day had previously been signified by a lack of paper and files on the left side of a desk, the goal now is to leave the office with an empty e-mail inbox. Interesting exceptions here are reminders and to-do lists or overviews, which are generally still noted or jotted down on paper.

d. Translation specific information and communication technologies (ICTs) had already played a major role at the time of the first study and were seen then as an "asset". At that time, the managing director described the company's translation memory as a "real treasure". She now views it as an "absolute prerequisite" for their work and notes that her "biggest nightmare is the thought of a system crash, because then everything would come to a standstill, like it would for an airline". The use of such ICTs has 
also resulted in new selection criteria for suppliers: the penetration of media into translation has placed new requirements on external translators. Consequently, the company now only outsources to freelancers with adequate media skills. Websites and online communities serve as indications of such abilities: translators should, for example, have their own website and be active in online translation networks. As a result, the role of online networks has changed from that of voluntary, informal platforms to a business necessity.

The general changes or trends observed can essentially be grouped into three key areas:

1. Specialisation: increased professionalisation and specialisation in work distribution.

2. Networking: increased relevance of external cooperation partners, leading to extended integration efforts by means of computer supported collaborative work.

3. Digitalisation: increased dependence on translation specific ICTs (including a shift towards screen-based work) and an increased proportion of indirect, electronic communication.

\subsection{Frameworks and concepts for interpreting the findings}

How can the dynamics in this translation network be interpreted in relation to the frameworks outlined above? Which conceptual instruments do situated cognition, ANT and AT provide us with to describe the translation culture studied and to interpret the ethnography of the cultural changes? They clearly correspond in two aspects: (1) emphasis on social embeddedness, and (2) increasing recognition of artefacts and technologies as key elements in culture and cognition.

If we pursue a representation of translation that is consistent with ANT or extend the parallels of 'extended cognition' to 'extended translation', we can include key artefacts of translatory action as independent elements into the network. Consequently, the continuous (co-)development of the sociocognitive interaction patterns (on the human level in the actor network) could be re-interpreted with regard to evolving ICT networks and technologies. The fact that the company in our study was prepared to accept a protracted adaptation of the translation project management software to suit its own purposes suggests a trend towards greater technologisation. Similarly, the fact that the translation memory was already described by the managing director as a "treasure" at the time of the first study offers clear support for the systemic amalgamation of human cognition and memory operations with the disburdening, supporting and catalysing functions 
of information and translation technology. There is even a strong interdependence between the cognitive translation processes and the artefacts. The project management and translation support systems used can be described as a technical network, with both - technology and network - coming together here as one. Technology and network are a collective "assemblage" (Latour 2005) that would no longer even function as a non-combination, non-network, or non-collective - hence the thought of a system crash being the managing director's worst nightmare.

Building on this hybrid infrastructure, translation norms and translations are "collective constructions involving the participation of multiple mediators" (Buzelin 2005, 199, with reference to Latour 2005 and Bourdieu 2001, 93). The translation process is now increasingly mediated through different people and instruments, making the chain longer and longer and the network increasingly larger and more complex. Orders are initiated in one place, defined and allocated in another, then analysed, calculated and prepared in a third; they are also analysed, calculated and pre-translated using technology tools; translators are pre-selected, and contracts negotiated and agreed; large projects are split into sub-projects and then put together again, layouted, edited, checked and validated in yet another place. Numerous people and programs handle different parts of the work.

This has significant consequences. It means we have to revise our individualistic concept of the 'translator': a finished translation can be the work of a whole group of actors, e.g., a translation manager, a translation memory, a freelancer, a network of optical fibre cables and a layouter (cf. the cockpit in Hutchins 1995b). But we also have to recognise the defining role played by translation managers (and artefacts/technologies) as the mediators and coordinators of a major part of the complex network: in our case study, they are anything but just a clearing house for translation orders - they play a decisive role in the organisation and completion of the work. In this respect, the case study also offers quasi-empirical confirmation of Latour's and Leontiev's emphasis on tool mediation of cognition and strengthens the notions of collective, distributed problem solving, thus rendering the emergence of highly intertwined sociocognitive network dynamics visible. In other words, it confirms two central hypotheses in situated cognition, ANT and AT in both selected aspects with regard to translation studies as well: firstly that artefacts are indispensable for modern workers and collectives, and secondly that sociocognitive and sociotechnological links and amalgamation are on the rise. Seen from the opposite perspective, these phenomena can only be adequately interpreted and understood in the evolution of translation culture through the broad perspective used in this analysis. 


\section{Conclusions}

Despite the fairly high level of current empirical process research in translation studies, relatively little is yet known about the real genesis of translation cultures and translations in practice. One reason for this is certainly the fact that process research has concentrated on observing students and professional translators in situations that are planned and controlled by the researchers (a necessity when using controlled experiments as the methodological framework of research). Some aspects of the translation process can be studied by observing the translation of an individual text selected by the research team in a room at a university; others can only be studied by observing the handling and completion of a real translation project in a real working environment.

Many more case and multiple longitudinal case studies are therefore needed to bring research into translation into a historical and broader network perspective. In doing so, the methodological framework can be applied in various constellations of action, like the different working situations of freelancers and translation departments in the private and public sector. In this time of great change, it would be particularly beneficial to find out how translators and other participants in the translation process decide, negotiate and justify their decisions (see also Buzelin 2005, 215).

Based on our retrospective analysis of our case study, we have highlighted some methodological findings related to the conceptual frameworks of the situated cognition, ANT and AT approaches. The essential advantage of ANT proved once again to lie in its ability to describe and help understand socio-technical assemblies - the interdependencies and interactions between people and things. Similarly, AT allowed us to describe the tool-mediated aspect of human activities and extend the scope of analysis from individuals and systems/texts to subjects in the social and material world. If artefacts of translatory action are modelled as independent elements, the interdependence between the cognitive translation processes and the artefacts becomes visible. However, this indispensability of socio-technological links and the dynamics of the network also pose key methodological challenges that should be tackled in future empirical studies of the cognitive aspects of translation.

While situative embedding in the roles, positions and dependencies in a network and a translation culture plays an important role, it still does not determine the behaviour of the actors. Nonetheless, the ability to sufficiently understand the dynamics of such a network is definitely part of translation expertise. Far more empirical research is needed before we can actually claim to understand the dynamics of translation culture - or even teach such processes and concepts in translation studies institutes. Establishing our own networks with various neighbouring 
theories and academic cultures (such as, for example, situated cognition, ANT and AT) could add valuable new insights to and takes on our own research.

\section{References}

Abdallah, Kristiina. 2011. "Quality Problems in AVT Production Networks: Reconstructing an Actor-Network in the Subtitling Industry." In Audiovisual Translation in Close-up: Practical and Theoretical Approaches, ed. by Adriana Serban, Anna Matamala, and Jean-Marc Lavaur, 173-186. Bern: Peter Lang.

Bogic, Anna. 2010. "Uncovering the Hidden Actors with the Help of Latour: The 'Making' of the Second Sex." MonTI 2: 173-192. DOI: 10.6035/MonTI.2010.2.9

Buzelin, Hélène. 2005. "Unexpected Allies: How Latour's Network Theory Could Complement Bourdieusian Analyses in Translation Studies." The Translator 11 (2): 193-218.

DOI: $10.1080 / 13556509.2005 .10799198$

Bourdieu, Pierre. 2001. Science de la science et réflexivité. Paris: Éditions Raisons d'Agir.

Callon, Michel. 1986. "Some Elements of a Sociology of Translation: Domestication of the Scallops and the Fishermen of St Brieuc Bay." In Power, Action and Belief: A New Sociology of Knowledge, ed. by John Law, 196-233. London: Routledge \& Kegan Paul.

Clark, Andy. 1997. Being there. Putting Brain, Body, and World Together Again. Cambridge, MA: MIT Press.

Clark, Andy. 2008. Supersizing the Mind: Embodiment, Action, and Cognitive Extension. Oxford: Oxford University Press.

Clark, Andy, and David J. Chalmers. 1998. “The Extended Mind.” Analysis 58 (1): 7-19. DOI: 10.1093 /analys/58.1.7

Donmoyer, Robert. 2000. "Generalizability and the Single-Case Study." In Case Study Method: Key Issues, Key Texts, ed. by Roger Gomm, Martyn Hammersley, and Peter Foster, 45-68. Thousand Oaks, CA: Sage.

Engeström, Yrjö. 1990. Learning, Working, and Imagining: Twelve Studies in Activity Theory. Helsinki: OrientaKonsultit Oy.

Holz-Mänttäri, Justa. 1993. “Textdesign - verantwortlich und gehirngerecht." In Traducere Navem, ed. by Justa Holz-Mänttäri, and Christiane Nord, 301-320. Tampere: Tampereen yliopisto.

Hutchins, Edwin. 1995a. Cognition in the Wild. Cambridge: The MIT Press.

Hutchins, Edwin. 1995b. "How a Cockpit Remembers its Speeds." Cognitive Science 19: 265-288. DOI: $10.1207 / \mathrm{s} 15516709 \operatorname{cog} 1903 \_1$

Kaptelinin, Victor, and Bonnie A. Nardi. 2006. Acting with Technology. Activity Theory and Interaction Design. Cambridge, MA: MIT Press. DOI: 10.5210/fm.v12i4.1772

Kuutti, Kari. 1991a. "Activity Theory and its Applications in Information Systems Research and Design." In Information Systems Research Arena of the 90's, ed. by Hans-Erik Nissen, HeinzKarl Klein, and Rudy A. Hirschheim, 529-550. Amsterdam: North-Holland.

Kuutti, Kari. 1991b. “The Concept of Activity as a Basic Unit for CSCW Research.” In Proceedings of the 2nd ECSCW, 1991, ed. by Liam J. Bannon, Mike Robinson, and Kjeld Schmidt, 249264. Amsterdam: Kluwer.

Latour, Bruno. 1986. "Visualization and Cognition." Knowledge and Society 6: 1-40. 
Latour, Bruno. 2005. Reassembling the Social: An Introduction to Actor-Network Theory. Oxford: Oxford University Press.

Leontiev, Aleksej N. 1978/1987. Activity, Consciousness, and Personality. Translated by Marie J. Hall. Englewood Cliffs, NJ: PrenticeHall.

Martín de León, Celia. 2008. “Translation in the Wild: Traductología y cognicíon situada.” In La traducción del future: mediación lingüística y cultural en el siglo XXI. Vol II. La traducción y su entorno, ed. by Luis Pegenaute, Janet DeCesaris, Mercè Tricás, and Elisenda Bernal, 55-64. Barcelona: PPU.

Menary, Richard (ed). 2010. The Extended Mind. Cambridge, MA: The MIT Press. DOI: $10.4135 / 9781452257044 . n 124$

Oyserman, Daphna. 2011. "Culture as Situated Cognition: Cultural Mindsets, Cultural Fluency, and Meaning Making." European Review of Social Psychology 22 (1): 164-214.

DOI: $10.1080 / 10463283.2011 .627187$

Prunč, Erich. 2007. “Zur Konstruktion von Translationskulturen.” In Translationskultur: Ein innovatives und produktives Konzept, ed. by Larisa Schippel, 19-41. Berlin: Frank \& Timme.

Risku, Hanna. 2010. "Lotsen im soziokulturellen Luftraum - TranslatorInnen im Tower transkultureller Fachkommunikation.” In Translationskultur revisited. Festschrift für Erich Prunč, ed. by Nadja Grbič, Gernot Hebenstreit, Gisella Vorderobermeier, and Michaela Wolf, 173189. Tübingen: Stauffenburg.

Risku, Hanna, Nicole Rossmanith, Andreas Reichelt, and Lukas Zenk. 2013. "Translation in the Network Economy: A Follow-up Study." In Tracks and Treks in Translation Studies, ed. by Catherine Way, Sonia Vandepitte, Reine Meylaerts, and Magdalena Bartlomiejczyk, 29-48. Amsterdam: John Benjamins. DOI: 10.1075/btl.108.02ris

Schäffner, Christina. 1997. "From 'Good' to 'Functionally Appropriate': Assessing Translation Quality." Current Issues in Language and Society 4 (1): 1-5. DOI: 10.1080/13520529709615476

Simeoni, Daniel. 1998. “The Pivotal Status of the Translator's Habitus.” Target 10 (1): 1-39. DOI: $10.1075 /$ target.10.1.02sim

Snell-Hornby, Mary, Zuzana Jettmarová, and Klaus Kaindl(eds). 1997. Translation as Intercultural Communication. Selected Papers from the EST Congress, Prague 1995. Amsterdam: John Benjamins. DOI: 10.1075/btl.20

Sperber, Dan, and Lawrence Hirschfeld. 1999. "Culture, Cognition, and Evolution." In MIT Encyclopedia of the Cognitive Sciences, ed. by Robert Wilson, and Frank Keil, cxi-cxxxii. Cambridge, MA: MIT Press.

Susam-Sarajeva, Şebnem. 2009. “The Case Study Research Method in Translation Studies.” The Interpreter and Translator Trainer 3 (1): 37-56. DOI: 10.1080/1750399X.2009.10798780

Vorderobermeier, Gisella. 2008. "Migration als Übersetzung: Versuch einer Annäherung aus soziokognitiver Sicht." In "Meine Sprache grenzt mich ab...” Transkulturalität und kulturelle Übersetzung in Kontext von Migration, ed. by Gisella Vorderobermeier, and Michaela Wolf, 37-50. Vienna: LIT. 



\title{
Towards a new linguistic-cognitive orientation in translation studies
}

\author{
Juliane House \\ University of Hamburg, Germany
}

A new linguistic-cognitive orientation in translation studies is important today because it can complement the current strong wave of socially and culturally oriented research into and around translation. For balance, it is also necessary and insightful to describe and explain how strategies of comprehending, decisionmaking and re-verbalisation come about in a translator's bilingual mind. In this chapter I sketch some ideas about such a new linguistic-cognitive approach. I first review introspective and retrospective studies and behavioural experiments. Secondly, I assess the value of neuro-linguistic studies for translation. Thirdly, I suggest a new combination of a translation theory and a neuro-functional theory of bilingualism.

Keywords: introspection, consciousness, neuro-linguistic studies, neurolinguistic theory of bilingualism, systemic-functional translation theory, pragmatics, overt and covert translation

\section{A plea for a new linguistic-cognitive orientation in translation studies}

Given the so-called 'turns' in translation studies, I will make a plea for a new linguistic-cognitive orientation. I think this is necessary, because the recent 'turns' have resulted in a predominance of cultural, social, ideological and personal concerns focusing on 'translation at large': the reasons for, and the effects of translation; the needs for, and the means of 'intervention' or 'resistance'; and the moral, ethical, social and political responsibility of translators and their 'visibility. True, there are scholars who have maintained an interest in linguistic matters to this day (e.g. Koller 2011; Malmkjaer 2011a; House 2012 and the late Peter Newmark's many publications). Moreover, there are translation scholars who have had a consistent interest in both linguistic and socio-cultural matters (e.g. Baker 2006; 2011), and there is a research strand - to which I turn below - that has studied 
the translation process since the eighties. However, what is missing is a combined linguistic-cognitive translation theory. This chapter tries to make a modest start towards proposing such a symbiosis.

\section{Rationale for a new linguistic-cognitive orientation}

One reason for suggesting a new linguistic-cognitive orientation is my belief that translation is above all an activity involving language and its cognitive basis. A preoccupation with external social, cultural, personal, historical, etc. factors impinging on translation 'from the outside' (cf. Tymoczko 2007) seems therefore to miss the point about the essence of translation. The widespread view today (cf. Gentzler 2008; Prunč 2011) of translation as an art, coupled with a cult of individual translators, their creativity, influence, status, moral stance, ideological 'positioning' and so on, all stress translation as a translator's new creation. Influential neo-hermeneutic, constructivist and effect-oriented approaches to translation have long propagated an anti-equivalence position. Equivalence-bashing went along with a de-throning of the original and a consequent enthronisation of translators as authors in their own right. In this view, translation is often regarded as a kind of manipulation (cf. Hermans 1985; Stolze 2003; Shamma 2009; and see Reiss and Vermeer's 1984 Skopos theory, which embodies a general licence for manipulation given its maxim of 'the end justifies the means').

I would argue against a view of translation as an individual's art of interpretation by pointing to what Susan Sontag wrote in her famous volume Against Interpretation (1961, 3), where she attacked "the cult of interpretation" as a philistine refusal to leave a text alone. While Sontag refers to literature, her stance on interpretation is also relevant for arguing against an excessive role of subjective interpretation in translation. The function of text analysis for translation is, in my opinion, to show how a text is what it is, that it is what it is, rather than to be pre-occupied with what it means to a reader. This idea harks back to Benjamin's (1923/1992) seminal ideas about interpretation and his implicit prioritization of the text over the individual translator.

Another related, popular idea is linking translation with a translator's 'intention' (Prunč 2011). Since all text production is determined by interests, a translation should reflect the intentions of translators. The illusion of 'interestless intentions' would lead straightaway to a deplorable personal, political and socio-cultural 'invisibility'. However, is not translators' 'visibility' always possible through insertions of prefaces, pre- and postscripts, footnotes, explicit mentioning of the translator's name in the text and so on? 
It is against such a - to my mind - exaggerated concern with the subjectivepersonal in translation that I believe a shift is needed to a focus on both language/ text (the linguistic focus) and on what happens in translators' minds when they are translating (the linguistic-cognitive focus). What is needed is a theoretically based description and explanation of how strategies of comprehending, problem solving and decision making with reference to the texts that translators handle come about in their bilingual minds. Of course, such a focus does not need to be at the expense of the socio-cultural: it has long been recognised that socio-culturally shared knowledge as linguistic-cognitive representations in the form of schemata, scripts, plans, constructions and routines result from conventionalisation processes in a particular culture via the medium of language (cf. Sperber 1996; Cook and Bassetti 2011). This recognition was already influencing translation studies in the 1990s (Wilss 1996). But this early linguistic-cognitive orientation was soon eclipsed by the rise of another paradigm: translation process research. I will not describe this research field in any detail here, since this has been done more competently in many recent works (Göpferich 2008; Göpferich and Jääskeläinen 2009; Jääskeläinen 2011). What I want to do in what follows is cast a critical look at such process-related translation research.

\section{Introspective and retrospective translation process studies: how valid and reliable are their outcomes?}

Introspective and retrospective studies, frequently involving monologic or sometimes dialogic tasks as well as rating and other decision-related tasks have been a very productive research paradigm since their inception in the 1980s (for early work see Krings 1986; House and Blum-Kulka 1986; House 1988). The fundamental question underlying all introspective and retrospective translation studies is that persons involved in the act of translating have substantial control over their mental processes, and that these processes are to a large extent accessible to them (i.e. open to their conscious inspection and verbalisation). It is, however, far from clear that this assumption is valid. Even more important from the point of research methodology is the fact that, at present, it is not clear that this assumption CAN be confirmed or falsified.

There seem to be at least five largely unresolved questions with regard to translation-related introspective and retrospective research methodology:

1. Is what ends up being verbally expressed in thinking aloud sessions really identical with underlying cognitive processes? 
2. Exactly which cognitive processes are accessible to verbalisation and which are not; that is, how can one differentiate between metacognitive monitoring and reflective (declarative) behaviour on the one hand and routinised (procedural) behaviour on the other hand?

3. Does the fact that translators are asked to verbalise their thoughts while they are engaged in translating change those cognitive processes that are (normally) involved in translation? In other words, are translators engaged in introspection sessions subject to the so-called 'observer's paradox'? To be fair, this question has been addressed by translation process researchers, such as Jakobsen (2003), who pointed to a slow-down effect of the 'double-burden' of translating and talking about it. Jakobsen also noted that translation units tend to become smaller when subjects are asked to verbalise while translating.

4. What happens to those parts of (often expert) translators' activity that are highly, if not entirely, routinised and automatised and are thus by definition not open to reflection? (cf. Königs 1986, who distinguished an automatic, adhoc block from a 'rest' block of cognitive translation activity).

5. With regard to retrospective translation-related research: how can data from ex post facto interviews or questionnaires access translation processes given working memory constraints and given the pressure felt by subjects to provide data that will satisfy the researcher? Is it not likely that subjects will make meta-statements about what they think they had thought?

These five questions, and possibly more, touch upon one of the most important and most controversial issues in contemporary cognitive science: the nature of consciousness. Much recent neuroscience literature stresses in fact the importance of the non-conscious - a depressing finding for translation process research (cf. Suhler and Churchland 2009; Nosek et al. 2011). Others, however, stress the need for a comprehensive theory of consciousness that goes beyond an exclusive focus on (inaccessible) representations trying to explain "how those representations are experienced and accessed by the multiple functions constituting an observer" (Cohen and Dennett 2011,363). In other words, for a viable theory of consciousness, the relation between function and experience needs to be unravelled.

Fortunately, there is an increasing awareness of the critical methodological issues in translation process research mentioned above. Thus in a paper with the promising title: "Back to Basics: Designing a Study to Determine the Validity and Reliability of Verbal Report Data on Translation Processes", Jääskeläinen (2011) very sensibly points to the need for a systematic methodological study on the use of verbal report data, a study that would take into account the specific nature of translation tasks and incorporate contrastive analyses of the language pairs involved in the translation at hand. This is an encouraging sign for the discipline. 


\section{Behavioural experiments on the translation process: how valid, reliable and insightful are their outcomes?}

Given the type of discontent with attempts to look into the translator's 'black box' in introspective and retrospective translation process research described above, translation scholars have now tried to remedy the situation. They have come up with more controlled behavioural experiments designed to avoid making claims about the 'black box' and directly trace linear and non-linear translational steps and phases, measuring the temporal progress or delay, the types and numbers of revisions undertaken by the translator, the (measurable) effort expended, the nature and number of attention foci and attention shifts as well as the frequency and kind of emotional stress responses shown by the translator while translating. This ambitious agenda was made possible through recent, mostly computer-related technological progress such that experiments using keystroke logging, screen recording, eye tracking and various physiological measures could be undertaken. A recent overview of this line of behavioural translation-related research that often neatly combines various tools (e.g. keystroke logging and eye tracking), and also increasingly triangulates thinking aloud data with experimental ones is provided in Shreve and Angelone (2010) and O'Brien (2011). O'Brien (2011, 11) makes the important general point that much of this type of translation process research regularly displays great individual variation, which, she claims, is only to be expected given the fact that we are here dealing with individual human beings. She points to Hansen's (2010) proposal of going beyond those predominantly quantitative data elicited via keystroke logging, eye tracking, etc. by attempting a more integrative take on the translation process involving a translator's 'life story'. I beg to disagree with this idea, because such an undertaking would lead us to the same personalisation in translation studies which I criticised above. If we aim at having translation studies recognized as a science, which I believe we should, if we want to be respected as a serious discipline, then we have to aim at generalisations, for which we need first and foremost transdisciplinary cooperation with cognitive and bilingual research coupled with a good theory. I will take up this idea below. Now I will first ask two critical questions with regard to the validity and reliability of the behavioural measures used in experimental behavioural translation process research:

1. Can measurements of observable behaviour (as provided in keystroke logging, eye tracking, etc.) inform us about cognitive processes that occur in a translator's mind?

2. Can measurements of observable behaviour explain the nature of cognitive representations of the two languages, throw light on a translator's meta-linguistic 
and linguistic-contrastive knowledge, comprehension, transfer and reconstitution processes emerging in translation procedures?

I would answer both questions with: 'not really'. What such experiments CAN and DO measure is exactly what they set out to measure: observable behaviour, no more and no less. This is NOT to belittle their worth - far from it. All I am arguing here is that the results of such behavioural experiments should not be taken as indications of cognitive processes in the minds of translators. Rather they should be seen as interesting hypotheses. If such experiments are combined with theoretical models that incorporate features of semantic representation and of processing, they may pave the way towards abandoning any clear-cut distinction between product and process in favour of a more holistic and unitary perspective (cf. Halverson 2009). It is necessary, however, to always clearly differentiate between cognitive processes and the underlying neural correlates. The number of fixations, gaze time, pause lengths and incidences of self-corrections examined in keystroke-logging and eye-tracking experiments cannot be taken to be clear evidence of the involvement of certain neurological substrates. Rather, they are likely to indicate certain translation difficulties (cf. Dragsted 2012) and attendant decision processes, and these may involve certain neural networks more than others. Still, the crux is that the involvement of neural network $x$ cannot tell us exactly which processes are connected with neural network $\mathrm{x}$. In other words, the connection between cognitive processes and underlying neural connections is still a mystery today.

Recently, many translation scholars have set their hopes on another new, exciting research strand: bilingual neuro-imaging studies. In the following, I will look at how promising they are for the field of translation studies.

\section{Bilingual neuro-imaging studies: how useful and relevant are they for translation studies?}

Can neuro-imaging studies give us 'a direct window' onto the translator's 'black box', on what goes on in a translator's mind, finally providing us with a solution to Krings' question in 1986 "What happens in translators' heads?" First of all, the value of the findings of such studies is controversial (cf. Aue et al. 2009), not least because they are obviously crucially dependent on the type of task used. With the exception of some rare recent use of isolated sentences, functional magnetic resonance imaging (fMRI), positron emission tomography (PET) and event-related potential (ERP) studies are word-based (cf. de Groot 1997; Price et al 1999; Klein et al 2006; Hernandez 2009). Translation, however, is essentially text-based. 
Any application of neuro-imaging experimental research to translation thus faces the dilemma that translation research is essentially interested in less controllable, larger and more 'messy' units.

So we can conclude that these studies essentially lack ecological validity due to their inherent task artificiality. Why this should be so can be answered with reference to another neuroscientist, Michel Paradis, who stated: "The use of any task other than the natural use of language (including natural switching and mixing) has the same consequence as using single words: the task does not tap the normal automatic processes that sustain the natural use of language including the contribution of pragmatics and its neural underpinnings" (2009, 157-158).

Over two-thirds of neuro-imaging studies on laterality and language switching and mixing use single words as stimuli, for instance, in picture-naming experiments where subjects are asked to switch on command (but see Abutalebi 2008 for an exception to the use of single words in such experiments). However, as Paradis $(2009,160)$ has pointed out, brain activity crucially differs for language use in natural situations and in language use 'on cue', and, most importantly, these situations correspond to opposite types of processes. Indeed, single words are very different from the rest of language. They are part of the (conscious) vocabulary of a language, not part of the lexicon. The latter includes morphosyntactic properties and is integrated into each language subsystem's neural network in the bilingual brain. Single-word stimuli are explicitly known form-meaning associations subserved by declarative memory, while procedural memory underlies normal, natural language use. Each memory system relies on distinct neuro-functional structures. And normal, natural language use also critically involves cortical areas of the brain's right hemisphere to process the pragmatic aspects of utterances this, however, is irrelevant in processing single words that are used out of context.

Another problem with neuro-imaging data that needs to be addressed relates to the nature of the evidence from neuro-imaging data: blood flow and other hemo-dynamic responses routinely provided in such data cannot be taken to be direct measures of neuronal activity.

Further, and this is a serious methodological drawback indeed, most neuroimaging studies have not been replicated. Many reported neurological activations are strongly task-dependent and rely on a particular technique employed, so that replication is difficult. And it is this task and technique dependence which suggests that the reported activations in the brain are indicative of the particular task and technique employed rather than being indicative of language representation, processing and switching per se.

Given these shortcomings, it is advisable to first look for a theory with enough descriptive and explanatory potential before expecting enlightenment from experimental neuro-imaging studies, whose usefulness for translation studies is, at the 
present time, not clear at all. An overly optimistic view of neuro-imaging studies like the one previously expressed by the present author (House 2011) is therefore not warranted at the present time.

\section{A neuro-linguistic theory of the functioning of two languages in the brain}

The neuroscientist Paradis has set up his own neuro-linguistic theory of the bilingual mind. His model (Paradis 2004, 227) depicting the neuro-functional and linguistic-cognitive system of the bilingual mind is reproduced in Figure 1.

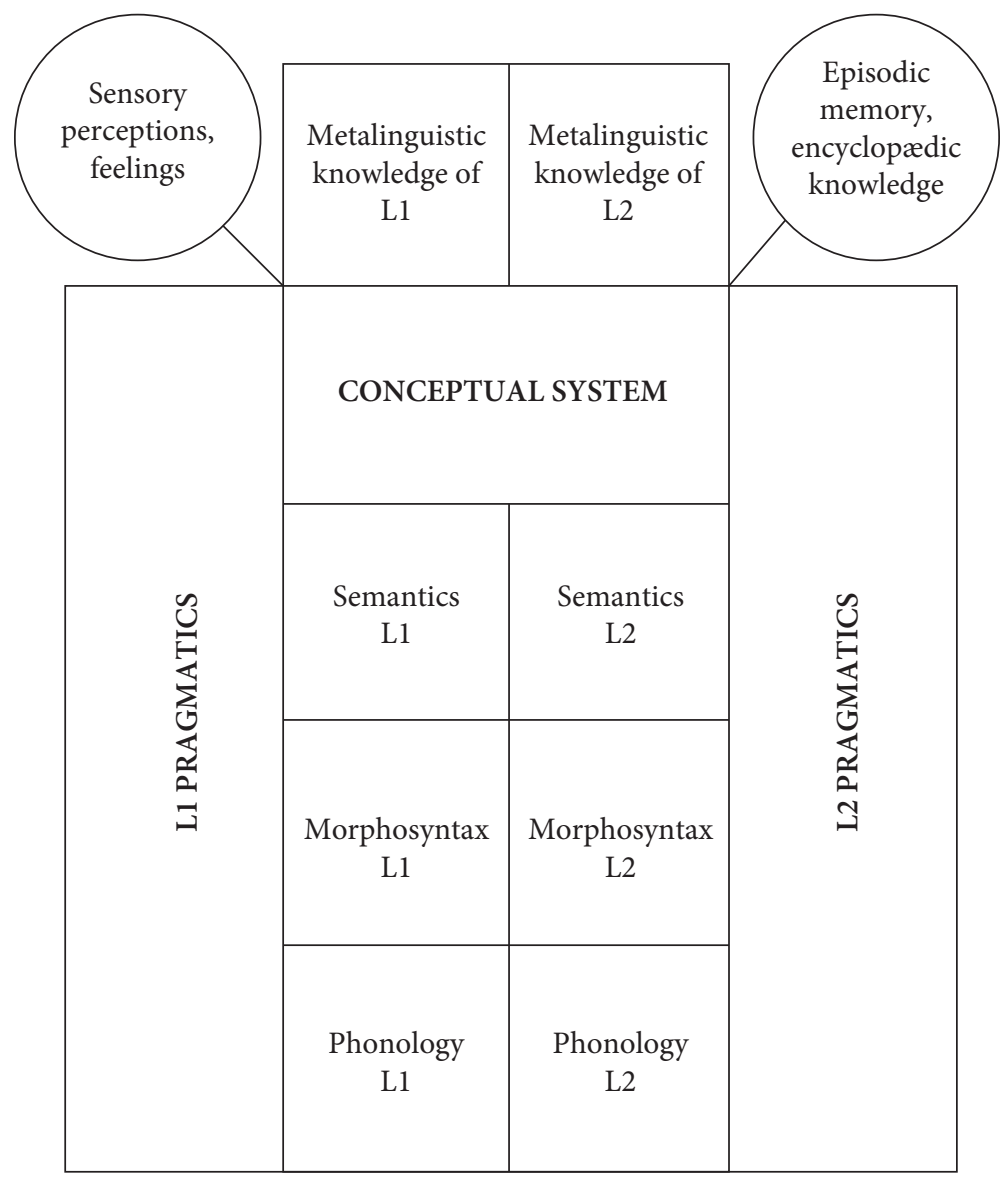

Figure 1. Paradis' model (reproduced from Paradis 2004, 227, with the permission of John Benjamins Publishing Co.) 
The model features different levels for explicit metalinguistic knowledge of a bilingual's two languages L1 and L2, sensory perceptions, feelings, episodic memory and encyclopaedic knowledge, a joint conceptual system and different languagespecific levels of semantics, morphosyntax and phonology. Conceptual mental representations are independent of language. In translational L1 and L2 contact situations, the degree of overlap depends on their relative typological closeness.

Paradis' model emphasises the need "to distinguish between representation and control, between what is represented and how it is represented, between what is represented and how it is accessed, and between what is represented in each language and how these language representations are organised in the brain into systems or subsystems" (2004, 230-231).

In Paradis' model, L1 and L2 pragmatics encompass and feed into both the conceptual system and the different language levels. Implicit linguistic competence and metalinguistic knowledge are independent systems. Only the use of metalinguistic knowledge is consciously controlled. The use of implicit competence is automatic, devoid of conscious effort, awareness of the process involved or attention focussed on the task on hand. Languages are represented as neuro-functional subsystems of the language system (the implicit linguistic competence), which is a component of the verbal communication system that, in addition to the language system, contains metalinguistic knowledge, pragmatic ability and motivation. This verbal communication system is connected to the cognitive system where intentions to communicate a message are formulated or messages are received and interpreted according to the lexico-grammatical constraints of L1 and L2 that activate the relevant concepts and depend on pragmatic context-dependent inferences. The intention to communicate triggers the verbalisation of the message formulated in the cognitive conceptual system. The implicit linguistic competence ('the grammar') constrains the encoding of the message, and the pragmatics component makes selections in terms of styles, registers, discourse norms, speech act directness, politeness, etc.

Paradis suggests that bilinguals (including translators) have two subsets of neuronal connections, one for each language, and these are activated or inhibited (for instance in the process of translation) independently. But there is also one larger set from which they can draw items of either language at any one time. All selections are automatic (i.e., unconsciously driven by activation levels). With specific reference to translation, Paradis proposes the operation of two distinct translation strategies:

1. A strategy of translating via the conceptual system, involving processes of linguistic decoding (comprehension) of source-text material plus encoding (production) of target-text material. 
2. Direct transcoding by automatic application of rules, which involves moving directly from linguistic items in the source language to equivalent items in the target language. In other words, source language forms immediately trigger target language forms, thus bypassing conceptual-semantic processing (cf. here also Göpferich's (2008) “translation-routine activation competence”).

Paradis' theory is relevant for translation in that he presents an explanation for the representation modi of two languages as keys to essential translation processes of decoding, comprehending, transferring, re-assembling and re-verbalising. Of particular significance in his model is, I believe, the overriding importance he assigns to the L1 and L2 pragmatics components which impact on the conceptual system and on the other linguistic levels. With regard to the separate shared conceptual system, the model can explain that expert translators often do not need to access it as they move directly from the source to the target language (cf. Königs 1986 and Tirkkonen-Condit 2004 for empirical evidence).

The importance assigned by Paradis to the pragmatics component suggests the possibility of combining his model of the bilingual (translator's) brain with a functional-pragmatic translation theory of linguistic text analysis, translation and translation evaluation (House 1977; 1997; 2009). This theory is designed to explicate how pragmatic, textual and lexico-grammatical meanings in an original text are re-constituted in a different context, with the translated text being either a functionally equivalent re-constitution of a source text or a complete contextual adaptation to the new L2 environment. The model provides a principled procedure for a comprehensive linguistic-textual analysis and, in the case of its use in evaluation, for a comparison of the textual profiles of source and target texts. It also integrates relevant contrastive linguistic and pragmatic research.

Two fundamental operations of translation are hypothesised in this model: overt translation and covert translation. They are defined as outcomes of different types of re-contextualisation procedures with qualitatively different cognitive demands on the translator: overt translation is psycho-linguistically and cognitively complex, covert translation is simple. In overt translation, the addressees and recipients of the translation are quite 'overtly' not directly addressed. While embedded in its new target-culture context, the translation signals at the same time its 'foreign origin'. An example would be a speech given by a prominent representative of the L1 linguaculture delivered at a specific time and place. The translator's work in translating this speech is here important and visible. The translation can be said to be a case of 'language mention', resembling a quote. The addressees and recipients of the translation are meant to appreciate the source text in a new frame and a new discourse world. The pragmatics of the source text and the target text are mentally co-activated, and this is why overt translation can be considered 
psycho-linguistically and cognitively complex. 'Real' functional equivalence cannot be achieved, and is also not aimed at - only a kind of second-level functional equivalence is possible.

Covert translation, on the other hand, enjoys the status of an original text in the target linguacultural context. For the recipient of the translation in the target linguaculture, it is not marked pragmatically as a translation at all. It is a case of 'language use', a functionally equivalent speech event created by the translator. There is no co-activation of the pragmatics of the source text and the target text in the recipient's mind, and it is this absence of mental co-activation which explains why covert translation can be said to be a psycho-linguistically and cognitively simple act. Covert translation often involves massive intervention on the levels of language/text, register and genre. And in order to achieve the necessary functional equivalence, the translator needs to make allowance for the target text's pragmatics component. This can be done via the use of a so-called 'cultural filter', a construct capturing differences in source and target text's addressees' linguaculturally determined conventions and expectation norms. Cultural filtering should ideally be based on empirical cross-linguistic and cross-cultural research to guide and explain translators' choices. Examples of such research are the studies conducted by the present author over many years on English-German discourse norms in oral and written texts in many genres. They point to differences in preferences for explicitation, directness, interpersonal focus and use of verbal routines (cf. House 2006a, b). With regard to other language pairs, there is a deplorable lack of systematic contrastive pragmatic work on register and genre variation, which renders a solid theoretical underpinning of translation studies in this respect next to impossible. What is clearly needed here is a combination of qualitative, quantitative, exemplarand corpus-based as well as experimental cross-cultural research (for promising suggestions of such a combination, see Halverson 2010 and Alves et al. 2010).

Returning to Paradis' $(2004 ; 2009)$ model: how important is it for linguisticcognitive translation studies, and might it be combined with a functional translation theory such as, for instance, the one described above? Paradis' model is, I think, highly relevant for translation studies (cf. Malmkjaer 2011b, who also makes this point), and it may be combined with an existing translation model (House 1997; 2009) for the following reasons.

The importance of the L1 and L2 pragmatics components in Paradis' model provides support for the assumptions underlying the functional pragmatic translation theory described above, in particular with reference to

a. the concept of the cultural filter in covert translation with its hypothesised complete switch to L2 pragmatic norms

b. the hypothesised co-activation of L1 and L2 pragmatics components in overt translation. 
Paradis' model accounts for the claim in the functional-pragmatic translation theory described above that overt translation is psycho-linguistically more complex due to an activation of a wider range of neuronal networks - across two pragmatics-cum-linguistics representational networks (cf. Figure 1) in the translation process. It also accounts for the claim that covert translation is psycho-linguistically simple: here only one pragmatics-cum-linguistics representational network - that of the $\mathrm{L} 2$ - is activated in the process of translation. At the present time this is a hypothesis to be tested empirically.

\section{Conclusion}

For a new linguistic-cognitive orientation in translation studies that may emanate from a critical look at research involving intro- and retrospection, behavioural experiments and neuro-imaging studies, a fresh attempt at theorising might be a fruitful beginning.

I have suggested that as a first step towards a valid and reliable approach to investigating the translation process, one may look for a descriptively and explanatorily adequate neuro-linguistic theory of bilingualism that can be useful for, and compatible with, a theory of translation.

The combination suggested in this chapter is just one possible first attempt at construing a rapprochement between the disciplines of cognitive science and linguistically-cognitively-oriented translation studies. Other more potent examples may be suggested in the course of scientific inquiry, and it may well be that these effectively falsify the rather general suggestions sketched in this chapter.

\section{References}

Abutalebi, Jubin. 2008. "Neural Aspects of Second Language Representation and Language Control." Acta Psychologica 128: 466-478. DOI: 10.1016/j.actpsy.2008.03.014

Alves, Fabio, Adriana Pagano, Stella Neumann, Erich Steiner, and Silvia Hansen-Schirra. 2010. "Translation Units and Grammatical Shifts: Towards an Integration of Product- and Process-Based Translation Research.” In Translation and Cognition, ed. by Gregory Shreve, and Erik Angelone, 109-142. Amsterdam: John Benjamins. DOI: 10.1075/ata.xv.07alv

Aue, Tatjana, Leah Lavelle, and John Cacioppo. 2009. "Great Expectations: What can fMRI Research Tell Us about Psychological Phenomena?” International Journal of Psychophysiology 73 (1): 10-16. DOI: 10.1016/j.ijpsycho.2008.12.017

Baker, Mona. 2006. Translation and Conflict: A Narrative Account. London: Routledge.

Baker, Mona. 2011. In Other Words. A Course Book on Translation, 2nd ed. London: Routledge. Benjamin, Walter. 1923/1992. “The Task of the Translator." English Translation by Harry Zohn. In Theories of Translation. An Anthology of Essays from Dryden to Derrida, ed. by Rainer Schulte, and John Biguenet, 71-82. Chicago: University of Chicago Press. 
Cohen, Michael, and Daniel Dennett. 2011. “Consciousness cannot be Separated from Function.” Trends in Cognitive Sciences 15 (8): 358-364. DOI: 10.1016/j.tics.2011.06.008

Cook, Vivian, and Benedetta Bassetti (eds). 2011. Language and Bilingual Cognition. New York: Psychology Press.

Dragsted, Barbara. 2012. "Indicators of Difficulty in Translation: Correlating Product and Process Data." Across Languages and Cultures 13 (1): 81-98. DOI: 10.1556/Acr.13.2012.1.5 Gentzler, Edwin. 2008. Translation and Identity in the Americas. London: Routledge.

Göpferich, Susanne. 2008. Translationsprozessforschung: Stand - Methoden - Perspektiven. Tübingen: Narr.

Göpferich, Susanne, and Riitta Jääskeläinen (eds). 2009. "Process Research into the Development of Translation Competence: Where are We, and Where do We Need to Go?" Across Languages and Cultures 10 (2): 169-191. DOI: 10.1556/Acr.10.2009.2.1

de Groot, Annette. 1997. "The Cognitive Study of Translation and Interpretation: Three Approaches." In Cognitive Processes in Translation and Interpreting, ed. by Joseph H. Danks, Gregory Shreve, Stephen Fountain, and Michael McBeath, 25-56. Thousand Oaks: Sage.

Halverson, Sandra. 2009. "Psycholinguistic and Cognitive Approaches." In Routledge Encyclopedia of Translation Studies, 2nd ed., ed. by Mona Baker, and Gabriela Saldanha, 211-216. London: Routledge.

Halverson, Sandra. 2010. "Cognitive Translation Studies: Developments in Theory and Methods." In Translation and Cognition, ed. by Gregory Shreve , and Erik Angelone, 349370. Amsterdam: John Benjamins. DOI: 10.1075/ata.xv.18hal

Hansen, Gyde. 2010. “Integrative Description of Translation Processes." In Translation and Cognition, ed. by Gregory Shreve, and Erik Angelone, 189-212. Amsterdam: John Benjamins. DOI: 10.1075/ata.xv.11han

Hermans, Theo. 1985. The Manipulation of Literature: Studies in Literary Translation. London: Croom Helm.

Hernandez, Arturo. 2009. "Language Switching in the Bilingual Brain: What's Next?” Brain and Language 109: 133-140. DOI: 10.1016/j.bandl.2008.12.005

House, Juliane. 1977. A Model for Translation Quality Assessment, 2nd ed. 1981. Tübingen: Narr. House, Juliane. 1988. “Talking to Oneself or Thinking with Others? On Using Different Thinking Aloud Methods in Translation." Fremdsprachen Lehren und Lernen 17: 84-98.

House, Juliane. 1997. Translation Quality Assessment: A Model Re-Visited. Tübingen: Narr.

House, Juliane. 2006a. "Communicative Styles in English and German." Journal of English Studies 10 (3): 249-267. DOI: 10.1080/13825570600967721

House, Juliane. 2006b. “Text and Context in Translation.” Journal of Pragmatics 38 (3): 338-358. DOI: 10.1016/j.pragma.2005.06.021

House, Juliane. 2009. Translation. Oxford: Oxford University Press.

House, Juliane. 2011. “Translation and Bilingual Cognition.” In Language and Bilingual Cognition, ed. by Vivian Cook, and Benedetta Bassetti, 519-528. New York: Psychology Press.

House, Juliane. 2012. “Translation, Interpreting and Intercultural Communication." In The Routledge Handbook of Language and Intercultural Communication, ed. by Jane Jackson, 495-509. London: Routledge.

House, Juliane, and Shoshana Blum-Kulka (eds). 1986. Interlingual and Intercultural Communication. Tübingen: Narr.

Jääskeläinen, Riitta. 2011. "Back to Basics: Designing a Study to Determine the Validity and Reliability of Verbal Report Data on Translation Processes." In Cognitive Explorations of Translation, ed. by Sharon O’Brien, 15-29. London: Continuum. 
Jakobsen, Arnt Lykke. 2003. "Effects of Think Aloud on Translation Speed, Revision and Segmentation." In Triangulating Translation. Perspectives in Process Oriented Research, ed. by Fabio Alves, 69-95. Amsterdam: John Benjamins. DOI: 10.1075/btl.45.08jak

Klein, Denise, Robert Zatorre, Jen-Kai Chen, Brenda Milner, Joelle Crane, Pascal Belin, and Marc Bouffard. 2006. "Bilingual Brain Organisation: A Functional Magnetic Resonance Adaptation Study." Neuroimage 31: 366-375. DOI: 10.1016/j.neuroimage.2005.12.012

Koller, Werner. 2011. Einführung in die Übersetzungswissenschaft, 8th ed. Tübingen: Francke (UTB).

Königs, Frank. 1986. "Adhoc versus Rest-Block: textuelle Elemente als Auslöser des Übersetzungsprozesses und didaktische Entscheidungshilfen." In Neue Entwicklungen in der Angewandten Linguistik, ed. by Wolfgang Kühlwein, 15-36. Tübingen: Narr.

Krings, Hans. 1986. Was in den Köpfen von Übersetzern vorgeht. Tübingen: Narr.

Malmkjaer, Kirsten. 2011a. "Linguistics and Translation." In Handbook of Translation Studies, Vol. 2, ed. by Yves Gambier, and Luc van Doorslaer, 349-358. Amsterdam: John Benjamins. DOI: $10.7202 / 1018812 \mathrm{ar}$

Malmkjaer, Kirsten. 2011b. "Translation Universals." In The Oxford Handbook of Translation Studies, ed. by Kirsten Malmkjaer, and Kevin Windle, 83-93. Oxford: Oxford University Press. DOI: 10.1093/oxfordhb/9780199239306.001.0001

Nosek, Brian, Carlee Hawkins, and Rebecca Frasier. 2011. "Implicit Social Cognition: from Measures to Mechanisms." Trends in Cognitive Sciences 15 (4): 152-159.

DOI: $10.1016 /$ j.tics.2011.01.005

O’Brien, Sharon (ed). 2011. Cognitive Explorations of Translation. London: Continuum.

Paradis, Michel. 2004. A Neurolinguistic Theory of Bilingualism. Amsterdam: John Benjamins. DOI: $10.1075 /$ sibil.18

Paradis, Michel. 2009. Declarative and Procedural Determinants of Second Languages. Amsterdam: John Benjamins. DOI: 10.1075/sibil.40

Price, Cathy, David Green, and Roswitha von Studnitz. 1999. "A Functional Imaging Study of Translation and Language Switching." Brain 122: 2221-2235.

DOI: 10.1093/brain/122.12.2221

Prunč, Erich. 2011. Entwicklungslinien der Translationswissenschaft. Berlin: Frank \& Timme.

Reiss, Katharina, and Hans Vermeer. 1984. Grundlegung einer allgemeinen Translationstheorie. Tübingen: Niemeyer.

Shamma, Tarek. 2009. Translation and the Manipulation of Difference. Manchester: St. Jerome. Shreve, Gregory, and Erik Angelone (eds). 2010. Translation and Cognition. Amsterdam: John Benjamins. DOI: 10.1075/ata.xv.01shr

Sontag, Susan. 1961. Against Interpretation. New York: Harper \& Row.

Sperber, Daniel. 1996. Culture: A Naturalistic Approach. Oxford: Blackwell.

Stolze, Radegundis. 2003. Hermeneutik und Translation. Tübingen: Narr.

Suhler, Christopher, and Patricia Churchland. 2011. "Control: Conscious and Otherwise." Trends in Cognitive Sciences 15 (8): 341-347. DOI: 10.1016/j.tics.2011.06.002

Tirkkonen-Condit, Sonja. 2004. "Unique Items: Over- or Underrepresented in Translated Language?" In Translation Universals - Do They Exist? ed. by Anna Mauranen, and Pekka Kujamäki, 177-184. Amsterdam: John Benjamins. DOI: 10.1075/btl.48.14tir

Tymoczko, Maria. 2007. Enlarging Translation, Empowering Translators. Manchester: St. Jerome. Wilss, Wolfram. 1996. Knowledge and Skills in Translator Behaviour. Amsterdam: John Benjamins. DOI: 10.1075/btl.15 


\title{
Translation competence
}

\section{Explaining development and stagnation from a dynamic systems perspective}

\author{
Susanne Göpferich \\ Justus Liebig University Giessen, Germany
}

\begin{abstract}
This chapter introduces Dynamic Systems Theory (DST) as a framework for the investigation of translation competence development. After a presentation of the basic concepts and assumptions underlying this theory, results from the longitudinal study TransComp will be discussed against the background of DST. TransComp is a three-year product- and process-oriented longitudinal study of the development of translation competence in 12 students of translation, whose translation products and processes were compared with those of 10 professional translators. The chapter outlines both the difficulties involved in the application of DST to the investigation of translation competence development and the added value that it promises for our understanding of developmental processes in translators, including the ways they can be fostered in translation training.
\end{abstract}

Keywords: translation competence development, Dynamic Systems Theory, DST, expertise, novice-expert paradigm, contrastive analyses, didactical implications, cognitive apprenticeship, translatology

\section{Introduction}

The investigation of translation competence development is a field of research that is still in its infancy. Only about a decade ago, Schäffner and Adab (2000, viii) deplored that there had not yet been "a specific research focus within Translation Studies on how translation competence can be defined and developed". The situation has changed since then. Both individual researchers and research groups have launched projects investigating the development of translation competence (see the overview in Englund-Dimitrova 2005, 14-15; and Göpferich 2008, 168-178). Longitudinal studies in the strictest sense of the term, i.e., of the same individuals at regular intervals during their training and later professional careers, are rare. 
Only such longitudinal studies, however, can provide us with insights into the development of translation competence in its continuity.

Why is it important to investigate the development of translation competence in its continuity? Answers can be provided by Dynamic Systems Theory (DST; Thelen and Smith 1994; Van Gelder 1989). DST has found its way from mathematics into various other fields of research, such as developmental psychology (see Thelen and Smith 1994), second language acquisition (e.g., de Bot, Lowrie, and Verspoor 2007; Verspoor, Lowrie, and van Dijk 2008), and writing skill development (e.g., Verspoor et al. 2004). To the best of my knowledge, however, it has not yet found its way into translation studies.

What are the basic assumptions underlying DST? Competences or skills are envisaged in DST as dynamic systems, i.e., sets of variables that are interconnected and thus interact over time (de Bot et al. 2007, 8). With regard to translation competence, these variables can be regarded as variables for translation sub-competencies that, in their entirety, make up translation competence. These individual sub-competencies may not develop at the same pace, nor will they always develop in a linear manner. Some may stagnate while others continue to develop. Certain sub-systems (i.e., sub-competencies or clusters of sub-competencies) may be precursors of other sub-systems in the developmental process. For a specific subcompetence to start developing, it may be necessary for other sub-competencies to have exceeded a certain threshold value. If we start from my translation competence model (Göpferich 2008, 155; see Figure 1 below), which is an effort model that assumes limited working memory capacity (Gile 1995; 1997), it appears plausible to assume that the value one variable takes has an effect on all the other variables. For example, if one sub-competence reaches an advanced level, which may result in automatized performance of the respective tasks, working memory capacity is released. This capacity then becomes available for the application of other sub-competencies and their development, such as the capacity to make more creative non-obligatory shifts and to consider a wider context, for which there may not have been enough cognitive resources left as long as other sub-processes still needed cognitive effort.

If problem solving is considered an activity that is composed of the application of a certain amount of a) recurrent routine skills in the sense of automatized or proceduralized skills and b) strategic competence, in which the specific measures to apply have to be developed creatively for each individual case, then the difference between novices and experts in the cognitive efforts involved in the solution of a problem can be modelled as the relationship of cognitive resources needed for automatized recurrent skill application to those needed for strategy application. Until at least a certain amount of automatized recurrent skill application 
sets enough cognitive resources free for more cognitively-demanding strategic measures, no development of strategy application can occur.

DST further assumes that, in dynamic systems, repeller states and attractor states exist. These can best be explained by means of the metaphor of a plane surface with holes and bumps in it on which a ball rolls. The ball and its trajectory on the plane represent a specific competence and its development path. On its ways over the plane, the ball is attracted by the holes (attractor states) and repelled by the bumps (repeller states). To get it out of a hole, much energy is needed. Such holes can explain fossilization of certain errors or stages of development, as observed in second-language acquisition, which can only be overcome by intensive training. The development that a particular person undergoes, i.e., the trajectory of the ball, may be highly individual, yet attractor states account for the fact that certain sub-competencies seem to occur in a specific order or at a specific stage of competence development because the corresponding attractor states, in an evolutionary perspective, possess qualities that make the application of cognitive resources settle into an equilibrium at these stages. In DST, the fact that a certain competence stage has been achieved becomes visible by a relative stagnation in the development of the values the various variables that make up the system take at this stage, whereas a move out of the hole, a new competence development burst, shows in a large variability in the set of variables which form the competence system (Thelen and Smith 1994, 97).

As Thelen and Smith $(1994,67)$ admit, the principles of dynamic systems are "extremely general" so that "as a whole the approach may indeed not be empirically verifiable". However, the approach is also extremely powerful and "the principles do generate specific predictions which can, and have been, confirmed in the developmental literature" and in their own work (Thelen and Smith 1994, 69).

What complicates the analysis of the development of translation competence in a DST approach is that dynamic systems are nested, i.e., every system is always part of a larger system. Accordingly, translation competence forms a sub-system of the larger system of communicative competence, although some believe it is the other way around (cf. Figure 1). This nesting or embeddedness makes it difficult to draw a border around the system an investigation focuses on, and drawing such a border always means ignoring factors beyond the border that may have effects relevant to the system in focus.

Another challenge for the investigation of translation competence development in the DST paradigm is to find the variables of translation competence, i.e., the sub-competencies that are highly sensitive to changes and, at the same time, have measurable effects on translation performance as a whole. Here we have to take into account, as another complicating factor, that dynamic systems may develop in a non-linear manner. This means that: 
there is a non-linear relation between the size of an initial perturbation of a system and the effects it may have in the long run. Some minor changes may lead to huge effects, while major perturbations may be absorbed by the system without much change. (de Bot et al. 2007, 8)

Another highly relevant characteristic of dynamic systems that complicates their investigation, however, is that they are constantly changing "through interaction with their environment and through self-organization" (de Bot et al. 2007, 8). With regard to translation competence, change through "interaction with their environment" means that DST also takes into account the social-constructivist and cultural aspects of translation, in the form, for example, of translation norms and collaborative networks in which a translator is involved. Thus, for de Bot et al. (2007, 18-19), DST "provides us with a framework and an instrumentation that allows us to merge the social and the cognitive aspects of SLA [second-language acquisition] and shows how their interaction can lead to development". The same presumably can be said about translation competence development.

The present chapter represents an attempt to (re-)interpret some of the results of the longitudinal study TransComp, ${ }^{1}$ whose experimental design, measuring instruments and procedures of analysis are described in detail in Göpferich (2009; $2010 ; 2011)$ in light of DST. The chapter outlines both the difficulties involved in the application of DST to the investigation of translation competence development and the added value that it promises for our understanding of developmental processes in translators, including the ways they can be fostered in translation training.

\section{What didactically relevant variables is the dynamic system of translation competence composed of, and how can they be 'measured'?}

One prerequisite for a differentiated analysis of the development of translation competence is the knowledge of the variables in which translation products and processes of highly competent translators differ from those of less competent translators at different stages of their training or development. From contrastive studies which investigated translation products and processes of novices as compared with advanced students of translation, bilinguals or professional translators, we know certain variables in which their products and processes differ (EnglundDimitrova 2005, 14-15; Göpferich 2008, 168-178). What we do not know from these studies, however, is how these variables develop over time and in relation to

1. For TransComp (project No. P20909-G03), funding is acknowledged from the Austrian Science Fund (FWF). 
each other, because the participants whose products and processes were analyzed at different competence stages (novice vs. expert) were not the same.

The problem can be solved, at least to some extent, by means of longitudinal studies in the strictest sense. One of the few studies to date that fulfils these requirements is the longitudinal study TransComp (Göpferich 2009). This study investigated the development of translation products and processes of 12 students of translation over the 3-year period of their bachelor's program using think-aloud, keystroke logging and screen recording as its main methods of data collection and compared their products and processes to those of 10 professional translators with at least 10 years of professional experience in translation and/or interpreting (for details, see Göpferich 2009; for the entire corpus including the source texts, see Göpferich, Bayer-Hohenwarter, and Stigler 2011). In TransComp, translation competence was modelled as depicted in Figure 1.

TransComp focused on the development of those sub-competencies in this model that were assumed to be specific to professional translation competence:

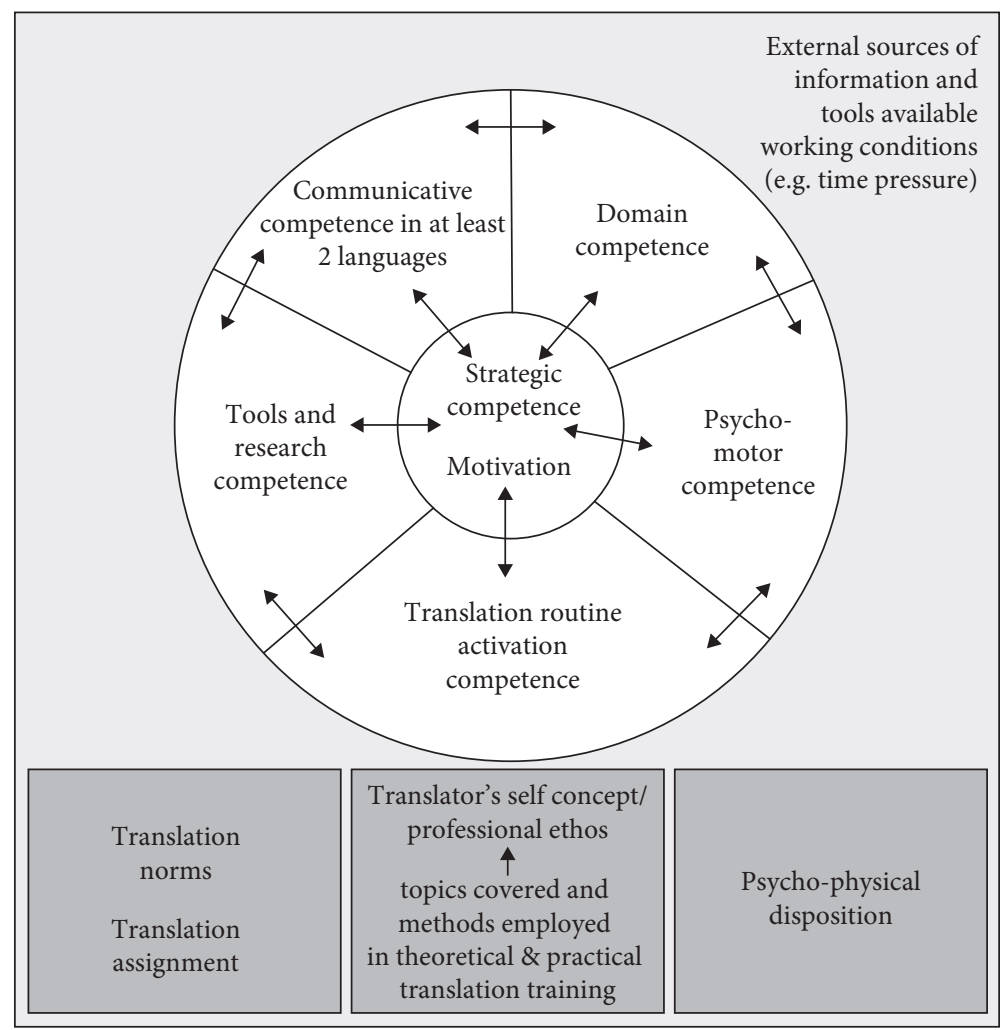

Figure 1. Translation competence model (Göpferich 2008, 155, reprinted with the permission of Narr Verlag) 
translation routine activation competence, tools and research competence and, above all, strategic competence. They were assumed to be the specific sub-competencies that distinguish highly competent translators from persons without any translation-specific training. In TransComp, they were therefore selected as dependent variables.

Tools and research competence comprises the ability to use translation-specific conventional and electronic tools. Translation routine activation competence comprises the knowledge and the abilities to recall and apply certain - mostly language-pair-specific - (standard) transfer operations (or shifts). Strategic competence controls the employment of the sub-competencies mentioned above. As a meta-cognitive competence, it sets priorities and defines hierarchies between the individual sub-competencies, leads to the development of a macro-strategy in the sense of Hönig (1995), and ideally subjects all decisions to this macro-strategy. How strictly translators adhere to employing this macro-strategy is assumed to depend on their strategic competence and their situation-specific motivation.

Against the background of DST, the following assumptions can be made with regard to the development of these three variables in relation to each other. The successful application of strategic competence requires a large amount of cognitive resources in working memory because it involves taking into account a larger context with many potential factors that may become relevant for successful decision making. As long as a critical mass of recurrent aspects of the translation process has not become automatized, such as typical language-pair specific transpositions, the execution of the respective tasks takes up so much working memory capacity that innovative (creative) strategic behaviour is not possible, or possible only to a limited extent. If creative behaviour nevertheless occurs, its results can be expected to be suboptimal because of the lack of cognitive resources for their critical evaluation.

In TransComp, different measures were used both for strategic (or creative) behaviour and for translation routine behaviour. One measure of strategic behaviour was the extent to which participants in the study proceeded in a manner which showed that they were aware of, or (systematically) developed an awareness of, the criteria that a specific target-text (TT) section has to fulfil in order for it to be an adequate correspondent for the respective ST unit. Proceeding in a strategic manner in this sense can thus be regarded as the opposite of guessing, a behaviour to which participants frequently resort when they are not aware of the criteria to be fulfilled by an adequate TT version. The degree to which participants proceed in a strategic manner was measured by analyzing the individual steps and reflec- 
tions made by the participants in problem-solving processes ${ }^{2}$ and determining whether reflections dominated in them which could be categorized as criteriaguided and clearly goal-oriented or not. If criteria-guided steps dominated and the result of the problem-solving process was correct, the process was classified as strategic. This was the case, for example, if the translator, while searching for a term in the target language, was able to enumerate the criteria by which an adequate rendering could be identified. If non-criteria-guided steps dominated, such as guessing, taking the first equivalent given in a dictionary, etc., and the result was not acceptable, the process was classified as non-strategic. All other cases were classified as indeterminable. ${ }^{3}$

A second measure of strategic competence was the participants' ability to switch between a routine mode of translation, assumed to involve low cognitive effort, and a creative and cognitively more demanding mode of translation. From a DST perspective, these two modes of translation can be interpreted as follows. Novices have difficulty being creative because the cognitive resources they have available are needed, to a large extent, for routine tasks (e.g., finding matches for words they are not familiar with or constructions which have no formal equivalent in the target language). Experts, however, have automatized many routine tasks and even tasks which, for a novice, would be regarded as creative, because they have already been exposed to similar problems in their professional lives and have thus developed usable problem-solving schemata. As a consequence, they can be expected to show high routine values (measured, for example, in terms of fluency) as well as high creativity values (measured in TransComp in terms of criteria such as creative shifts, non-fixedness, and rareness of the solutions found) because their routine releases a great deal of working memory capacity which can then be invested into being creative. The relationship between routine and creativity involved in the solution of translation problems can be visualized in what Bayer-Hohenwarter $(2012,187)$ terms "creativity/routine profiles". They are based on fine-grained translation product and process analyses, which are described in detail in Bayer-Hohenwarter (2012; for a summary, see Göpferich 2011).

The third measure of strategic behaviour in contrast to routine behaviour was the distribution of cognitively demanding decisions in relation to cognitively less demanding or routine decisions, as investigated by Prassl (2010). Following the decision-making typology by the psychologists Jungermann, Pfister, and Fischer

2. A detailed description of how problem-solving processes were identified is provided in Göpferich (2010).

3. For a more detailed description of the procedure of analysis and examples of problem-solving processes and their classification, see Göpferich (2010 and 2011). 
(2005), Prassl classifies decision-making processes, according to the cognitive effort involved in them, into routinized decisions, stereotype decisions, reflected decisions and constructed decisions. Routinized and stereotype decisions can be categorized as decisions involving low cognitive effort, and reflected and constructed decisions as decisions involving high cognitive effort (Prassl 2010).

A very obvious measure of translation routine was the number of comprehension problems the participants encountered in their translation processes. The lower the number of problems, the more routine was involved in ST comprehension (see Göpferich 2010 for further details).

\section{The results interpreted in the light of DST}

Our first measure of strategic behaviour, the degree to which the participants proceeded in a goal-oriented manner as opposed to mere guessing, did not reveal any developmental progress from the students' first to their fourth semesters. In their problem-solving processes, non-strategic steps dominated. In the professional translators' behaviour, however, a dominance of strategic behaviour could be observed (see Göpferich 2011; cf. also Angelone 2010).

What could be observed in TransComp over the time span from the students' first semester (novices) to their fourth semester, however, was an increase in their L2 competence, which is reflected in a decrease of comprehension problems, as shown in Figure 2.

Figure 2 also shows that, contrary to expectations, the total number of translation problems as well as the number of production problems and combined (comprehension and production) problems does not decrease from the first to the fourth semester, whereas the numbers in all categories are significantly lower for the professional translators than for the two student groups. From a DST perspective, this stagnation in L1 production competence development could be an indicator that target-language writing skills have not yet reached a level that releases enough working memory capacity for the application of more complex strategic measures or simply that working memory capacity is still allocated to other subcompetencies, such as receptive ones, to the extent that target-language production cannot be addressed in a strategic manner. 


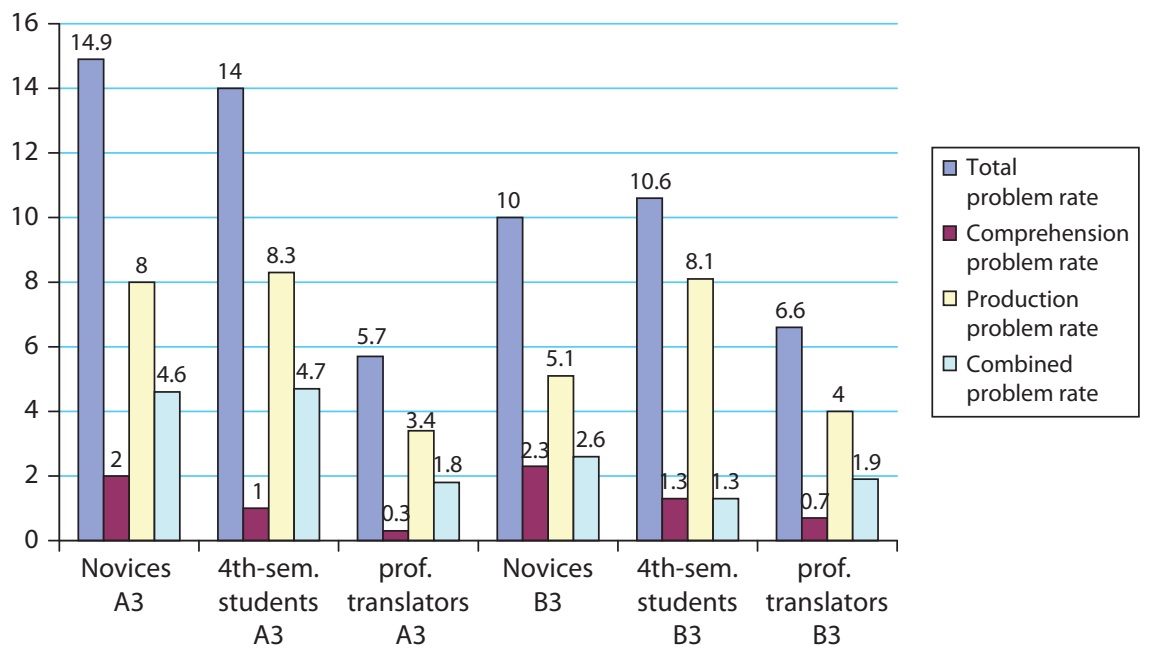

Figure 2. Number of different problem types encountered in the translation process (per 100 ST words $)^{4}$

Against this background, let us turn to the development of the participants' creativity/routine profiles. As Bayer-Hohenwarter (2012, 211-212) found, professional translators can combine high creativity scores with high routine scores, as had been expected. This is an indicator of their "switch competence" (see also the summary in Göpferich 2011). Students, in contrast, tend to be either creative with a low degree of routine or to show routine behaviour with a low degree of creativity. The creativity/routine profiles in Bayer-Hohenwarter $(2012,211)$ also show that the students' average creativity and routine values hardly change from their first to their fourth semesters. Only in the fifth semester can an increase in creativity be observed, which is, however, combined with a decrease in routine.

What we must take into account in future analyses, however, is that there is interpersonal variability between students, as the creativity/routine profiles for two individual students, KNI and CHA, show in Figure 3 (Bayer-Hohenwarter 2011).

In neither of the two students' profiles can a development towards the professional translators' creativity/routine profiles be seen (there are, however, individual students which show a development; Bayer-Hohenwarter 2011). The two students' behaviours, however, differ considerably. KNI shows high creativity values combined with low routine values; in CHA's case, the opposite holds true. If the combination of high routine with high creativity is an attractor state in the dynamic system of translation competence, TransComp at least reveals that the

4. $\mathrm{A} 3$ and $\mathrm{B} 3$ are two operating-instructions texts. 

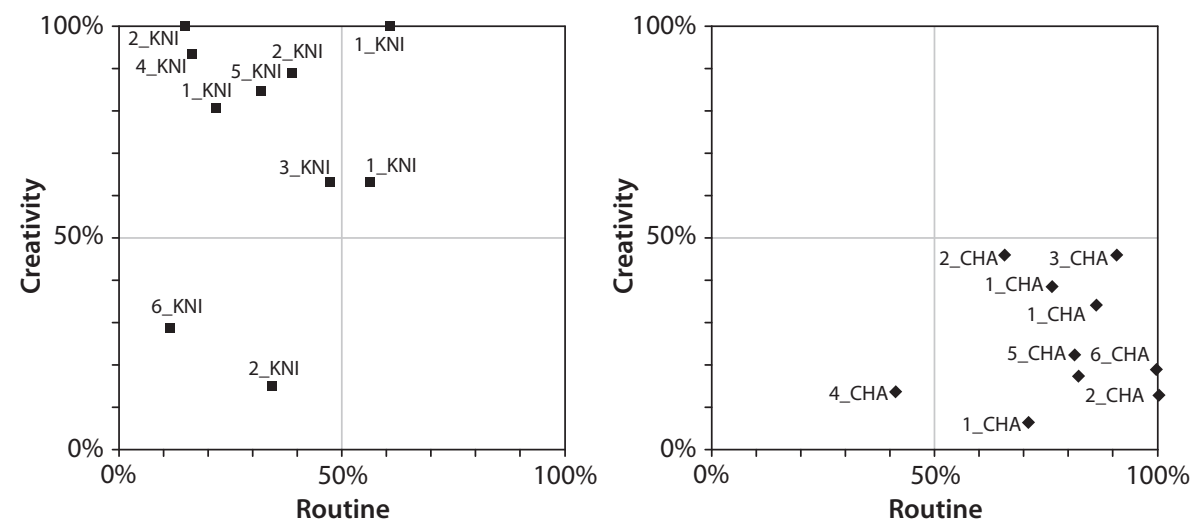

Figure 3. Individual creativity/routine profiles for two individual students: KNI and CHA (Bayer-Hohenwarter 2011)

student participants of the study had not yet achieved this state after the three years of their BA program.

Let us now turn to our third measure of strategic competence, the cognitive effort involved in decision-making processes and to what extent these were successful.

Table 1 shows how many of the decisions the student participants made in each semester (1st-5th semester) fall into either the category "low-effort decisions" or "high-effort decisions" (both in absolute numbers and in percent). It also shows how many of the decisions in each category were successful (values in darker columns). The last column gives the total of successful decisions.

Table 1 shows that the professional translators invest less effort in decision making than the students $\left(\chi^{2}=17.61, p \leq 0.001\right)$. Whereas only one fifth of the students' decisions fall in the category of low-effort decisions, more than a third of the professional translators' decisions do. Here it is noteworthy to mention that the professional translators' low-effort decisions are considerably more successful than the students. More than half of the professional translators' decisions in this category are successful whereas the students' success rate is only about one third. For high-effort decisions, however, the professional translators' success rate is only slightly higher than the students. Here, it was expected that the professional translators' success rates would increase with the cognitive effort invested (cf. the preliminary findings of Prassl 2010). That this is not the case suggests that the professional translators' evaluation competence lags behind what was expected of them or that they do not yet have enough cognitive resources left for satisfactory evaluation. They seem to lack the ability to apply relevant criteria in their evaluation processes. This is something that an expert in the sense of expertise research should be able to do. That they are not able to do so suggests that they have not 
Table 1. Frequency and success of decisions involving relatively low vs. relatively high cognitive effort (Prassl in progress)

\begin{tabular}{|c|c|c|c|c|c|}
\hline & \multicolumn{2}{|c|}{$\begin{array}{l}\text { Decisions involving low cognitive effort } \\
\text { (routinized and stereotype) }\end{array}$} & \multicolumn{2}{|c|}{$\begin{array}{l}\text { Decisions involving high cognitive effort } \\
\text { (reflected and constructed) }\end{array}$} & \multirow{2}{*}{$\begin{array}{l}\text { total of correct decisions } \\
\text { number (absolute) and } \\
\text { percentage }\end{array}$} \\
\hline & $\begin{array}{l}\text { number (absolute) } \\
\text { and percentage }\end{array}$ & $\begin{array}{l}\text { correct decisions } \\
\text { number (absolute) } \\
\text { and percentage }\end{array}$ & $\begin{array}{l}\text { number (absolute) } \\
\text { and percentage }\end{array}$ & $\begin{array}{l}\text { correct decisions } \\
\text { number (absolute) } \\
\text { and percentage }\end{array}$ & \\
\hline students & 43 & 10 & 154 & 50 & 60 \\
\hline 1st semester & $21.8 \%$ & $23.3 \%$ & $78.2 \%$ & $32.5 \%$ & $30.5 \%$ \\
\hline students & 33 & 15 & 147 & 44 & 59 \\
\hline 2nd semester & $18.3 \%$ & $45.5 \%$ & $81.7 \%$ & $29.9 \%$ & $32.8 \%$ \\
\hline students & 16 & 4 & 58 & 17 & 21 \\
\hline 3rd semester & $21.6 \%$ & $25 \%$ & $78.4 \%$ & $29.3 \%$ & $28.4 \%$ \\
\hline students & 18 & 8 & 49 & 15 & 23 \\
\hline 4th semester & $26.9 \%$ & $44.4 \%$ & $73.1 \%$ & $30.6 \%$ & $34.3 \%$ \\
\hline students & 8 & 5 & 45 & 11 & 16 \\
\hline 5 th semester & $15.1 \%$ & $62.5 \%$ & $84.9 \%$ & $24.4 \%$ & $30.2 \%$ \\
\hline students & $118^{*}$ & $42^{* *}$ & $453^{*}$ & $137^{* *}$ & 179 \\
\hline 1st-5th semester & $20.7 \%$ & $35.6 \%$ & $79.3 \%$ & $30.2 \%$ & $31.3 \%$ \\
\hline professional & $95^{*}$ & $53^{* *}$ & $185^{*}$ & $60^{* *}$ & 113 \\
\hline translators & $33.9 \%$ & $55.8 \%$ & $66.1 \%$ & $32.4 \%$ & $40.4 \%$ \\
\hline
\end{tabular}

${ }^{*} \chi^{2}=17.61, p \leq 0.001{ }^{* *} \chi^{2}=17.34, p \leq 0.001$ 
yet achieved expert status. Table 1 also shows that the professional translators' decision-making processes are almost $10 \%$ more successful than the students' processes on average. In all decision-making categories (apart from constructed decisions, which occur only very rarely), the students are considerably less successful than the professionals. The discrepancy in the success rates between the two groups of participants is highest for decisions involving low cognitive effort. In this category, the discrepancy is more than $20 \%$, whereas in the category of decision-making types involving high cognitive effort, it is only about $2 \%$. The fact that professional translators decide more frequently without investing much cognitive effort and are nevertheless more successful also points to their higher routine, as was the case with Bayer-Hohenwarter's creativity/routine profiles.

\section{Discussion and conclusion}

While the professional translators clearly outperformed the student group with regard to all variables analyzed, they were found to have not yet achieved expertise, the highest level of competence. The student participants' competence development over the first two-thirds of their bachelor's program did not follow a linear path with regard to the translation-specific variables; there seemed to be stagnation in the development of their translation competence. Potential explanations for these observations are provided in Göpferich (2011). In the following, let us embed them in the framework of DST.

Let us first consider the professional translators' competence which lagged behind our expectations (cf. their unexpectedly low percentages of correct decisions in Table 1). The professional translators' stagnation in competence development, albeit at a high level, has many possible causes. It may be due to a lack of deliberate practice (Shreve 2006, 29) in their usual work assignments, which were possibly dominated by routine tasks, or a lack of continuous feedback on their translations, which would be necessary for their competence to develop continuously (Shreve 1997, 128; 2006, 29, 32). Furthermore, this stagnation may have resulted from the fact that they applied criteria (e.g., equivalence-oriented ones) that deviated from our functional approach, ${ }^{5}$ or perhaps it was simply a lack of motivation in the experiment situation that led to these surprising results. No matter the cause, they seem to have achieved an attractor state that is so strong that a considerable amount of specific training would be needed to help them overcome this state. This training could take the form of intensive work sessions in which they can discuss their products and processes with "masters", who can point out

5. The translation assignments required a functional approach. 
their weaknesses to them and show them how these problems can be overcome in a kind of cognitive apprenticeship (Kellogg 2008).

The students definitely have not yet reached the attractor state in which routine and creativity are in an equilibrium state. They show either a high degree of routine or a high degree of creativity. Furthermore, their evaluation competence in both areas does not seem to have developed to a satisfactory degree, which can be seen from the relatively large number of decisions which led to unacceptable solutions. This observation can be explained within the DST paradigm combined with an effort model. The effort model provides a framework which sets a limit to the values that the different variables can take in the dynamic system of translation competence. In order to be able to integrate highly demanding cognitive processes into their problem solving, translators must have proceduralized a certain amount of recurrent translation sub-tasks, which then releases cognitive capacity for more demanding tasks. This proceduralization requires translators to deliberately practice their coping skills with challenging tasks, on which feedback must also be provided. Occasions for this deliberate practice with feedback were not provided in the first four semesters of the bachelor's program at the Department of Translation Studies of the University of Graz, in which the student participants were enrolled. Students of this program do not start translating before their 5th semester (in the course "Translatorische Basiskompetenz I") as the first four semesters are mainly devoted to the development of language competence as well as lectures on linguistic and translation theory. In such lectures, they acquire only declarative knowledge, which does not lead to cognitive relief in the translation process if there is no occasion to proceduralize the declarative knowledge by practice.

Another explanation for the seeming stagnation in the students' competence development, which is connected with the first explanation, may be that the students' problem awareness has grown during their first semesters (e.g., due to their exposure to translation theories in their lectures), whereas their problem-solving competence lags behind, again due to a lack of practice. Increasing problem awareness in combination with a lack of routine requires a lot of working memory capacity for problem solving that leaves no room for more complex cognitive processes. As a consequence, only very short translation units can be tackled and only few relevant criteria can be taken into account, which is detrimental to translation quality (see Göpferich 2008, 168-178 for a summary of findings on these phenomena). With regard to the students' translation competence, especially their strategic competence, the following hypothesis can be derived from this in the light of DST. Their translation competence development as a whole has not stagnated over the first semesters; rather, there has been a shift in the allocation of their cognitive resources that does not yet have an effect on the translation quality of their output and could therefore not be measured using the instruments we applied. This may 
be due to the fact that the shift in the allocation of cognitive resources has not been accompanied by a sufficient level of proceduralization of more complex recurrent tasks that would allow more complex solution schemata to be taken into account without using up working memory storage capacity. This phenomenon is accounted for by Ericsson and Kintsch's (1995) concept of "long-term working memory". Accessing knowledge from there is less laborious than having to maintain it in an active state in short-term working memory, freeing short-term working memory capacity for other tasks (Kellogg 2008, 15).

Against this background, it seems worthwhile to investigate whether the quality of the students' translation products increases once their increased problem awareness is accompanied by a higher amount of routine acquired in practical translation courses. Although Figure 2 does not show an increase in problem awareness from the first to the fourth semester, we have to take into account a phenomenon that Jääskeläinen $(2002,111)$ calls the "developmental hypothesis". According to this hypothesis, problems that become the object of conscious decision-making processes in translation do not decrease in number as a translator's competence increases but change in quality in the course of time (see also Dreyfus and Dreyfus 1986).

Furthermore, it must be taken into account that translation competence acquisition is combined with language acquisition (Bergen 2009). The findings with regard to comprehension problems, for example, suggest that stagnation in the development of translation competence may be accompanied by a development (or perhaps even an accelerated development) of language competence. This underlines once more that the acquisition of competences always has to be seen against the background of other competences whose development may accompany the process.

The discussion of the results obtained in the TransComp study shows that they can be interpreted in the DST framework and are compatible with it. However, the variables or sub-competencies whose development was analyzed are still too few to give us an insight into the specific sub-competencies that get prioritized when another sub-competence of translation becomes automatized, as well as insight into whether these are always the same sub-competencies for each individual. We also only have a rough idea of which sub-systems are precursors of other subsystems and which ones are connected growers. However, it would be highly interesting to find out more about this. If we did, it would then give us an indication of what skills and sub-skills need to be practised in parallel or in what sequential order. With regard to translation, the results from the TransComp longitudinal study, for example, suggest that the more complex strategic sub-competence does not develop until less complex sub-competencies, such as receptive competence in the L2, and potentially also production competence in the L1, have reached a certain threshold value. To learn more about these interconnections, we not only need to refine our measuring instruments, we also need to gain deeper insights 
into the competencies to which translation competence in the narrower sense is related. Disciplines with which cooperation seems to be most fruitful here are bilingualism research, foreign language teaching and developmental psychology. Bilingualism research and foreign language teaching could give us insight into the development of linguistic sub-competencies that, though not translation-specific, may have an impact on the development of translation competence. In developmental psychology, non-linear development of motor and cognitive competencies has been observed and successfully interpreted in the DST paradigm (Thelen and Smith 1994). As I hope to have shown in this chapter, adopting this paradigm in translation studies may be a promising endeavour.

\section{References}

Angelone, Eric. 2010. "Uncertainty, Uncertainty Management and Metacognitive Problem Solving in the Translation Task." In Translation and Cognition, ed. by Gregory Shreve, and Eric Angelone, 17-40. Amsterdam: John Benjamins. DOI: 10.1075/ata.xv.03ang

Bayer-Hohenwarter, Gerrit. 2011. "The Development of Creativity Analysed: A Plea for More

Fine-grained Analyses in Translation Process Research." Paper at the 2nd International

Research Workshop "Methodology in Translation Process Research." University of Giessen, 27-29 July, 2011.

Bayer-Hohenwarter, Gerrit. 2012. Translatorische Kreativität: Definition, Messung, Entwicklung

[Translationswissenschaft, 8]. Tübingen: Narr.

Bergen, David. 2009. "The Role of Meta-Cognition and Cognitive Conflict in the Development of Translation Competence." Across Languages and Cultures 10 (2): 231-250.

DOI: 10.1556/Acr.10.2009.2.4

de Bot, Kees, Wander Lowrie, and Marjolijn Verspoor. 2007. "A Dynamic Systems Theory Approach to Second Language Acquisition." Bilingualism: Language and Cognition 10 (1): 7-21. DOI: $10.1017 /$ S1366728906002732

Dreyfus, Hubert L., and Stuart E. Dreyfus. 1986. Mind Over Machine. Oxford: Blackwell. Englund Dimitrova, Birgitta. 2005. Expertise and Explicitation in the Translation Process [Benjamins Translation Library, 64]. Amsterdam: John Benjamins. DOI: 10.1075/btl.64

Ericsson, K. Anders, and Walter Kintsch. 1995. "Long-term Working Memory." Psychological Review 102: 21-245. DOI: 10.1037/0033-295X.102.2.211

Van Gelder, Tim. 1989. “The Dynamical Hypothesis in Cognitive Science." Behavioral and Brain Sciences 21: 615-656.

Gile, Daniel. 1995. Basic Concepts and Models for Interpreter and Translator Training. Amsterdam: John Benjamins. DOI: 10.1075/btl.8

Gile, Daniel. 1997. "Conference Interpreting as a Cognitive Management Problem." In Cognitive Processes in Translation and Interpreting, ed. by Joseph H. Danks, Stephen B. Fountain, Michael K. McBeath, and Gregory M. Shreve, 196-214. Thousand Oaks, CA: Sage.

Göpferich, Susanne. 2008. Translationsprozessforschung: Stand - Methoden - Perspektiven

[Translationswissenschaft, 4]. Tübingen: Narr. 
Göpferich, Susanne. 2009. “Towards a Model of Translation Competence and its Acquisition: The Longitudinal Study 'TransComp."' In Behind the Mind: Methods. Models and Results in Translation Process Research [Copenhagen Studies in Language, 37], ed. by Susanne Göpferich, Arnt L. Jakobsen, and Inger M. Mees, 11-37. Copenhagen: Samfundslitteratur. Göpferich, Susanne. 2010. "The Translation of Instructive Texts from a Cognitive Perspective." In New Approaches in Translation Process Research [Copenhagen Studies in Language, 39], ed. by Susanne Göpferich, Fabio Alves, and Inger M. Mees, 5-55. Copenhagen: Samfundslitteratur.

Göpferich, Susanne. 2011. "From Multidisciplinarity to Transdisciplinarity: The Investigation of Competence Development as a Case in Point." MikaEL Electronic Proceedings of the KäTu Symposium on Translation and Interpreting Studies 5: 1-24. http://www.sktl.fi/@Bin/85366/ G\%F6pferich_MikaEL2001.pdf (last accessed 25/01/2015).

Göpferich, Susanne, Gerrit Bayer-Hohenwarter, and Hubert Stigler (eds). 2011. TransComp The Development of Translation Competence. Corpus and Asset-Management-System for the Longitudinal Study TransComp. Graz: Karl-Franzens-Universität. http://gams.uni-graz.at/ container:tc (last accessed 25/01/2015).

Hönig, Hans G. 1995. Konstruktives Übersetzen [Studien zur Translation, 1]. Tübingen: Stauffenburg.

Jääskeläinen, Riitta 2002. “Think-Aloud Protocol Studies into Translation: An Annotated Bibliography." Target 14 (1): 107-136. DOI: 10.1075/target.14.1.05jaa

Jungermann, Heinrich, Hans Rüdiger Pfister, and Katrin Fischer. 2005. Die Psychologie der Entscheidung. Eine Einführung, 2nd ed. Munich: Elsevier.

Kellogg, Ronald T. 2008. "Training Writing Skills: A Cognitive Developmental Perspective.” Journal of Writing Research 1 (1): 1-26.

Prassl, Friedrike. 2010. “Translators' Decision-Making Processes in Research and Knowledge Integration." In New Approaches in Translation Process Research [Copenhagen Studies in Language, 37], ed. by Göpferich, Susanne, Fabio Alves, and Inger M. Mees, 57-81. Copenhagen: Samfundslitteratur.

Prassl, Friederike. In progress. Translatorische Recherche und Wissensintegration. (Dissertation). Graz: Karl-Franzens-Universität Graz/ITAT.

Schäffner, Christina, and Beverly Adab. 2000. "Developing Translation Competence: Introduction." In Developing Translation Competence, ed. by Christina Schäffner, and Beverly Adab, vii-xvi. Amsterdam: John Benjamins. DOI: 10.1075/btl.38.01sch

Shreve, Gregory M. 1997. "Cognition and the Evolution of Translation Competence." In Cognitive Processes in Translation and Interpreting, ed. by Joseph H. Danks, Stephen B. Fountain, Michael K. McBeath, and Gregory M. Shreve, 20-136. Thousand Oaks, CA: Sage. Shreve, Gregory M. 2006. "The Deliberate Practice: Translation and Expertise." Journal of Translation Studies 9 (1): 27-42.

Thelen, Ester, and Linda B. Smith. 1994. A Dynamic Systems Approach to the Development of Cognition and Action. Cambridge, MA: MIT Press.

Verspoor, Marjolijn, Kees de Bot, and Wander Lowie. 2004. "Dynamic Systems Theory and Variation: A Case Study in L2 Writing." In Words in their Places, ed. by Hendrik Aertsen, 407-421. Amsterdam: VU.

Verspoor, Marjolijn, Wander Lowrie, and Marijn van Dijk. 2008. "Variability in Second Language Development from a Dynamic Systems Perspective." The Modern Language Journal 92 (2): 214-231. DOI: 10.1111/j.1540-4781.2008.00715.x 


\title{
Applying a newswriting research approach to translation
}

\author{
Maureen Ehrensberger-Dow and Daniel Perrin \\ Zurich University of Applied Sciences, Switzerland
}

\begin{abstract}
Translation is a situated activity that involves more than simply producing target texts from source texts. In order to understand what translators actually do when they translate, their psycho-biographies as well as the social setting of the workplace and the contextual resources must be considered. In this chapter, we outline how a mixed-method approach originally developed to study the newswriting processes of journalists at their workplaces can be applied in translation process research. We argue that progression analysis, which combines keystroke logging, screen recordings, eye-tracking, and cue-based retrospective verbalization, can be profitably used along with version analysis to gain insights into cognitive aspects of the translation process.
\end{abstract}

Keywords: translation process, newswriting research, progression analysis, eyetracking, version analysis

\section{Introduction}

From an almost exclusive focus on products, translation studies has moved towards examining processes (including cognitive aspects) and the effects of those processes on the quality of products (cf. Lee-Jahnke 2005). The latter are the result of the interaction between societal expectations of what translations should be and translators' emergent practices and translation competence that allow them to produce acceptable translations in a given setting or situation within temporal and economic constraints (cf. Archer 2003).

In this chapter, we show how research with another group of language professionals, namely journalists, can be applied to investigate the situated activity of translation. Since we are interested in releasing expert knowledge by gaining an understanding of what language professionals actually do, we have chosen a rather complex method mix based on an approach called progression analysis. 
A case study and comparison data from the corpus of a longitudinal project ${ }^{1}$ are presented to illustrate the applicability of this approach to translation.

\section{The relevance of newswriting research for translation studies}

Writing research conceptualizes writing as the production of texts, as cognitive problem solving, and as the collaborative practice of social interaction and meaning making (Juzwik et al. 2006; Rijlaarsdam et al. 1996). Newswriting research sees text production as a reproductive process in which professional journalists transform source texts into target texts (Jacobs 2011; Perrin 2012; Van Hout, Pander Maat, and De Preter 2011). This happens at collaborative workplaces, in processes of goal setting, planning, formulating, and revising. Reading phases are integrated in the process: journalists read source texts and their emerging own text before, while, and after writing (Perrin 2013). The parallels to the activities of translators during the translation process would seem obvious.

The present state of writing research results from two paradigm shifts, which have their correlates in translation studies. In a first paradigm shift, the focus of interest moved from the product to the process. A second paradigm shift took research from the lab to the field, as researchers moved from testing subjects with experimental tasks to ethnographic approaches of investigating communities of practice. Later, ethnography was complemented by recordings of writing activities, such as keystroke logging. The research reviewed below reflects the influence of these paradigm shifts on methods used in empirical writing research and translation process research.

\subsection{Parallels between writing and translation process research methods}

Not only are most news texts and translations now produced on computers, many are transmitted to their audiences solely in electronic form. Recent writing and translation process research has been quick to exploit the possibilities offered by the technological developments that have dramatically altered text production and translation processes. At each phase of their production, texts and translations can be reconstructed and analyzed with respect to various characteristics by using non-invasive techniques such as keystroke logging (e.g., Van Waes and Leijten 2006 in writing research and Jakobsen 2006 in translation research). Logfiles are useful for investigating pauses and micro-changes at different stages of the writing or translation process,

1. See www.linguistik.zhaw.ch/iued/capturing for further information about the Capturing Translation Processes project. 
although they provide no information about what a writer or translator might be doing when they are not entering text into the computer or using the mouse. However, other methods can overcome this limitation to provide richer information about the process. For example, screen capture software records all the changes that take place on the computer screen during a writing or translation process. Eye tracking, which monitors eye movements and gaze intensity, can provide information about the focus of attention, such as when a writer or translator switches screens to refer to a source of information or to check for a word in an online thesaurus.

From a product perspective, version analysis is the method of collecting and analyzing data in order to reconstruct the changes that a text undergoes during its production or to compare linguistic differences in texts produced by various individuals. The basis for comparing versions is text analysis. Version analysis traces linguistic products and elaborates on the changes in text features from version to version throughout intertextual chains (cf. Perrin and Ehrensberger-Dow 2008, 286-287 for writing and Englund Dimitrova 2005, 143-144 for translation processes). However, version analysis alone fails to provide any information about whether the journalists or translators were conscious of their actions during text production and revision. To generate such knowledge, additional methods such as self-report or interviews are required.

Concurrent and retrospective verbalizations are forms of self-report that have been exploited in both writing and translation research (cf. Levy et al. 1996 and Jääskeläinen 2011, respectively). Although concurrent reports are widely used in psychology and language studies, they have been criticized for affecting the process that is being commented upon. Cue-based retrospection, performed immediately after task execution, has been identified as an ecologically valid alternative to concurrent verbalization (cf. Hansen 2006; Perrin 2003; 2006). An important advantage of this technique is that different modes of expression (writing and talking) do not have to be used simultaneously; the talking has no impact on the writing or translation process because it happens afterwards.

\subsection{Methodological considerations}

Within and across disciplines, the question as to which research method suits which research question is dealt with by methodology, the scientific cross-sectional discipline that deals with the relationship between the object of study, research question, methods, and the expected results in scientific research. Some of the methodological dichotomies include: quantitative vs. qualitative; statistical representation vs. case studies; laboratory research vs. field studies; structured questionnaires vs. observation; and single vs. multi-method approach. Whereas methodologically rigorous positions tend to concentrate on a single theory and 
method, pragmatic positions prefer to combine them in order to gain multi-perspective insights (Tashakkori and Teddlie 2003). Such a combination of methods is exemplified by progression analysis.

\section{Progression analysis in newswriting research}

Progression analysis was originally developed to investigate newswriting (Perrin 2003; 2006). It is a multi-method approach of collecting and analyzing data in natural workplace settings at different levels, in order to reconstruct text production processes as a cognitively controlled and socially anchored activity. Progression analysis operates on three levels: situation, performance, and conceptualization.

1. On a situation level, progression analysis determines through interviews and observations what the writing situation is and what experience journalists draw on to guide their actions. Important factors include the writing task, professional socialization, and economic, institutional, and technological influences on the work situation. Data on the self-perception of the journalists under investigation are obtained in semi-standardized interviews about their psychobiography, primarily in terms of their writing and professional experience, and their workplace. In addition, participatory and video observations are made of their situated activity at the workplace, including various kinds of collaboration.

2. On a level of practice or performance, directly observable actions such as revisions in a growing text are logged and analyzed. During newswriting, keystroke logging and screen recording programs, which run unobtrusively in the background behind the text editors that the journalists usually use, record every keystroke and writing movement in the emerging text. The recordings can follow the writing process over several workstations and do not influence the performance of the editing system or the journalist.

3. On the level of socio-cognitive conceptualization or reconstruction, progression analysis draws on verbal data to infer the mental structures that might have guided the writing activities observed on the second level. After writing is completed, journalists view a playback of their process and watch how their texts came into being. While doing so, they continuously comment on what they did while writing and why they did it. An audio recording is made of these cue-based retrospective verbal protocols (RVPs). It is assumed that this third level of progression analysis reveals considerations that a journalist could have made in principle, although not the sum of all and only the considerations that the journalist actually made. The cue-based RVP is transcribed 
and then encoded as the journalist's verbalization of aspects of his or her language awareness, writing strategies, and conscious writing practices.

The data from these three levels complement each other to provide a multi-perspective, vivid picture of newswriting. Visualizations of the data help with the detection of problematic points in the emerging texts, and the computer logs provide detailed information about what happens during the process at those points. Finally, the cue-based RVPs provide information about the journalists' awareness of what they are doing and why.

In sum, progression analysis allows researchers to consider all the revisions to the text as well as all of the electronic resources accessed during the production process; to trace the development of the emerging media item; and finally to reconstruct collaboration at media workplaces from different perspectives. The focus of progression analysis is the socially embedded cognitive and manifest processes of writing by individuals: their situated activity in context. The next section explains how progression analysis can be and has been applied in the context of translation, in order to obtain a deeper understanding of the decisions that translators make during the translation process.

\section{Tracing translation processes with progression analysis}

In a longitudinal study of translation processes ${ }^{2}$, we have applied progression analysis at all three levels: the situation surrounding the translation activity (4.1); the translation movements and practices that the translators engage in (4.2); and the translation strategies that can be inferred from the translators' comments about their translation processes (4.3). In addition, we have used version analysis to investigate the emergence of individual target texts and to compare translations of the same source text produced by different translators under controlled conditions (4.4). The detailed analysis of the case study presented below exemplifies how progression analysis can be used in translation process research.

4.1 First level of progression analysis: the translation situation

The translation situation includes the linguistic, educational, and professional background information of the translators as well as the setting and the task demands. The woman (ET0413) whose process is presented below was one of five

2. Financial support from the Swiss National Science Foundation for this project is gratefully acknowledged (Grant 13DFD3_124653/1). 
paid volunteers who had recently completed a four-year undergraduate translation degree program and was enrolled in the first semester of a post-graduate interpreting program. Her first language (L1) was German, her strongest foreign languages (L2) were English and Italian, and her less active language was French. She had also taken part in data collection for the research project in her undergraduate program, so was accustomed to having her translation processes monitored. She was told that her translation process was being recorded in order to test the new eye-tracking equipment. ${ }^{3}$

The setting for the translation process was the institute's usability lab; the computer that the participant was working at had the same MS Office environment that she was familiar with from other institute computer workstations as well as access to the internet, institute online dictionaries, and other university library resources. After a brief session in the usability lab to become familiar with the setting and the eye-tracking equipment (Tobii T60), she took part in three recording sessions spread over a period of two months: the process considered here was done in the first session.

The participant was given as much time as she needed to translate a German journalistic source text of about 115 words into English (see Appendix A). The translation brief was provided, as was the link to the article in the online publication that it originally appeared in. After about 20 minutes, she indicated that she was finished. Immediately afterwards, the screen recording of her translation process was retrieved on a laptop computer in a room adjacent to the usability lab, and she commented on what she had done while she viewed the recording of her process replayed in real time. A research assistant was present during the verbalization but sat where the computer screen could not be seen easily, not only to increase the ecological validity of using verbalization as a method but also to prompt the participant to continue talking whenever necessary. The participant was asked to talk about what she was watching but was not asked to provide explanations of her actions or given any indication of what type of comments might be of particular interest to the researchers.

\subsection{Second level of progression analysis: translation activities and practices}

Keystroke logging and screen capture software record the development of the emerging translation, all of the revisions to it as well as the search terms and electronic resources that are accessed during the translation process. In the process considered here, the translation of the 5th sentence of the source text (ST) is examined in detail:

3. See Ehrensberger-Dow and Künzli (2010) for further details. 
Laut dem EU-Schnellwarnsystem RAPEX erwiesen sich 2006 erstmals Spielzeuge als gefährlicher als Elektrogeräte. ${ }^{4}$

In the final version of ET0413's target text (TT), the corresponding sentence appeared as:

According to the EU rapid alert system RAPEX, toys had proven more dangerous than electronic devices for the first time in 2006.

It took the translator just over two minutes to produce the first complete version of the sentence, measured from when she typed the capital letter at the start of the sentence until she typed the full stop at the end. During that time, she interrupted the flow of her translation twice to do research and numerous times to make revisions, such as deleting or inserting letters and/or words. Each step resulted in a new version of the sentence (see Appendix B). After she finished her first draft, as indicated by typing the final punctuation of the last sentence of the target text, she worked through her translation in a short revision phase, making four more revisions to the 5th sentence of her TT.

Although the first two revisions in the introductory phrase of the TT sentence seemed to be related to typing slips (typos), the following revisions, especially those related to two time adverbials, seem to reflect difficulties related to translation direction. The multi-method approach of progression analysis allows us to reconstruct the translation process, as presented below, with data elicited with each method allowing insights into the various steps of the overall process.

\subsubsection{Computer logging}

The keystroke logging software InputLog, developed by the writing researchers Van Waes and Leijten (2006), was used to record the timing of keystrokes, mouse movements, clicks, wheel movements, references to URLs, and pauses during the target-text production process. The software is compatible with many user interfaces and has no restrictions with respect to window size or layout. With the log data, it is possible to reconstruct the phases of the translation process and to determine the time required to research and produce each sentence of the target text (Table 1). Excluding the time spent on research, the translator took longer to complete the 5th sentence (i.e., time between writing the first lettter and the final punctuation) than any other sentence of the target text, although it was not the longest.

A challenge in carrying out newswriting or translation research in workplace settings is to ensure that the logging software records not only the timing of keystrokes, mouse movements, and shifts between windows but can also trace changes to an emerging translation that result from the use of tool functionalities such

4. See Massey and Ehrensberger (2010) for a discussion of search behavior related to this sentence. 
Table 1. Temporal information about ET0413's translation process from the logfile

\begin{tabular}{|c|c|c|c|c|c|}
\hline Phase & Target text & $\begin{array}{l}\text { Words } \\
\text { (ST) }\end{array}$ & $\begin{array}{l}\text { Overall duration } \\
\quad(\mathrm{msec})\end{array}$ & $\begin{array}{c}\text { Research } \\
(\mathrm{msec})\end{array}$ & $\begin{array}{l}\text { TT production } \\
\text { (msec) }\end{array}$ \\
\hline Orientation & & & 101203 & 31281 & 0 \\
\hline \multirow{9}{*}{ 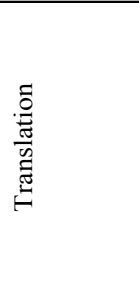 } & Title & 4 & 5875 & 0 & 5875 \\
\hline & 1st sentence & 16 & 74047 & 22204 & 51843 \\
\hline & 2nd sentence & 25 & 201672 & 91063 & 110609 \\
\hline & 3rd sentence & 10 & 115953 & 57343 & 58610 \\
\hline & 4th sentence & 15 & 153859 & 52281 & 101578 \\
\hline & 5th sentence & 13 & 133875 & 15407 & 118468 \\
\hline & 6th sentence & 14 & 70593 & 21828 & 48765 \\
\hline & 7th sentence & 16 & 114797 & 48186 & 66611 \\
\hline & URL link & 1 & 7046 & 0 & 7046 \\
\hline Revision & & & 149734 & 0 & 149734 \\
\hline
\end{tabular}

as cut, paste, auto-correct, and match (i.e., from translation memory software). In the absence of customized logging software that automatically records that information, screen recordings can be made with software such as Camtasia Studio and those types of actions can be transcribed manually. The function of mouse movements and clicks relative to the emerging text usually becomes apparent through an examination of the screen recordings. In addition, detailed information about electronic resource and tool use can be obtained from the screen recordings. Analyzing these recordings takes far more time than an automatic analysis of logfiles but adds relevant data about the process.

\subsubsection{S-notation}

Although micro changes during text production can be traced in the output of logging software, the degree of detail can make it extremely difficult to gain an overview of the process. The presentation of individual revision steps (such as those in Appendix B) may be useful for illustrative purposes but is extremely time- and space-consuming to produce. To address this problem in writing research, a system known as S-notation was developed to depict the steps in logfiles in order to simplify the detail analysis of a text production process (Kollberg 1998; Kollberg and Severinson-Eklundh 2001). Whenever a writer or translator interrupts the flow of text production to delete something in the text (e.g., with a backspace, delete, or cut command) or to insert something at another place, S-notation indicates this with a numbered break symbol in the text. Deleted text is indicated in square brackets, and insertions in curly braces. The order of any given writing interruption is provided by a subscript to the right of the break symbol $\left.\right|_{n}$ and by matching superscripts on either side of the brackets ${ }^{n}[]^{\mathrm{n}}$ or braces ${ }^{\mathrm{n}}\{\}^{\mathrm{n}}$.

Figure 1 shows the revisions in ET0413's translation process compressed with $\mathrm{S}$-notation. The almost linear increase in the numbers over the first four sentences of the target text and at the beginning of the 5th sentence indicates a relatively 


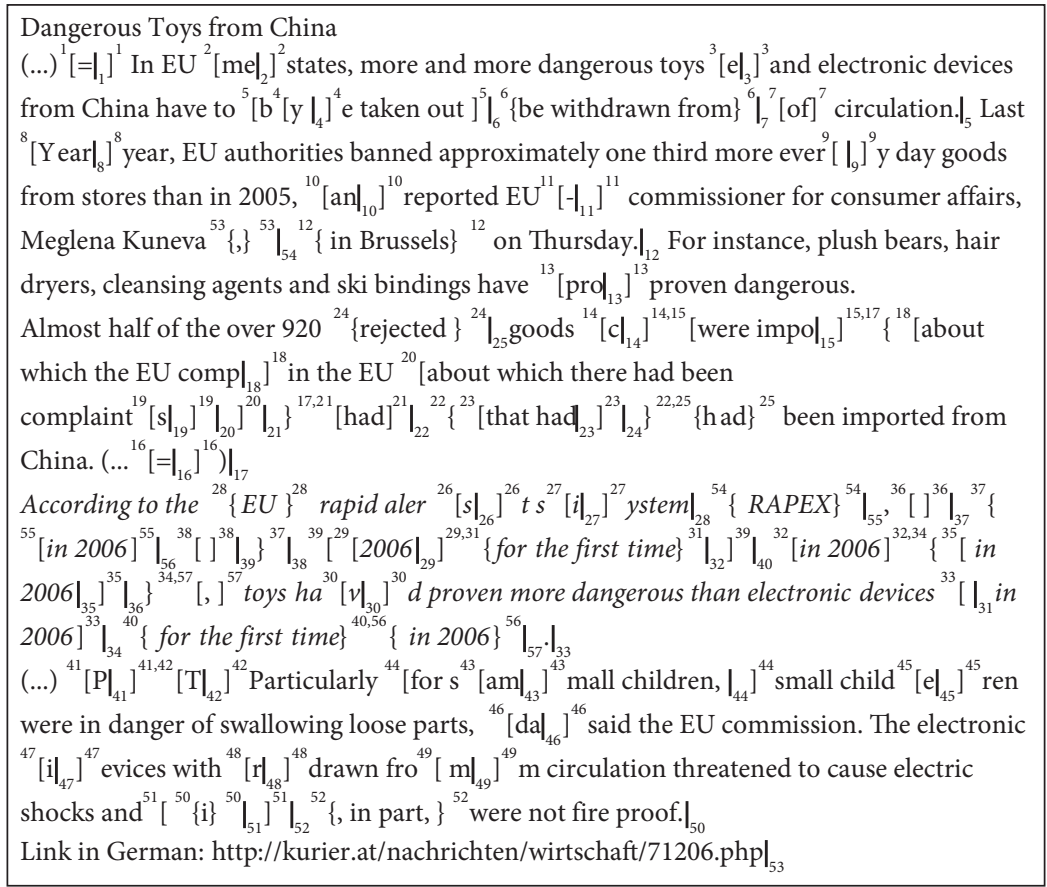

Figure 1. S-notation of ET0413's translation process (5th sentence indicated with italics)

smooth translation process. In the second part of the 5th sentence, the numbers are out of sequence, although they again increase linearly in the last two sentences of the TT. One way to visualize this progression of TT production is explained in the next section.

\subsubsection{Progression graphs}

The broader pattern of the revisions that are captured in the logfiles and compressed with S-notation can be traced in a graph that reflects the development of the translation over time. Perrin (2003) developed progression graphs such as the one in Figure 2 to provide an overview of the course of writing. The order of the revisions in the writing process is shown on the horizontal axis, and the position of the revisions in the final target text is on the vertical axis. If a writer or translator completed a text by only moving forward, deleting all the typos immediately and never jumping back to previous parts of the text, the graph would be a straight line from the upper left corner to the lower right. In contrast to this idealized scenario, writers and translators often delete words, move parts of their emerging text to different locations, and insert new text in previously written sections. All of these revisions appear on the progression graph as dots that are linked to form a more or less jagged line, which reflects the course of the text production. 


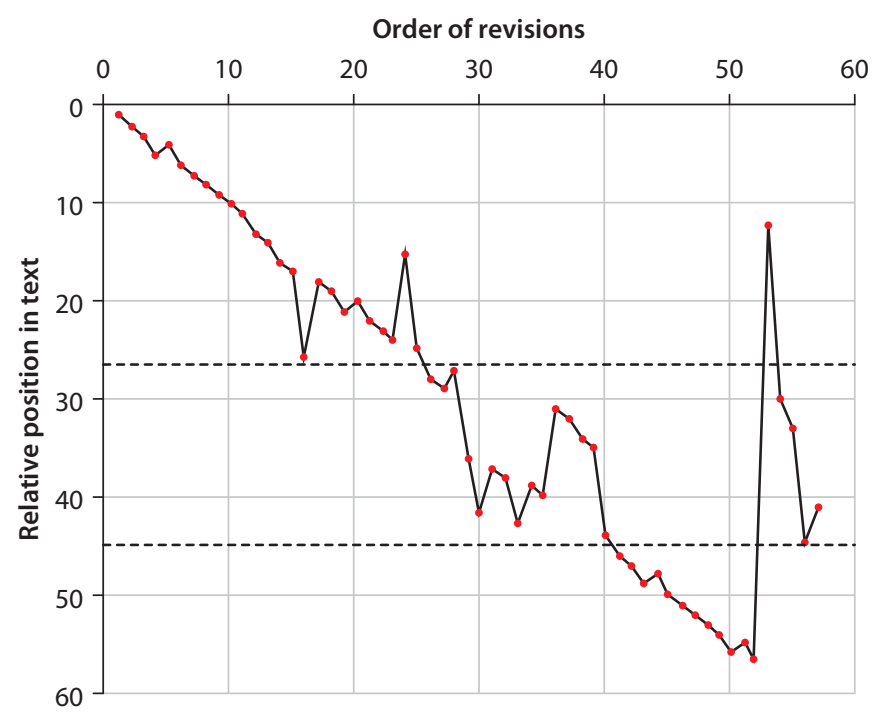

Figure 2. Progression graph of ET0413's translation process

The smoothness in translating the first part of the ST is apparent in the progression graph of ET0413's process (Figure 2). The revisions in the 5th sentence are delimited by the horizontal dotted lines: the uneven diagonal line in this section reflects how often the translator returned to an earlier part of the sentence to delete or insert text. The beginning of the revision phase of the translation process is obvious, since the line has a generally negative slope until the 53rd revision, which indicates a return to the beginning of the text. All of the subsequent revisions were made to the 5 th sentence.

\subsubsection{Eye tracking}

Progression analysis has recently been extended to include eye-tracking data when feasible. Such data complement the description of the writing or translation process by providing information about the focus of attention on the screen. This can be especially useful to understand the function of pauses in text production or in considerations of the role of reading in the writing or translation process. For example, the translator read the brief and the whole ST before starting her translation phase. She reread the introductory phrase of the 5th sentence before and during its translation, and then continually shifted her focus of attention between the 5th sentence of the ST and her emerging TT. She reread her TT sentence several times, fixating especially on the two time adverbials in it (see Figure 3). Finally, she reread the 5 th sentence of the ST two more times before moving on to the 6th sentence. 


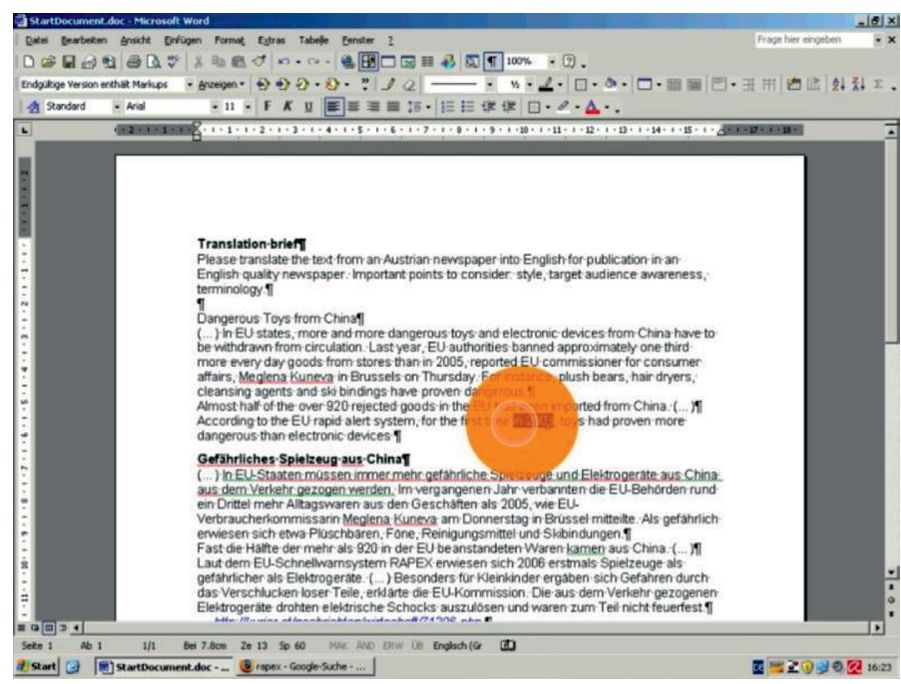

Figure 3. Sample fixation of time adverbials in ET0413's eye-tracking record

\subsection{Third level of progression analysis: awareness of decision-making}

The methods discussed above allow inferences to be made about the problems that the translator encountered and how she dealt with them but not about whether she was aware of what she was doing. When the translator viewed the screen recording of her process, she indicated in her retrospective verbalization that she was aware of the difficulty she had had with the time adverbials and attributed it to the fact that she was translating into her L2 (Table 2).

Table 2. Extracts from ET0413's RVP related to the production of the 5th sentence

\begin{tabular}{ll} 
Time & Comment \\
\hline $00: 11: 59$ & $\begin{array}{l}\text { here comes the part I actually thought about the most... which information should come where in the } \\
\text { English sentence. At the end I still wasn't really convinced of my solution and I changed it around at the } \\
\text { end. } \\
\text { ok, that is the first variant, then another one, a second and a third...I find it difficult to place all these } \\
\text { adverbials in English ... putting it at the beginning of the sentence might work with one but with two it } \\
\text { gets harder }\end{array}$ \\
and now I fiddled around with it again...yeah, I somehow have the feeling that a native speaker could say \\
this better... it probably wouldn't be a problem for them... putting things in the right order. This is \\
exactly the handicap I have as a non-native speaker.
\end{tabular}

The information from the cue-based RVP substantiates that the problem identified with the other methods was indeed a problem for the translator, that she seemed to understand what type of problem it was, and that she had constructed an explanation for the source of the problem (cf. Muñoz Martín 2010,181). This explanation was tested with version analysis of other TTs from the corpus that hers was drawn from, as described below. 


\subsection{Version analysis of translation products}

In our longitudinal study, various groups of translators have translated the same source texts under similar conditions, which makes a direct comparison of translation products possible with version analysis. The translation processes of the five volunteers and five randomly chosen processes recorded from their peers two years before were compared to those of nine freelance professionals who had translated the ST into their L1. In particular, the translation of the adjacent time adverbials in the 5th sentence of the ST was examined in detail (marked in bold and italics, respectively, in Table 3). Although no two versions were identical, most of the L2 translators either maintained the ST order of the two time adverbials but separated them with other sentence constituents or kept them adjacent but reversed the order and put them at the end of the sentence. Only one of them expressed the time information in sentence constituents other than adverbials (i.e., UE0310), a convincing solution except for the verb form.

The solutions by the professionals were not very different from those of the students: all of the patterns in the L2 translations also appeared in the L1 translations. However, there was more variety in the relative positions of the time adverbials in the L1 translations and more of a tendency to put the time information into other sentence constituents. This could reflect greater confidence on the part of the professionals in exploiting the linguistic resources of their L1 but it could also be due to their experience.

The participant whose process was examined in the previous section (ET0413) shared her solution to the time adverbials with three other students and three professionals. Interestingly, the variety of patterns in the version analysis of the two groups is very similar to the variety of versions of the sentence as ET0413 struggled

Table 3. Version analysis of translations by students (into L2) and professionals (into L1)

Translations of "erwiesen sich $\mathbf{2 0 0 6}$ erstmals Spielzeuge als gefährlicher als Elektrogeräte."

Translator

in 2006, toys proved for the first time to be more dangerous than electric appliances.

in 2006, toys were for the first time more dangerous than electrical appliances.

UE0315

in 2006 toys proved to be more harmful than electronic devices for the first time.

UE0321

In 2006, [...] proved toys to be more dangerous than electronic devices for the first time.

ב toys were in 2006 more dangerous than electrical appliances for the first time.

toys had proven more dangerous than electronic devices for the first time in $\mathbf{2 0 0 6}$. toys proved to be more dangerous than electrical appliances for the first time in 2006. certain toys have emerged to be more dangerous than electronical devices for the first time in 2006. toys were considered to be more dangerous than electronic devices for the first time in $\mathbf{2 0 0 6}$. ET0402 
with the positions of the time adverbials in her target text (Appendix B). Her solutions otherwise all matched the ST structure, whereas some of the other translators seemed more prepared to depart from the ST and to convey the information in different forms. In fact, the only student who did exactly that seemed to have little difficulty with this sentence (i.e., no revisions and no mention of it in the cue-based RVP). Other cue-based RVPs suggest that this could be a good strategy, as one of the professionals commented: "when a German sentence is turned the wrong way, and you don't know what else to do, I always use the verb to see" (Pro0709).

\section{Transferring research findings to training and back to the workplace}

Just as with newswriting processes, the different levels of progression analysis reveal information about reading processes, revision, research, consultation, and problem solving during the process of translation. Combining data from the three levels can help us identify when self-concepts associated with psychobiography, such as those concerning world knowledge or target language competence, may be putting undue strain on translators' cognitive resources. If a translation problem can be attributed to a lack of target-language competence, then a good strategy might be to exploit other linguistic or contextual resources, such as relying on other-revision in a text production chain rather than simply increasing the amount of self-revision (cf. Mossop 2007).

Progression analysis allows a more detailed examination of translation problems than most product analyses do, since each action performed on the computer can be reconstructed for every process or partial process. Version analyses can be used to determine how a particular translator or groups of translators deal with similar types of problems. With groups translating a single source text, various translations can be compared; in the workplace setting, when a ST is usually only translated once into each language, translations of STs with similar syntactic structures, terminology, genre conventions, and rhetorical purposes can be compared. Progression analysis can be applied in both settings and thus is suitable for use in experiments as well as in ethnographic investigations of communities of practice.

The insights gained from progression analysis about translation practices and translators' decision-making are being transferred into evidence-based translation training and coaching. In line with the results from transdisciplinary action research in related fields (cf. Bremner 2012; Jones and Stubbe 2004; Krohn 2008), we have found that students and professionals seem to profit from seeing not only what they and others do but also how they do it. The next step will be to apply the results of progression analysis towards optimizing work processes and conditions at the translation workplace. 


\section{References}

Archer, Margaret. 2003. Structure, Agency and the Internal Conversation. Cambridge: Cambridge University Press. DOI: 10.1017/CBO9781139087315

Bremner, Stephen. 2012. "Socialization and the Acquisition of Professional Discourse." Written Communication 29 (1): 7-32. DOI: 10.1177/0741088311424866

Ehrensberger-Dow, Maureen, and Alexander Künzli. 2010. "Methods of Accessing Metalinguistic Awareness: A Question of Quality?” In New Approaches in Translation Process Research [Copenhagen Studies in Language, 39], ed. by Susanne Göpferich, Fabio Alves, and Inger Mees, 113-132. Copenhagen: Samfundslitteratur.

Englund Dimitrova, Birgitta. 2005. Expertise and Explicitation in the Translation Process. Amsterdam: John Benjamins. DOI: 10.1075/btl.64

Hansen, Gyde. 2006. "Retrospection Methods in Translator Training and Translation Research." Journal of Specialised Translation 5: 2-41.

Jääskeläinen, Riitta. 2011. "Back to Basics: Designing a Study to Determine the Validity and the Reliability of Verbal Report Data on Translation Processes." In Cognitive Explorations of Translation, ed. by Sharon O'Brien, 15-29. London: Continuum.

Jacobs, Geert. 2011. "Press Conferences on the Internet." Journal of Pragmatics 43 (7): 19001911. DOI: $10.1016 /$ j.pragma.2010.09.019

Jakobsen, Arnt L. 2006. "Research Methods in Translation - Translog." In Computer KeyStroke Logging and Writing: Methods and Applications, ed. by Kirk P. H. Sullivan, and Eva Lindgren, 95-105. Oxford: Elsevier.

Jones, Deborah, and Maria Stubbe. 2004. "Communication and the Reflective Practitioner. A Shared Perspective from Sociolinguistics and Organisational Communication." International Journal of Applied Linguistics 14 (2): 185-211.

DOI: $10.1111 /$ j.1473-4192.2004.00059.x

Juzwik, Mary M., Svjetlana Curcic, Kimberly Wolbers, Kathleen D. Moxley, Lisa M. Dimling, and Rebecca K. Shankland. 2006. "Writing into the 21st Century: An Overview of Research on Writing, 1999 to 2004.” Written Communication 23 (4): 451-476.

DOI: $10.1177 / 0741088306291619$

Kollberg, Py. 1998. S-Notation - A Computer-Based Method for Studying and Representing Text Composition. Licentiate thesis, Royal Institute of Technology, IPLab, Stockholm.

Kollberg, Py, and Kerstin Severinson Eklundh. 2001. "Analyzing Writers' Revising Patterns with S-Notation Analysis." In Contemporary Tools and Techniques for Studying Writing, ed. by Thierry Olive, and C. Michael Levy, 89-104. Dordrecht: Kluwer Academic Publishers.

Krohn, Wolfgang. 2008. "Learning from Case Studies." In Handbook of Transdisciplinary Research, ed. by Holger Hoffmann-Riem et al, 369-383. Berlin: Springer.

DOI: 10.1007/978-1-4020-6699-3_24

Lee-Jahnke, Hannelore. 2005. "New Cognitive Approaches in Process-Oriented Translation Training." Meta 50 (2): 359-377. DOI: 10.7202/010942ar

Levy, C. Michael, J. Pamela Marek, and Joseph Lea. 1996. "Concurrent and Retrospective Protocols in Writing Research." In Theories, Models and Methodology in Writing Research, ed. by Gert Rijlaarsdam, Huub Van den Bergh, and Michael Couzijn, 542-556. Amsterdam: Amsterdam University Press.

Massey, Gary, and Maureen Ehrensberger-Dow. 2010. "Investigating Demands on Language । Professionals.” Bulletin suisse de linguistique appliquée (Special Issue 1): 127-141. 
Mossop, Brian. 2007. "Empirical Studies of Revision: What We Know and Need to Know." The Journal of Specialised Translation 8: 5-20.

Muñoz Martín, Ricardo. 2010. “On Paradigms and Cognitive Translatology.” In Translation and Cognition, ed. by Gregory Shreve, and Erik Angelone, 169-187. Amsterdam: John Benjamins. DOI: 10.1075/ata.xv.10mun

Perrin, Daniel. 2003. "Progression Analysis (PA). Investigating Writing Strategies at the Workplace." Journal of Pragmatics 35 (6): 907-921. DOI: 10.1016/S0378-2166(02)00125-X

Perrin, Daniel. 2006. "Progression Analysis: An Ethnographic, Computer-Based Multi-Method Approach to Investigate Natural Writing Processes." In Writing and Digital Media, ed. by Luuk Van Waes, Mariëlle Leijten, and Christine M. Neuwirth, 173-179. Oxford: Elsevier.

Perrin, Daniel. 2013. The Linguistics of Newswriting. Amsterdam: John Benjamins. DOI: $10.1075 /$ aals.11

Perrin, Daniel, and Maureen Ehrensberger-Dow. 2008. "Media Competence." In Handbook of Communicative Competence, ed. by Gert Rickheit, and Hans Strohner, 277-312. New York: de Gruyter.

Rijlaarsdam, Gert, Huub Van den Bergh, and Michael Couzijn (eds). 1996. Theories, Models and Methodology in Writing Research. Amsterdam: Amsterdam University Press.

DOI: $10.5117 / 9789053561973$

Tashakkori, Abbas, and Charles Teddlie. 2003. "Major Issues and Controversies in the Use of Mixed Methods in Social and Behavioral Research." In Handbook of Mixed Methods in Social and Behavioral Research, ed. by Abbas Tashakkori, and Charles Teddlie, 3-50. Thousand Oaks, CA: Sage.

Van Hout, Tom, Henk Pander Maat, and Wim De Preter. 2011. "Writing from News Sources. The Case of Apple TV." Journal of Pragmatics 43 (7): 1876-1889.

DOI: 10.1016/j.pragma.2010.09.024

Van Waes, Luuk, and Mariëlle Leijten. 2006. "Logging Writing Processes with Inputlog." In Writing and Digital Media, ed. by Luuk Van Waes, Mariëlle Leijten, and Christine M. Neuwirth, 158-165. Oxford: Elsevier. DOI: 10.1163/9781849508209

\section{Appendix A. Translation brief and German source text}

\section{Translation brief}

Please translate the text from an Austrian newspaper into English for publication in an English quality newspaper. Important points to consider: style, target audience awareness, terminology.

\section{Gefährliches Spielzeug aus China}

(...) In EU-Staaten müssen immer mehr gefährliche Spielzeuge und Elektrogeräte aus China aus dem Verkehr gezogen werden. Im vergangenen Jahr verbannten die EU-Behörden rund ein Drittel mehr Alltagswaren aus den Geschäften als 2005, wie EU-Verbraucherkommissarin Meglena Kuneva am Donnerstag in Brüssel mitteilte. Als gefährlich erwiesen sich etwa Plüschbären, Föne, Reinigungsmittel und Skibindungen.

Fast die Hälfte der mehr als 920 in der EU beanstandeten Waren kamen aus China. (...)

Laut dem EU-Schnellwarnsystem RAPEX erwiesen sich 2006 erstmals Spielzeuge als gefährlicher als Elektrogeräte. (...) Besonders für Kleinkinder ergäben sich Gefahren durch das Verschlucken loser Teile, erklärte die EU-Kommission. Die aus dem Verkehr gezogenen Elektrogeräte drohten elektrische Schocks auszulösen und waren zum Teil nicht feuerfest. 


\section{Appendix B. Steps in ET0413's production of the 5th sentence of the target text}

\begin{tabular}{|c|c|c|}
\hline Start time & Action & Sentence version \\
\hline $00: 11: 21$ & writes & According to the rapid alers \\
\hline $00: 11: 45$ & backspaces & According to the rapid alers \\
\hline $00: 11: 45$ & writes & According to the rapid alert si \\
\hline $00: 11: 46$ & backspaces & According to the rapid alert si \\
\hline $00: 11: 46$ & writes & According to the rapid alert system \\
\hline $00: 11: 53$ & $\underline{\text { inserts }}$ & According to the EU rapid alert system \\
\hline $00: 11: 58$ & $\overline{\text { writes }}$ & According to the $\overline{\mathrm{EU}}$ rapid alert system, 2006 \\
\hline $00: 12: 06$ & backspaces & According to the EU rapid alert system, 2006 \\
\hline $00: 12: 12$ & writes & According to the EU rapid alert system, in 2006 , toys hav \\
\hline $00: 12: 20$ & backspaces & According to the EU rapid alert system, in 2006 , toys ha \\
\hline $00: 12: 21$ & writes & $\begin{array}{l}\text { According to the EU rapid alert system, in } 2006 \text {, toys had proven more dangerous } \\
\text { than electronic devices }\end{array}$ \\
\hline $00: 12: 37$ & $\underline{\text { inserts }}$ & $\begin{array}{l}\text { According to the EU rapid alert system, for the first time in } 2006 \text {, toys had proven } \\
\text { more dangerous than electronic devices }\end{array}$ \\
\hline $00: 12: 54$ & euts & $\begin{array}{l}\text { According to the EU rapid alert system, for the first time } 2006 \text {, toys had proven } \\
\text { more dangerous than electronic devices }\end{array}$ \\
\hline $00: 12: 55$ & pastes & $\begin{array}{l}\text { According to the EU rapid alert system, for the first time, toys had proven more } \\
\text { dangerous than electronic devices in } 2006\end{array}$ \\
\hline $00: 13: 12$ & writes & $\begin{array}{l}\text { According to the EU rapid alert system, for the first time, toys had proven more } \\
\text { dangerous than electronic devices in } 2006 \text {. }\end{array}$ \\
\hline $00: 13: 14$ & euts & $\begin{array}{l}\text { According to the EU rapid alert system, for the first time, toys had proven more } \\
\text { dangerous than electronic devices in } 2006 \text {. }\end{array}$ \\
\hline $00: 13: 15$ & pastes & $\begin{array}{l}\text { According to the EU rapid alert system, for the first time in } 2006 \text {, toys had proven } \\
\text { more dangerous than electronic devices. }\end{array}$ \\
\hline $00: 13: 20$ & deletes & $\begin{array}{l}\text { According to the EU rapid alert system, for the first time in } 2006 \text {, toys had proven } \\
\text { more dangerous than electronic devices. }\end{array}$ \\
\hline $00: 13: 22$ & $\underline{\text { inserts }}$ & $\begin{array}{l}\text { According to the EU rapid alert system, in } 2006 \text { for the first time, toys had proven } \\
\text { more dangerous than electronic devices. }\end{array}$ \\
\hline $00: 13: 23$ & ents & $\begin{array}{l}\text { According to the EU rapid alert system, in } 2006 \text { for the first tome, toys had proven } \\
\text { more dangerous than electronic devices. }\end{array}$ \\
\hline $00: 13: 27$ & pastes & $\begin{array}{l}\text { According to the EU rapid alert system, in } 2006 \text {, toys had proven more dangerous } \\
\text { than electronic devices for the first time. }\end{array}$ \\
\hline & & $\ldots$ (continues translating rest of source text) \\
\hline $00: 18: 42$ & $\underline{\text { inserts }}$ & $\begin{array}{l}\text { According to the EU rapid alert system RAPEX, in } 2006 \text {, toys had proven more } \\
\text { dangerous than electronic devices for the first time. }\end{array}$ \\
\hline $00: 18: 48$ & euts & $\begin{array}{l}\text { According to the EU rapid alert system RAPEX, in } 2006 \text {, toys had proven more } \\
\text { dangerous than electronic devices for the first time. }\end{array}$ \\
\hline $00: 18: 50$ & pastes & $\begin{array}{l}\text { According to the EU rapid alert system RAPEX, , toys had proven more dangerous } \\
\text { than electronic devices for the first time in } 2006 .\end{array}$ \\
\hline $00: 18: 54$ & deletes & $\begin{array}{l}\text { According to the EU rapid alert system RAPEX, }- \text {, toys had proven more dangerous } \\
\text { than electronic devices for the first time in } 2006 \text {. }\end{array}$ \\
\hline
\end{tabular}




\title{
Metaphor in translation
}

\section{Possibilities for process research}

\author{
Christina Schäffner and Mark Shuttleworth \\ Aston University, UK / University College London, UK
}

\begin{abstract}
This chapter explores potential benefits of closer interaction between metaphor studies and translation process research. It presents some developments within translation studies that make use of conceptual metaphor theory and illustrates some process research methods for investigating metaphors. The chapter considers a number of methodological recommendations and argues that the need to take full account of insights from metaphor studies and associated disciplines is of greatest importance. Another significant potential innovation is the use of a multilingual approach in respect of both product- and process-oriented studies in order to increase both the amount and the generality of data available for analysis. Thirdly, it is important to extend the current source-text (ST) oriented approach. The chapter concludes by suggesting some options for triangulating data gathered through a combination of methods.
\end{abstract}

Keywords: metaphor, metaphor studies, translation process research, TAPs, keystroke logging, eye tracking, multilingual approach, triangulation

\section{Introduction}

There are many methodological approaches that could profitably be implemented as part of a comprehensive study of metaphor in translation (MiT) within the paradigms of process research. While a number of these are derived from tendencies already existing elsewhere in translation studies, some are innovative and as yet virtually untried. In addition, some quite clearly also possess a wider potential application within the discipline.

Section 2 of this chapter focuses on two areas: the interlinking of MiT research with the discipline of metaphor studies and the use of a multilingual approach. Section 3 reviews some of the translation process studies that have investigated metaphors. It presents the main aims of such studies, the methods used and the 
most significant findings, and then reflects on how they can be extended to enhance our understanding of MiT and how such findings can be made useful to metaphor studies.

\section{Theoretical approaches}

\subsection{Interaction with metaphor studies}

Of greatest importance is the need to take full account of insights from metaphor studies. Although it is by no means the only theoretical framework in existence, research into metaphor has been largely dominated by the conceptual metaphor approach since it was first proposed (in its current form) in Lakoff and Johnson (1980). According to those who subscribe to this approach, a metaphor can be thought of not as an isolated figurative expression in a text, but as a 'mapping' from one domain of experience to another - a kind of mental connection made between two unrelated concepts or areas of experience that allows one to think and talk about one of these concepts or areas in terms usually reserved for the other and that potentially sanctions an open-ended number of individual 'metaphorical expressions' relating to that particular area (Lakoff 1993, 203; Evans and Green 2006, 295). (Examples of mappings would include ARGUMENT IS WAR, GOOD IS UP or COMPUTERS ARE HUMANS). ${ }^{1}$ This approach does not perhaps offer the best theoretical framework for the study of MiT because of its tendency to play down the amount of interlingual variation in metaphorical patterns that is caused by linguistic or cultural factors. In spite of this, it is probably the one that has exerted the strongest influence on MiT researchers - albeit in a slightly adapted form - although some research in this area situates the theoretical centre of gravity exclusively within translation studies or incorporates theoretical metaphor studies concepts in a fairly circumscribed manner. Because of its emphasis on the psychological rather than textual aspects of metaphor and the insights that it offers into the brain's cognitive processes, the conceptual metaphor approach's applicability within process research should be clear.

Not surprisingly, metaphor scholars use a wide range of theoretical concepts, categories and parameters for the purposes of identifying, classifying and describing metaphor. To quote one example, Cameron discusses a total of nine "graded descriptors of metaphor" (1999, 123): incongruity, novelty/conventionality, attitudinal impact, explication, familiarity, cognitive demand, explicitness, connotative power and systematicity $(1999,124-30)$. Parameters such as these, either

1. In this article we use small capitals to indicate mappings. 
combined with others from within translation studies or used in isolation, can form the basis for profoundly probing investigations of MiT. This is an approach that has been fruitfully adopted, for example, by Al-Harrasi (2001), who has used it to present a list of translation procedures that is significantly different from any previously produced, as it is based on the theoretical categories of conceptual metaphor theory $(2001,277-88)$. Unfortunately, space does not permit a detailed discussion of his proposals. Suffice it to say, however, that although not entirely above criticism, as far as we are aware his list of procedures is the first attempt to produce a detailed alternative taxonomy based on metaphor theory, and one that opens up a whole new possible direction for research.

The list of parameters drawn from metaphor studies and cognitive linguistics that can be explored in this respect is long and offers much potential for translation studies research. The work of one of the present authors, for example, focuses on a total of seven parameters: mapping, typological class, purpose, level of categorisation (a single concept that encompasses class inclusion and degree of specificity), metaphor type (whether it is a conceptual or image metaphor), metaphor provenance (whether it is image-schematic or based on propositional knowledge) and conventionality (see Shuttleworth 2011). One of the topics that this research aims to investigate is the significance that each of these parameters may possess in terms of its possible influence on translators' decisions. While the sixth parameter, that of metaphor provenance, focuses on an area similar to that studied by Al-Harrasi, many of the others break new ground in that to our knowledge they have not hitherto been used as a basis for MiT research. More results are of course hoped for, although one interesting initial finding is that it seems very likely that a metaphorical expression's typological class - i.e., whether it is an example of concretisation, humanisation, dehumanisation or whatever - reflects much clearer tendencies of translator behaviour than the particular mapping from which it derives. While some initial work is now underway in this area, much remains to be done.

Throughout, the questions to be asked should be 1) whether a particular parameter can be used to produce an innovative or insightful list of translation procedures, 2) the extent to which such parameters might exert an influence on translators' decision-making and - reflecting back on metaphor studies - 3) whether the answers to the previous two questions serve to confirm, qualify or perhaps invalidate the theoretical or psychological significance of a particular concept or parameter. This last point is important: as translation studies is an 'interdiscipline, there has (quite rightly) been much discussion about utilising the concepts and approaches of other disciplines for our own purposes. Now that translation studies has come of age and established its true identity, however, it 
is in our view very important that we should be able to offer back to these other disciplines insights and findings of our own, in the hope that they may be of value.

\subsection{Use of a multilingual approach}

Another important potential innovation - and one that is not only applicable to MiT, of course - is the use of a multilingual approach to increase both the number and generality of examples available for analysis. As we see it, the advantage of using multilingual corpora is that it enables the researcher to focus (for want of a better word) on more 'universal' aspects of translator behaviour. By this we do not necessarily mean the 'universals of translation' that have been widely discussed (see, for example, Mauranen and Kujamäki 2004; Olohan 2004), but simply MiT tendencies that may transcend the norms and constraints of a single translation context (to the extent to which this is possible) and that hopefully therefore provide us with a more generalised idea of translator behaviour within this specific area.

What is meant by 'multilingual' is that translations of a single ST or group of STs into a range of different languages can be studied alongside each other. It has already been implemented in one of the present authors' text-based investigations, which involves a corpus consisting of Scientific American articles along with their official published translations into French, Italian, German, Russian and Polish (see Shuttleworth 2011), although to our knowledge it has not yet been widely utilised in process research.

When translations into more than one target language (TL) are compared, some interesting results can emerge, and can hopefully be seen in the following two brief examples, both of which relate to expressions instantiating the mapping NATURE IS A CREATOR OF CODE.

In the first example, we see how a weak ST metaphor is removed in both TLs: ${ }^{2}$

(1) English: only 100 of one million alternatives had a lower error value than nature's code

Russian: оолько у 100 альтернативных кодов из миллиона цена ошибки меньше, чем у канонического

[only in 100 alternative codes is the price of an error lower than in the canonical one]

Italian: $\quad$ solo 100 su un milione erano migliori di quello naturale [only 100 out of a million were better than the natural one]

2. Bold typeface indicates metaphorical expressions and underlining highlights non-metaphorical translations of ST metaphors. 
The ST metaphor is one of humanisation, and is generated purely by the use of the possessive construction (nature's code): convert it to another construction (the natural code, for example) and the (albeit weak) entailment that nature has created this genetic code vanishes. The fact that this happens in numerous examples across different TLs (German, Polish and French as well as Russian and Italian) is certainly worthy of note. What is also interesting, however, is that in the Russian translation the wording opted for appears to be just as unusual for its TL as a more literal rendering would have been.

The second example also involves a fairly marginal metaphor. In this instance, however, the metaphor is not only retained in both TLs, but is greatly intensified:

(2) English: Nature's version [of the genetic code] looked less elegant than several of the theorists' hypotheses.

German: Die Lösung, welche die Natur gefunden hatte, erschien weniger elegant als einige theoretische Vorschläge.

[The solution that nature had found appeared less elegant than a number of theoretical proposals.]

Russian: То, что придумала природа, выглядело гораздо менее изобретательным, чем многие гипотезы ученых.

[What nature thought up seemed much less inventive than many scientists' hypotheses.]

(The same thing occurs, but to a lesser extent, in Polish, French and Italian.) What is striking here is that all the translators see fit to avoid a direct equivalent of the seemingly neutral ST phrase - once again, an interesting piece of translator behaviour.

Due attention does of course still need to be paid to translation norms specific to particular language pairs, although one of the main advantages of such a datarich multilingual study is that it can potentially produce results that allow one to draw conclusions at a much higher level of generalisation. When using this approach it is of course important to ensure not only cross-linguistic comparability, but also comparability in terms of the translators' levels of competence.

\section{Translation process studies and metaphor}

Most of the work conducted on MiT within translation studies has been textbased, and thus product-oriented. The text shows us the result of very complex cognitive processes, which moreover occurred in specific socio-cultural, historical, and institutional contexts. The interaction of all these aspects would need to be taken into account in linking textual profiles to the factors that are decisive in the 
translators' decision making. As has repeatedly been said, when we have only the translation product available for analysis, we can at best speculate about the cognitive processes that led up to it. However, we cannot retrace the actual pathways of the translator's decision-making procedures. Investigations into translation processes conducted so far have tried to fill this gap in our knowledge. Methods used in order to get insights into actual cognitive processes have included think-aloud protocol studies (TAPs), and more recently keystroke logging and eye tracking. Only very few studies were exclusively devoted to investigating cognitive processes in respect of MiT. In this section, we will present some of this research, focusing on the aims and findings, and review the researchers' explanations. We will conclude with some suggestions for expanding process studies for MiT research.

\subsection{Addressing metaphors via TAPs}

TAP-based research has been employed, for example, to test the Cognitive Translation Hypothesis, which states that "metaphorical expressions take more time and are more difficult to translate if they exploit a different cognitive domain than the target language equivalent expression" (Tirkkonen-Condit 2001, 11). Martikainen (1999) and Tirkkonen-Condit (2001; 2002) use professional translators in simulated translation tasks, measuring time and the length of TAP segments, counting the lines of target text (TT) produced, and also asking the translators to comment on their own satisfaction with the translations they produced. Their research confirms the hypothesis. For example, be out of one's depth, an idiomatic expression with the semantic content of 'not understanding' that exploits the cognitive domain of vertical dimension does not have a Finnish idiomatic expression that exploits the same cognitive domain. Consequently, translating this metaphorical expression takes longer since translators have to search for another conceptual domain. Metaphorical expressions with different domains also result in more verbalisation in the TAPs and in more translation solutions. The overall conclusion is that delay in the translation process, and the related uncertainty in the translation of different domain metaphors, is evidence of concept mediation.

Kussmaul uses dialogue protocols of students, in particular with reference to creativity research. One of the examples he discusses is the debate concerning the translation into German of the sentence 'He began to make progress in becoming a human being instead of a RUBBISH BIN', taken from a psychology self-help book. The students initially verbalised more direct German equivalents to "rubbish bin": "Mülleimer", "Abfalleimer", but then resorted to using verbal phrases in their reflections (“... statt dass alle auf ihm rumtrampeln", i.e., instead of others trampling on him). This verbal description of an action is turned into a noun again 
by another student, "Fußabtreter" (doormat). Making use of scenes-and-frames semantics, Kussmaul (2000) argues that words and phrases (frames) activate mental images (scenes) and that the dialogue protocol shows that a scene is verbalised by using metaphorical expressions. The metaphor in the ST ("rubbish bin") is interpreted as a metaphorical image (an object of disrespect), which leads to elaborating on actions associated with disrespect (trampling on a person), which finally leads to the verbalisation of another object of disrespect ("Fußabtreter"). Kussmaul argues that the metaphor in the ST has led to a new metaphor in the TT via chaining of metaphorical scenes, and that the two different metaphors are thus interconnected via a scene and metaphorical images.

Similarly to Tirkkonen-Condit, his dialogue protocols too reflect the idea that thought processes are happening at conceptual levels, although they are often triggered by metaphorical expressions and the initial attempt to provide a TL equivalent for a linguistic metaphor. This seems to lend support to Göpferich's hypothetical model of processes of metaphor translation. In her model, Göpferich $(2008,203)$ distinguishes an analytical phase and a synthesis phase. The analytical phase consists of metaphor identification, metaphor interpretation, and specifying the function of the metaphor in the text. In the synthesis phase, translators need to decide on the most appropriate translation procedure (e.g., literal translation, substitution by a different metaphor, paraphrase) and the actual verbalisation in the TT. She acknowledges that this model needs to be tested by empirical research $(2008,202)$.

Kussmaul's dialogue protocols of student performance do not always provide evidence that they immediately identified an expression as a metaphorical one. Tirkkonen-Condit $(2002,103)$ too asked whether experts access the conceptual level more or less frequently than novice translators. What is also not yet clear is what exactly triggers the need to access the conceptual level in real translation events.

\subsection{Addressing metaphors via keystroke logging}

Using the keystroke logging software Translog, Jakobsen, Jensen, and Mees (2007) measured processing time and noticed that idiomatic expressions (which are very often metaphorical) slow down the translation process. This was generally the same for expert translators and students although expert translators worked in longer segments than students. This again seems to lend support to the Cognitive Translation Hypothesis. Keystroke logging has also been combined with thinkaloud data and/or with retrospective interviews. Kussmaul's experimental study on creativity, which combined Translog with retrospective interviews, is interesting for MiT as well (Kussmaul 2003). Professional translators had been asked to 
translate a text on the topic of European integration into German. One paragraph was particularly rich in metaphors:

Britain cannot accept a Europe dominated by the self-interest of a Franco-German alliance. Nor do we need to accept a looser concept: a TWO-TIER COMMUNITY, divided between the PACESETTERS and the rest. It is quite obvious who the PACESETTERS are determined to be. It is equally obvious that, while they may accept us as partners, they will never allow us TO IMPOSE A BRAKE on their ambition. (Kussmaul 2003, 133; capitalisation added for emphasis)

The four translators arrived at significantly different solutions. The retrospective interviews relating to their Translog reports indicate that they were fully aware of the metaphors in the text. One translator had rendered the metaphorical expressions underlined above as "deutsch-französische Lokomotive" (German-French engine), "Führungsrolle" (leading role) and "ohne wirkliches Mitsprache- und Vetorecht" (without any real right of co-determination or veto). When confronted with the Translog report, she commented that the decision to use "deutsch-französische Lokomotive" was motivated by its frequent use in the German press, and that "Lokomotive" fitted well with "pacesetter". Since she wanted to avoid lexical repetition she decided on "Führungsrolle" in the following sentence. The Translog report records a pause of 28 seconds before the translator started typing her TT for the last sentence. She commented that she had associated "brake" with stopping, which a veto would achieve as well. At the surface level, the TT produced looks less metaphorical than the ST, since only "Lokomotive" could easily be characterised as a metaphorical expression. Kussmaul speaks of demetaphorisation for translating "impose a brake" as "Vetorecht", but refers to cognitive chaining between the domains of train journey and politics. We can also say that the TT is structured around the conceptual metaphor POLITICS IS A JOURNEY, and even more specifically, POLITICS IS A TRAIN JOURNEY. The knowledge of train journeys is mapped onto politics as a journey, with the common element being that journeys can be stopped, by putting on a brake for trains, and using a veto in politics.

Another translator had opted for "Europa der zwei Geschwindigkeiten" (twospeed Europe), "Schrittmacher" (pacesetters), and "als Bremser dabei haben" (have present as brakesmen), thus also structuring the TT coherently around a conceptual metaphor POLITICS IS A JOURNEY, albeit without specifying this journey as a train journey. The Translog report records a 3-minute pause before the text for this extract is written. Faced with this Translog report, the translator criticised the ST for including two incompatible metaphorical images and argued that for his TT he opted for one coherent metaphorical domain. Kussmaul comments that the translator activated a metaphorical scene of a journey and that individual elements of this scene are lexicalised in the TT, also reflected in the translation of 
"partners" as "Mitfahrer" (fellow travellers). At the surface level, this TT has even more metaphorical expressions than the ST, although Kussmaul does not explicitly comment on this feature. His conclusion is that both TTs reflect more creative solutions than the TTs produced by the other two translators. As the main reason for this creativity he identifies the activation of world knowledge and related depth of reflection, as evidenced in the pauses, total length of time for TT production and retrospective comments.

In respect of the question if and when translators actually access the conceptual level, the data gathered from Translog records and retrospective interviews are not conclusive. Translators explained their decisions with reference to their awareness of structures used in the press, with reference to the translation skopos, intratextual coherence, and stylistic considerations. Although based exclusively on TAPs, Tirkkonen-Condit $(2002,112)$ too had noticed that the translators often searched for metaphorical expressions and conceptual domains that would be compatible with the overall vision they have of the emerging TT. These results also illustrate that the factors that influence the decisions for dealing with metaphors are manifold.

\subsection{Addressing metaphors via eye tracking}

The use of eye tracking is based on the assumption that there is a correlation between the time readers fixate on a word and the amount of processing that takes place. Of particular interest for MiT are the experiments conducted by Sjørup $(2008$; 2011). Her hypothesis is that "translators dwell longer over the processing complexities involved in translating a metaphor than a non-metaphorical concept" (Sjørup 2008, 53). Using naturally occurring text and professional translators she studied eye fixation time and discovered that there was indeed a longer fixation time for metaphors compared to non-metaphorical language. This finding seemed to confirm her hypothesis. However, she also argues that it is "impossible to distinguish the comprehension aspect from the text production task" (Sjørup $2008,68)$. Although longer fixation times were noticed and can be interpreted as a greater cognitive processing load, it is impossible to determine how this load is distributed between metaphor interpretation and the choice of a translation strategy and a TT formulation.

In her later study of 2011, she combined the analysis of gaze time with an investigation of translation strategies used. She complemented her hypothesis by the assumption that "the choice of translation strategy would have an effect on the cognitive effort involved in metaphor translation" (Sjørup 2011, 201). However, she distinguished only three translation strategies: use of direct metaphorical equivalent, use of another metaphorical phrase, and paraphrasing. Moreover, she hypothesised that using a direct metaphorical equivalent would be the preferred 
strategy and that paraphrasing would require most cognitive effort. In her study, professional translators translated two texts involving different conceptual domains. Although the distribution of the three translation strategies differed in the two texts, Sjørup did indeed identify a link between the translation strategy and the gaze time. She comments that the higher cognitive load for paraphrase as a translation strategy is due to two shifts involved: a shift from one domain to another, and a shift for metaphorical expressions to literal ones. However, the longer gaze time could also be related to a translator reflecting on consequences of local decisions for the overall function of the text - a point not problematised in depth by Sjørup.

\subsection{Main insights and new questions}

What process research using verbal data, keystroke log data and eye-tracking data has revealed is that metaphors do indeed seem to be linked to greater cognitive load. This is illustrated in longer pauses, total length of completing the translation task and more uncertainty (verbalised in TAPs and/or noticeable in Translog reports). Although the research illustrated above makes use of conceptual metaphor theory, the analyses focus on metaphorical expressions (cf. Kussmaul's description of rendering "impose a brake" as "Vetorecht" as demetaphorisation). In speaking of translation strategies, scholars still tend to refer to the types which were set up on the basis of a more traditional understanding of metaphor as a word reflecting a non-literal meaning. The TTs produced could be reinvestigated by making use of translation strategies that were developed on the basis of conceptual metaphor theory (such as those in Al-Harrasi 2001 or Schäffner 2004). Although these new types of strategy were suggested purely on the basis of product analyses (comparisons of STs and TTs), a re-evaluation of TAPs could test their validity. Scholars who used TAPs and retrospective interviews have argued that their data have not been conclusive in arriving at a firm evaluation of whether the translators did indeed access the conceptual level or whether they were guided by the surface structure. One reason for this conclusion could be that although they made use of conceptual metaphor theory in commenting on scene activation and chaining, they still resorted to translation strategies which were focused on surface structures.

Research into MiT can also benefit enormously from combining research methods, such as bringing together data gained from introspection (TAPs) and data generated by keystroke logging and/or data gathered from retrospective interviews as complementary sets to study one and the same translation event. These three methods were already combined by Sjørup, although her 2011 chapter reports only on the eye-tracking data. Key-logging data can show whether translators initially typed the closest TL equivalent and changed it at a later stage, thus lending support (or not) to the assumption that replacing a metaphor by an 
equivalent metaphor is the default strategy. The study of the position of pauses, before typing or after typing and/or correction, may also give more insights into the question about distribution load between metaphor interpretation and TT formulation. TAPs and/or retrospective interviews related to key-logging data and/or eye-tracking data could then provide additional input into the analyses. All scholars who have used various methods have pointed out that it is dangerous to make sweeping generalisations about processes in metaphor translation. They have discovered that subjects tend to differ enormously in their performance, which just confirms that there are several variables in the translation processes. A combination of methods and data sets, in short: triangulation, can thus lend more weight to hypotheses and explanations (as has been shown by Alves et al. 2010, albeit in investigating grammatical metaphors).

Metaphor researchers have also realised that there is both cross-cultural similarity and variation in metaphor. Variation may be reflected in the fact that various languages have different linguistic expressions for the same conceptual metaphor (Kövecses 2005). In a study using corpus data, Deignan, Gabrys, and Solska (1997) identified a number of cross-linguistic differences between conceptual metaphors and their linguistic variations which they classified into four types: same conceptual metaphor and equivalent linguistic expression; same conceptual metaphor but different linguistic expressions; different conceptual metaphors used in the two languages; and words and expressions with the same literal meanings but different metaphorical meanings. One possibility for translation studies to contribute to this investigation would be the multilingual approach, already mentioned above. Product-oriented analyses of multilingual texts such as those illustrated in 2.2, however, would also need to take into account that in all probability these texts were not produced in a truly parallel way but that their final structure was also determined by additional processes in their respective socio-cultural and institutional contexts (especially editing). Process studies could bring more insights if the multilingual texts were produced in identical conditions. For example, a number of translators could be asked to translate the same ST into several TLs, all translators working at the same time in the same setting, using the same methods, ideally in combination (e.g., keystroke logging and/or eye fixation and/or TAPs and/or retrospective interviews). Analysing the data gained in this way should not only give us more insights into cross-cultural variation of metaphors and metaphorical expressions, operations at the lexical or cognitive level, and comprehension vs. formulation load, but also identify subjective variables (e.g., background, training and habitus). 


\section{Conclusion}

This chapter has looked at a number of possible suggestions for new directions of research into MiT. New research would add significantly to existing research in both translation studies and metaphor studies, which so far have worked mainly in parallel. As we have illustrated, research into MiT in translation studies has been mainly product-oriented, identifying metaphorical expressions in STs and the translation strategies in the TTs. Incidentally, there has been more research on metaphors in translation than in interpreting. Translation process research has mainly focused on the cognitive load involved in dealing with metaphors, as identified in processing differences with respect to time, eye gaze or insecurity in TT production. Although in the analysis of processes scholars did refer to conceptual metaphor theories, they did not (yet) problematise these theories. Moreover, in speaking about translation strategies, they tended to focus on linguistic expressions and more traditional strategies. Process research could also be used to test alternative types of translation strategies as well as the descriptors and parameters of metaphors as suggested in 2.1. Such testing could be done by revisiting TAPs recorded in earlier research and re-analysing them from a different perspective and/or with a different aim.

As said above, research going on in metaphor studies does include an investigation into cross-cultural similarity and variation in metaphor, but authentic translations have hardly ever been used as input for such analyses. Translation process studies could thus also provide authentic data to verify (or falsify) hypotheses about cross-linguistic differences in conceptual metaphors and linguistic expressions sanctioned by them. The use of multilingual corpora for such studies seems particularly promising.

Many of the proposals contained in this chapter can be adapted to MiT research without significant difficulty. It is believed that implementing at least some of them will significantly extend the reach of translation studies research into this fascinating but highly complex subject. A closer interaction between translation process studies and metaphor studies can moreover lead to deeper insights and added value, since, as van Dijk argued some years ago in reflecting on interdisciplinarity, "most theoretical renewal takes place at the boundaries between several disciplines" (van Dijk 1995, 460). 


\section{References}

Al-Harrasi, Abdulla. 2001. Metaphor in (Arabic-into-English) Translation with Specific Reference to Metaphorical Concepts and Expressions in Political Discourse. Unpublished PhD thesis, Aston University.

Alves, Fabio, Adriana Pagano, Stella Neumann, Erich Steiner, and Silvia Hansen-Schirra. 2010. "Translation Units and Grammatical Shifts. Towards an Integration of Product and ProcessBased Translation Research.” In Translation and Cognition, ed. by Gregory M. Shreve, and Erik Anglone, 109-141. Amsterdam: John Benjamins. DOI: 10.1075/ata.xv.07alv

Cameron, Lynne. 1999. "Identifying and Describing Metaphor in Spoken Discourse." In Researching and Applying Metaphor, ed. by Lynne Cameron, and Graham Low, 105-132. New York: Cambridge University Press. DOI: 10.1017/CBO9781139524704.009

Deignan, Alice, Danuta Gabrys, and Agniezska Solska. 1997. "Teaching English Metaphors Using Cross-Linguistic Awareness-Raising Activities." English Language Teaching Journal 51: 352-360. DOI: 10.1093/elt/51.4.352

Evans, Vyvyan, and Melanie Green. 2006. Cognitive Linguistics: An Introduction. Mahwah, NJ: Lawrence Erlbaum.

Göpferich, Susanne. 2008. Translationsprozessforschung. Stand, Methoden, Perspektiven. Tübingen: Narr.

Jakobsen, Arnt Lykke, Kristian T. H. Jensen, and Inger M. Mees. 2007. "Comparing Modalities: Idioms as a Case in Point." In Interpreting Studies and Beyond. A Tribute to Miriam Shlesinger, ed. by Franz Pöchhacker, Arnt Lykke Jakobsen, and Inger M. Mees, 217-249. Copenhagen: Samfundslitteratur.

Kövecses, Zoltan. 2005. Metaphor in Culture: Universality and Variation. Cambridge: Cambridge University Press. DOI: 10.1017/CBO9780511614408

Kussmaul, Paul. 2000. Kreatives Übersetzen. Tübingen: Stauffenburg.

Kussmaul, Paul. 2003. "Der Professionalität auf der Spur." In Traducta Navis. Festschrift zum 60. Geburtstag von Christiane Nord, ed. by Britta Nord, and Peter A. Schmitt, 131-149. Tübingen: Stauffenburg.

Lakoff, George. 1993. “The Contemporary Theory of Metaphor.” In Metaphor and Thought, 2nd ed., ed. by Andrew Ortony, 202-251. Cambridge: Cambridge University Press.

DOI: $10.1017 / \mathrm{CBO} 9781139173865.013$

Lakoff, George, and Mark Johnson. 1980. Metaphors We Live By. Chicago: University of Chicago Press. DOI: 10.7208/chicago/9780226470993.001.0001

Martikainen, Kati. 1999. What Happens to Metaphorical Expressions Relating to 'Comprehension' in the Processes and Products of Translation? A Think-Aloud Protocol Study. A Pro Gradu thesis, Savonlinna School of Translation Studies. University of Savonlinna.

Mauranen, Anna, and Pekka Kujamäki (eds). 2004. Translation Universals. Do They Exist? Amsterdam: John Benjamins. DOI: 10.1075/btl.48

Olohan, Maeve. 2004. Introducing Corpora in Translation Studies. London: Routledge.

Schäffner, Christina. 2004. "Metaphor and Translation: Some Implications of a Cognitive Approach." Journal of Pragmatics 36: 1253-1269. DOI: 10.1016/j.pragma.2003.10.012

Shuttleworth, Mark. 2011. "Translational Behaviour at the Frontiers of Scientific Knowledge: A Multilingual Investigation into Popular Science Metaphor in Translation.” Translator 17 (2): 301-323. [Special Issue: Science in Translation.] DOI: 10.1080/13556509.2011.10799491 
Sjørup, Annette C. 2008. "Metaphor Comprehension in Translation: Methodological Issues in a Pilot Study." In Looking at Eyes. Eye-Tracking Studies of Reading and Translation Processing [Copenhagen Studies in Language, 36], ed. by Susanne Göpferich, Arnt Lykke Jakobsen, and Inger M. Mees, 53-77. Copenhagen: Samfundslitteratur.

Sjørup, Annette C. 2011. “Cognitive Effort in Metaphor Translation: An Eye-Tracking Study.” In Cognitive Explorations of Translation, ed. by Sharon O'Brien, 197-214. London: Continuum. Tirkkonen-Condit, Sonja. 2001. "Metaphors in Translation Processes and Products." Quaderns. Revista de traducció 6: 11-15.

Tirkkonen-Condit, Sonja. 2002. "Metaphoric Expressions in Translation Processes." Across Languages and Cultures 3 (1): 101-116. DOI: 10.1556/Acr.3.2002.1.8

van Dijk, Teun A. 1995. "Interdisciplinarity." Discourse and Society 6 (4): 459-460.

DOI: $10.1177 / 0957926595006004001$ 


\title{
Investigating the conceptual-procedural distinction in the translation process
}

\author{
A relevance-theoretic analysis of micro \\ and macro translation units*
}

\author{
Fabio Alves and José Luiz Gonçalves \\ Federal University of Minas Gerais, Brazil / Federal University \\ of Ouro Preto, Brazil
}

\begin{abstract}
This chapter draws on relevance theory (Sperber and Wilson 1986/1995) and its application to translation (Gutt 2000) to investigate processing effort in translation in relation to two different types of encodings, namely conceptual and procedural encodings (Blakemore 2002; Wilson 2011). Building on the experimental paradigm of data triangulation in translation process research (Alves 2003; Jakobsen 2005), it analyses the translation processes of eight professional translators when performing a direct and an inverse translation task. The analysis focuses on the number and types of encodings found in micro/macro translation units (Alves and Vale 2009; 2011). Results suggest that processing effort in translation is greater in instances of procedural than conceptual encodings.
\end{abstract}

Keywords: translation process research, relevance theory, conceptual and procedural encodings, micro/macro translation units, processing effort in translation

\section{Introduction}

Effort has been identified as an indicator of problem solving in tasks involving reading and writing (Scardamalia and Bereiter 1991). Inherent to those tasks are tactics employed by readers and writers, such as re-reading, refocusing, reviewing

\footnotetext{
* Research funded by CNPq, the Brazilian Research Council (grant 307072/2008-8), and FAPEMIG, the Research Agency of the State of Minas Gerais (grants SHA/PPM-00280-10 and ETC-00009-11).
} 
and rewriting, which impact on execution time and allow for correlations between effort and the degree of investment in finding a solution. However, other than time investment, it is not at all clear how we can measure effort of the kind required from translators to produce their renderings. Processing effort in translation has been investigated taking into account internal/external support (PACTE 2008) and time pressure (Jensen 2001). Additionally, translation units and patterns of cognitive segmentation (Dragsted 2005; Jakobsen 2005) have been correlated with effort. More recently, Alves and Vale $(2009 ; 2011)$ have shown that effort can be correlated with micro/macro units in the translation process through a corpus-linguistics oriented approach. Nevertheless, investigating processing effort in translation remains a challenge. Relevance theory (RT; Sperber and Wilson 1986/1995), which, in terms of inferential processing, postulates an optimal cognitive relation between the minimum processing effort necessary for the generation of the maximum cognitive effects possible, offers a productive way to investigate the role of effort in translation task resolution. The relevance-theoretic distinction between conceptual and procedural encodings (Blakemore 1987; 2002), we will argue, can be instrumental in analysing micro/macro translation units in target text production. Drawing on the RT framework, we assume that effort in translation is higher when processing deals with procedural rather than conceptual encodings. In this chapter, we seek to investigate if this claim can be assessed experimentally in translation process research (TPR henceforth). We posit that this assumption may be relevant for the description and explanation of the translation process itself. By investigating the allocation of processing effort in translation from a relevance-theoretic perspective, we aim to shed light on issues relevant to other areas, such as language and communication studies as well as expertise studies and cognitive science, thus contributing to the understanding of translation as an object of interdisciplinary inquiry.

\section{Theoretical underpinnings}

This section is divided into three subsections. First, we present a brief overview of the application of RT to translation as well as the defence of a cause-effect experimental paradigm to investigate translation processes. Next, we introduce some key relevance-theoretic concepts, including the distinction between conceptual and procedural encodings. Finally, we formulate the theoretical problem to be investigated experimentally in the remaining sections. 


\subsection{Relevance and translation: in search of a cause-effect relation}

Gutt (2000) states that Sperber and Wilson have posited a cognitive core which appears to be central to all human communication efforts and, therefore, makes human communication across language and cultural boundaries possible. Gutt argues that one of the most significant contributions of RT is to provide a causeeffect framework that is applicable to research in translation. Consequently, Gutt advocates in favour of what he calls CORT, namely, competence-oriented research of translation, aiming at understanding and explicating the mental faculties that enable human beings to translate.

For Gutt (2000), the works of Blakemore (1987) and Wilson and Sperber (1993), among others, have shown that elements of language can encode processing instructions which provide guidance to the audience as to how an expression is intended to be relevant. Gutt also points out that these instructions are empirically grounded and can be used to make testable predictions about the success and failure of human communication. In this chapter, we propose that the cause-effect framework provided by RT can be used to predict problems when the stimuli convey linguistic encodings which are needed for consistency with the principle of relevance.

\subsection{Some important relevance theory concepts}

\subsubsection{The principle of relevance and the effect-effort relation}

Sperber and Wilson (1986/1995) postulate a principle of relevance according to which, in any given inferential process, the human being's cognitive environment searches for the generation of the maximum cognitive effects possible while spending the minimum processing effort necessary to achieve this end. In RT, processing effort is the estimated amount of cognitive resources spent in any inferential process; while effects are the resulting changes observed in the person's cognitive environment after that inferential event, through which some mental representations are added, eliminated, reinforced or weakened. The principle of relevance establishes an optimal balance between the estimated effort to be spent and the effects to be obtained, always looking for a positive relation. Such effects, called contextual or cognitive effects in RT, correspond to the changes taking place in the cognitive environment as a result of inferential processes (i.e., the pieces of information added, changed or even excluded); effort, in turn, is the amount of cognitive resources spent in those processes. None of them can be measured in terms of precise amounts - they can only be estimated and treated in comparative dimensions. Therefore, for any input to be considered more or less relevant, it will depend on the balance between these two factors (effects and effort). An 
inferential process is to be considered relevant or more relevant, or presents a positive function in terms of relevance, if the contextual/cognitive effects outweigh the processing effort, and is to be considered less relevant or irrelevant, or presents a negative function, the other way round.

As regards the role of effort in the principle of relevance, Gutt (1998) argues that it could be withdrawn from the core of RT since it is not exactly the amount of effort that determines whether an inferential process will be assessed as more or less relevant, but the contextual/cognitive effects it generates. Therefore, Gutt defends the idea that the relevance of an utterance or other stimuli is proportional to its cognitive effects, irrespective of the amount of processing effort. Therefore, processing effort should be used only as a cognitive parameter to estimate if the processing event is worth being implemented.

However, Alves (2007) argues that, from an empirical-experimental perspective, it is worth preserving the initial postulation of the principle of relevance, as the relation between effort and effect is responsible for a positive (or negative) function in the cognitive system. Being relevant/more relevant or less relevant/irrelevant will depend on that positive or negative function, respectively. The experimental results shown in Alves (2007) seem to provide evidence in this direction. In this chapter, we build on Alves (2007) and propose that the effort/effect relation is paramount for determining the success of any cognitive event in translation.

\subsubsection{The conceptual-procedural distinction in relevance theory}

Blakemore (1987) introduced the conceptual-procedural distinction into RT to account for differences between regular content words and discourse connectives. In relevance-theoretic terms, conceptually encoded information is encoded by open lexical categories, such as nouns, adjectives and verbs, to convey conceptual meaning which is propositionally extendable and contributes to the inferential processing of an utterance. Procedurally encoded information, on the other hand, is encoded via non-open morphological categories, such as negation, tenses, determiners, word order, etc. It cannot be extended in propositional terms, but contributes decisively to the cognitive processing of an utterance by imposing procedural constraints on the construction of intended contexts and cognitive effects.

Wilson (2011) points out that initially the conceptual-procedural distinction coincided with the distinction between truth-conditional and non-truthconditional meaning. Regular content words were analysed as encoding concepts whereas discourse connectives contributed only to the derivation of implicatures. However, as Wilson further explains, the works of Wilson and Sperber (1993) and Blakemore (2002), among others, showed that the parallelism between the conceptual-procedural distinction and the truth-conditional vs. non-truth-conditional distinction breaks down in several ways. 
Wilson and Sperber's (1993) analysis of the illocutionary adverbial "seriously" in "Seriously, Bill is leaving" compared to the synonymous manner adverbial in "John spoke seriously to Anne" shows that both instances encode concepts. However, whereas the latter can be considered a regular content word that contributes to the truth conditions of the utterance, the former does not contribute to the truth-conditional content of "Seriously, Bill is leaving" but to its higher-order explicature ${ }^{1}$ by carrying information about the speaker's propositional or affective attitude. Wilson and Sperber (1993) argue that, since higher-order explicatures make no difference to truth-conditional content, "seriously" is both conceptual and non-truth conditional.

Wilson (2011) argues that another way in which that parallelism breaks down is when pronouns, such as 'I' and 'he', or indexicals, such as 'now' and 'then', depend on their referents, which vary from context to context and have to be pragmatically inferred. Wilson and Sperber (1993) suggest that such items should be analysed as encoding procedural constraints on the inferential phase of comprehension. However, unlike discourse connectives, they do contribute to the identification of the speaker's assertion and, therefore, contribute to the truth-conditional content of the utterance.

Additionally, Wilson and Sperber (1993) have shown that a vast array of nontruth-conditional items such as mood indicators, sentence and discourse particles, interjections and intonation encode a different type of procedural constraint on the construction of higher-order explicatures by carrying speech-act, propositional-attitude or affective-attitude information.

Wilson $(2011,9)$ argues that what distinguishes the conceptual-procedural distinction from the traditional semantic or pragmatic distinction is that "it carries definite cognitive commitments". For RT, to say that a certain expression encodes conceptual or procedural meaning is to say that it has implications for the nature of the cognitive mechanisms involved. Despite the fairly widespread view that the conceptual-procedural distinction is intended to be mutually exclusive, Wilson argues that there is little textual evidence to support this interpretation of relevance theory and advocates that conceptual and procedural meaning should not be treated that way.

1. On the distinction between explicatures and implicatures, Sperber and Wilson (1986/1995, 182) argue that "Any assumption communicated, but not explicitly so, is implicitly communicated: it is an implicature. [...] An explicature is a combination of linguistically encoded and contextually inferred conceptual features. The smaller the relative contribution of the contextual features, the more explicit the explicature will be, and inversely. Explicitness, so understood, is both classificatory and comparative: a communicated assumption is either an explicature or an implicature, but an explicature is explicit to a greater or lesser degree." 


\subsection{Revisiting the conceptual-procedural distinction in translation}

In RT terms, it follows that (1) the function of procedural expressions is to activate domain-specific cognitive procedures (i.e., morph-syntactic constraints in utterance processing) and (2) most content words also carry some procedural meaning. Therefore, processing effort in translation concentrates more on instances of procedural than conceptual encodings. Alves and Gonçalves (2003) and Alves (2007) have shown that there is a relation between processing effort and cognitive effect and also that the conceptual-procedural distinction plays a role in that process, but both were small-scale studies and only offer qualitative results. In this chapter, we attempt to tackle the conceptual-procedural distinction in a larger study, as described below.

\section{Methodological framework}

This section presents the experimental design, the methodology for data analysis including procedures for annotating translation process data, and the hypotheses to be tested.

\subsection{Experimental design}

The data investigated here was first analysed by Alves, Pagano and Silva (2009) in a study on directionality in translation. From their data, we selected eight translators with five or more years of professional experience in a wide range of domains, translating into both directions, and having translation as their main source of income. All subjects performed a direct and an inverse translation task under experimental conditions. Portuguese was the L1 of all subjects and English was their L2.

The two source texts were of a similar length (approximately 250 words), of the same text type (research article introductions published in scientific journals), from the same domain (medicine: sickle cell disease) and of similar rhetorical complexity as assessed on the basis of Rhetorical Structure Theory (Taboada and Mann 2006).

Task order was shifted randomly among subjects in order to control for any facilitating effect of domain knowledge. Subjects' performance was recorded using the key-logging software Translog in conjunction with the Tobii T60 eye tracker. However, only key-logged data are analysed in this chapter. All subjects were familiar with the QWERTY keyboard used for data collection.

Prior to each translation task, subjects performed a copy test in the same language they were translating into (114 words in Portuguese, for direct translation, and 110 words in English, for inverse translation) to serve as a baseline measurement of their typing skills. The copy tests were also recorded using Translog and Tobii T60. 


\subsection{Procedures for data analysis}

First, we annotated the two source texts to mark instances of conceptual and procedural encodings. Consistent with recent developments in RT (Wilson 2011), we also marked hybrid encoding categories when conceptual encodings also showed procedural meaning.

In order to establish that the source texts were comparable, we then checked for consistency between the number of encodings in the two source texts (see Appendix). Next, we annotated the Translog XML files using Litterae, an annotation and search system designed and implemented as a tool to store, annotate and query translation process data (for further details, see Alves and Vale 2009; 2011).

In this chapter, we follow Jakobsen (2005) and apply a 2.4-second pause interval to segment process data, since this parameter has been shown to be more consistent for the purpose of translation unit segmentation.

The procedure for annotating micro/macro TUs basically involves highlighting those units (lines) where related editing procedures take place during text production and grouping them together. In order to check which micro TUs are related to an initial TU, or the first production of a target text segment, the mouse pointer is passed over the lines following the first highlighted unit, observing if there is any kind of change in the text produced initially. Whenever a change occurs, the unit (line) on which the mouse pointer rests must be highlighted, thus integrating it into the macro TU (see Figure 1).

According to Alves and Vale $(2011,107)$, a micro TU is “ [...] defined as the flow of continuous TT production - which may incorporate the continuous reading of source and TT segments - separated by pauses during the translation process as registered by key-logging and/or eye-tracking software. It can be correlated to a ST segment that attracts the translator's focus of attention at a given moment." A macro TU, on the other hand, is "[...] defined as a collection of micro TUs that comprises all the interim text productions that follow the translator's focus on the same ST segment from the first tentative rendering to the final output that appears in the TT."

Alves and Vale (2011) classify macro TUs with editing procedures taking place only in the drafting phase as P1. Those macro TUs that are produced once in the drafting phase and changed only in the revision phase are classified as P2. Finally, those macro TUs that undergo editing procedures both during drafting and revision are classified as P3. In this chapter, we use Alves and Vale's (2011) classification. However, we broaden the taxonomy to include a P0 unit, corresponding to micro TUs that do not undergo any editing at all. For annotation purposes, P0 TUs are also considered macro TUs.

Since our subjects regularly translated into both directions and their performance had been assessed as comparable in direct and inverse translation (DT and 
IT, respectively) in Alves, Pagano and Silva (2009), we assume that there would be no significant differences in their segmentation patterns in DT and IT. Therefore, we assessed the subjects' performance using the taxonomy proposed by Alves and Vale (2011) expecting that similarities in DT and IT found by Alves, Pagano and Silva (2009) would also stand when other parameters were taken into account.

In our attempt to map instances of conceptual and procedural encodings onto translation process data, we have also annotated more detailed editing procedures inside each macro TU. We based our distinctions on two types of annotation parameters: (a) the level of linguistic complexity in an editing procedure; and (b) the distance between this change and the respective initial micro TU. For the analyses that follow, we assume that both parameters are related to processing effort. In other words, the higher the linguistic complexity involved in the editing procedure and the farther it is from the respective initial micro TU, the greater the processing effort required.

For parameter (a), we introduced the following categories: $[t]$ typos; $[c]$ conclusion of a lexical item out of the linear production, generally in another micro $\mathrm{TU}$; [1] editing of lexical item; [m] editing of a morph-syntactic element; and [p] editing of complex phrasal structures. As regards parameter (b), we introduced four categories marked by asterisks: $\left(^{*}\right)$ - changes inside the initial micro TU; $\left({ }^{*}\right)$ - changes in a micro TU following and very close to the initial micro TU; $\left({ }^{* * *}\right)$ - changes in a farther subsequent micro TU, still in the drafting phase; and $\left({ }^{* * *}\right)$ - changes in the end-revision phase. For both parameters, the order of the items assumes a progressive level of complexity.

\subsection{Hypotheses}

In light of the theoretical considerations presented in Section 1 and the methodological framework detailed in the previous subsections, we are now in a position to formulate two working hypotheses to be tested on the translation process data annotated in Litterae.

(1) Given that, in RT terms, instances of procedural encodings (PE) are more prevalent than cases of conceptual encodings (CE), processing effort within macro TUs will be higher in instances directly related to PE than to CE.

This does not necessarily follow from the number of encodings mapped onto the source texts but is rather related to the pauses and regressions identified in a macro $\mathrm{TU}$ which, we assume, are considered to be indicators of effortful target text production. 
(2) Given that, in RT terms, instances of procedural encodings (PE) are more prevalent than cases of conceptual encodings (CE), processing effort within macro TUs, as assessed by distance indicators, will be greater for instances of $\mathrm{PE}$ than for cases of CE.

In short, the higher the linguistic complexity involved in the editing procedure and the farther it is from the respective initial micro TU, the greater the processing effort required. By including a spatial dimension in the analysis of processing effort in translation, we introduce a new component to the modeling of effort that generally uses the notion of time spent on the task to assess (expert) performance. The next section will present the data analysis and a discussion on relevance-theoretic aspects related to the conceptual-procedural distinction and their impact on processing effort in translation.

\section{Analyses and discussion}

In this section, we present the results of our study and analyse them from a relevance-theoretic perspective applied to TPR. First, we analyse the number and type of macro TUs produced in direct (DT) and inverse translation (IT) tasks and compare our results with Alves and Vale's (2011). Next, we present an analysis of the editing annotations and relate them to the relevance-theoretic concepts of conceptual (CE) and procedural (PE) encodings, taking into account a hybrid category that entails both encodings. Finally, we assess the conceptual-procedural distinction on the basis of the effort undertaken by the subjects using distance indicators for micro TUs occurring within the same macro TU. We close the section with a discussion about the role of effort in relation to the conceptual-procedural distinction.

\subsection{Types of macro translation units in direct/inverse translation tasks}

Considering that our data are numerical and deal with paired samples, we decided to apply paired T-tests to validate statistically some of the differences found between pairs of mean values, adopting a $95 \%$ confidence level to consider the differences statistically significant. Table 1 shows the number of macro TUs per subject and translation direction.

Results show that there were no significant differences regarding directionality (DT/IT), which reinforces comparability of results in the two tasks. When comparing overall mean values between pairs of macro TU categories for both DT/IT, P0 is significantly higher than P2 and P3. In addition, P1 is significantly 
Table 1. Macro TUs per subject in DT/IT (absolute and mean values)

\begin{tabular}{|c|c|c|c|c|c|c|c|c|c|c|c|c|c|c|c|c|c|c|c|}
\hline \multirow{2}{*}{$\begin{array}{r}\text { Subject } \\
\text { Macro TUs }\end{array}$} & \multicolumn{2}{|c|}{ Jane } & \multicolumn{2}{|c|}{ Cycy } & \multicolumn{2}{|c|}{ Adam } & \multicolumn{2}{|c|}{ Jim } & \multicolumn{2}{|c|}{ Will } & \multicolumn{2}{|c|}{ Mona } & \multicolumn{2}{|c|}{ Tess } & \multicolumn{2}{|c|}{ Rui } & \multicolumn{2}{|c|}{ Means } & \multirow{2}{*}{$\begin{array}{r}\text { Overall } \\
\text { means }\end{array}$} \\
\hline & DT & IT & DT & IT & DT & IT & DT & IT & DT & IT & DT & IT & DT & IT & DT & IT & $D T$ & $I T$ & \\
\hline P0 & 64 & 79 & 59 & 41 & 18 & 16 & 22 & 8 & 39 & 27 & 5 & 2 & 18 & 12 & 48 & 46 & 4.13 & 28.88 & 31.50 \\
\hline P1 & 30 & 37 & 32 & 30 & 40 & 21 & 34 & 24 & 19 & 10 & 7 & 5 & 24 & 18 & 24 & 29 & 26.25 & 21.75 & 24.00 \\
\hline P2 & 0 & 4 & 0 & 0 & 2 & 0 & 0 & 2 & 11 & 17 & 0 & 2 & 4 & 2 & 1 & 2 & 2.25 & 3.63 & 2.94 \\
\hline P3 & 2 & 6 & 1 & 3 & 2 & 3 & 6 & 3 & 10 & 19 & 16 & 15 & 17 & 10 & 3 & 1 & 7.13 & 7.50 & 7.31 \\
\hline Total & 96 & 126 & 92 & 74 & 62 & 40 & 62 & 37 & 79 & 73 & 28 & 24 & 63 & 42 & 76 & 78 & 69.75 & 61.75 & 65.75 \\
\hline
\end{tabular}

(Paired T-tests for the overall means $[\mathrm{n}=16 ; \mathrm{DF}=15 ; \alpha=0.05]-\mathrm{P} 0$ vs $\mathrm{P} 1$ : $\mathrm{p}=0.14$ [non-significant]; $\mathrm{P} 0$ vs $\mathrm{P} 2$ : $\mathrm{p}<0.01$; P0 vs P3: $\mathrm{p}<0.01 ; \mathrm{P} 1$ vs $\mathrm{P} 2$ : $\mathrm{p}<0.01 ; \mathrm{P} 1$ vs $\mathrm{P} 3$ : $\mathrm{p}<0.01 ; \mathrm{P} 2$ vs $\mathrm{P} 3: \mathrm{p}<0.01 ;[\mathrm{P} 0+\mathrm{P} 1]$ vs $[\mathrm{P} 2+\mathrm{P} 3]$ : $\mathrm{p}<0.01)$

higher than P2 and P3. These trends are consistent with the claim that P0 and P1 are different from $\mathrm{P} 2$ and $\mathrm{P} 3$ in terms of processing effort.

The findings for $\mathrm{P} 1$, and indirectly for $\mathrm{P} 0$, confirm the results of Alves and Vale (2011), who focused on different text types and different language combinations to analyse the performance of another group of subjects. Thus, the results in Table 1 suggest that routinised (proceduralised) behaviour is characteristic of P0. To a lesser degree, routinised behaviour also applies to P1. Both P0 and P1 play a crucial role in the translation process in terms of occurrences. When performance is compared intrasubjectively, there is a clear tendency of $\mathrm{P} 0$ and $\mathrm{P} 1$ being far more frequent than P2 and P3. This seems to indicate two different patterns of cognitive processing among subjects: one pattern favouring an immediate rendering (P0/ $\mathrm{P} 1)$ and another pattern of a more recursive nature (P2/P3).

In terms of the allocation of processing effort, we assume that there is a progression from $\mathrm{P} 0$ to $\mathrm{P} 3$. Cognitive processing effort is considered to be somewhat smaller for P0 TUs as their implementation takes place in the cognitive flow, with no interruptions that could signal problem-solving activity. Thus, we may argue that P0 TUs occur in the so-called Adhoc Block (see Königs 1987; Alves 1995) and point to automatic, routinised (proceduralised) processes. P1 also shows similar automatic processes to P0 but entails some level of metacognitive monitoring. P2 and P3 macro TUs, on the other hand, involve progressively higher degrees of metacognitive activity taking place in the so-called Rest Block (Königs 1987; Alves 1995 ) and point to more conscious and reflective cognitive activity related to problem-solving and decision-making processes. Drawing on Elman et al. (1996), we assume that the more activated a piece of information in working memory is, the less effort is required to access and process it. In terms of the theoretical and methodological frameworks proposed in this work, P1 TUs include editing procedures closer to the cognitive flow for the initial production of that unit; P2 units include procedures distant in time from the initial production, requiring extra effort to reactivate those pieces of information; and $\mathrm{P} 3$ units include both closer (in the drafting phase) and distant (in the revision phase) editing procedures, which may be assessed as more effort consuming than the two previous categories. 
Taking the relevance-theoretic framework into account, we could argue that in translation, as in any other cognitive task, the human cognitive system looks for economy. Therefore, it tries to use a routinised form of processing or, at least, keep processing as close as possible to routinised behaviour. However, we know that translation involves a considerable amount of problem-solving and decisionmaking processes that take place at the higher levels of the human cognitive system and consume considerably more processing effort (e.g., Shreve 2006). Thus, whenever a translation task increases in complexity, there seems to be a tendency to move away from routinised behaviour to engage in more conscious processing effort in order to solve translation problems.

\subsection{Editing procedures in micro/macro translation units}

Figure 1 shows an example of annotations in a P1 macro TU in terms of processing characteristics with the respective tagging of the categories of editing procedures, $[\mathrm{t}],[\mathrm{c}],[1],[\mathrm{m}]$ and $[\mathrm{p}]$, related to different levels of linguistic complexity, and the distance indicators $\left({ }^{*}\right),\left(^{* *}\right),\left(^{* * *}\right)$ and $\left({ }^{* * *}\right)$.

The initial micro TU accounts for the production of are. The two following lines constitute the initial processing block (two-asterisk changes: ${ }^{* *}$ ). In terms of editing procedures, we tagged the replacement of are by form as a change related to lexical choice [1] and, therefore, as $\mathrm{P}^{\star *} 1$. In light of the latest relevance-theoretic discussions about the conceptual-procedural distinction, we are aware that there is also some kind of procedural adjustment in the replacement of are by form. However, we decided to follow a more traditional relevance-theoretic account to deal with the encoding of individual items (see Blakemore 2002). Thus, items are tagged according to their most prominent encoding function — in this case [1].

However, in a situation where both conceptual and procedural encodings are processed in a larger chunk, we have tagged it as [p] (i.e., a hybrid category encompassing both conceptual and procedural meaning; see Wilson 2011). This can be seen, for instance, when qualitative hemoglobine is inserted before the noun ailments. Besides being a lexical insertion [1], it also changes the structure of the noun phrase and works as a procedural constraint on the processing of ailments. Therefore, this insertion encompasses changes in both conceptual and procedural meaning and was tagged as $[\mathrm{p}]$ in $\mathrm{P} 1^{\star *} \mathrm{p}$.

\begin{tabular}{|c|c|c|c|c|c|c|}
\hline Apply tag & Tag Name & $P 1^{\star \star-} \mid$ lu2 & $P 1^{\text {*- }} p$ lu2 & $\mathrm{P} 1^{\mathrm{x}-\mathrm{x}-\mathrm{x}} \mathrm{t}$ lu2 & $\mathrm{P} 1^{\text {k**x} \mid \text { lu2 }} \mathrm{P} 1$ lu2 & Delete \\
\hline \multirow{5}{*}{\multicolumn{2}{|c|}{$\begin{array}{c}\text { are }\left.\right|^{\star *} \mid \text { a set of ailments }\left.\right|^{\star *} p \text { a } \\
\text { set of qualitative hemoglobine } \\
\text { ailments }\left.\right|^{\star \star *} \text { hemoglobin }\left.\right|^{\star \star *} \mid \\
\text { diseases }\end{array}$}} & \multicolumn{5}{|c|}{ 384179: are } \\
\hline & & \multicolumn{5}{|c|}{ 387917: $\otimes \otimes \otimes \otimes$ form a set of ailments } \\
\hline & & \multicolumn{5}{|c|}{ 398719: $\leftarrow \leftarrow \leftarrow \leftarrow \leftarrow \leftarrow \leftarrow \leftarrow \leftarrow$ qualitative hemoglobine $\rightarrow \rightarrow \rightarrow \rightarrow \rightarrow \rightarrow \rightarrow \rightarrow$} \\
\hline & & \multicolumn{5}{|c|}{ 514908: [Mouse][Mouse, 1]区[Mouse][Mouse, 1] $\bowtie$} \\
\hline & & \multicolumn{5}{|c|}{ 940616: [Mouse][Mouse, 8]diseases } \\
\hline
\end{tabular}

Figure 1. Macro TU with its micro-procedure tagging through Litterae [Rui-IT] 
The fourth line, tagged $\mathrm{P} 1^{\star * *} t$, represents a micro TU outside the initial processing block where a typing mistake is corrected: the final $e$ in hemoglobine is deleted. Finally, the word ailments was replaced by diseases and was tagged as [1] in what constitutes a $\mathrm{P}^{\star * *} 1$ micro TU. Had there been a further lexical change in the end-revision phase, it would have been tagged as a four-asterisk change $\left({ }^{* * *} 1\right)$.

Table 2 shows the number and type of editing procedures within each micro TU for each subject and translation direction.

In view of the large number of [ $t$ ] annotations, one could hypothesise that they occur so frequently not primarily because of the subjects' lack of typing skills, but mostly due to the degree of cognitive overload related to the type of complex parallel processing that takes place during translation. However, there is no significant difference in the number of typos [ $\mathrm{t}$ ] in the copy tests and in the translation tasks. As the number of typos is equally high for all subjects and both translation directions, it is assumed that this data category has no relevance for the present analysis.

As shown in Table 2, the least frequent category was [c], the conclusion of a lexical item in an immediately subsequent micro TU. At first glance, [c] could be considered a particular type of typing problem. However, one could assume that the interruption in the production flow of the lexical item is most probably related to parallel processing activities competing for the allocation of cognitive resources. In other words, whenever demand for processing effort in translation exceeds a certain threshold, typing, and most likely the cognitive flow, is interrupted. As a consequence, the micro TU breaks down. Since there is no editing of a semantic nature (i.e., no problem regarding lexical choice) we differentiate [c] from [1].

Having accounted for the occurrences of $[t]$ and $[c]$, we are now left with occurrences of [1], [m], and [p], which are closely related to the relevance-theoretic concepts of conceptual and procedural encodings. The total number of conceptual encodings (CE) is considered to be equivalent to the sum of $\{[1]+[\mathrm{p}]\}$, and the total number of procedural encodings (PE) equal to the sum of $\{[\mathrm{m}]+[\mathrm{p}]\}$.

Table 2. Editing procedures per subject in DT/IT (absolute and mean values)

\begin{tabular}{|c|c|c|c|c|c|c|c|c|c|c|c|c|c|c|c|c|c|c|c|}
\hline \multirow{2}{*}{$\begin{array}{r}\text { Subject } \\
\text { Macro TUs }\end{array}$} & \multicolumn{2}{|c|}{ Jane } & \multicolumn{2}{|c|}{ Cycy } & \multicolumn{2}{|c|}{ Adam } & \multicolumn{2}{|c|}{ Jim } & \multicolumn{2}{|c|}{ Will } & \multicolumn{2}{|c|}{ Mona } & \multicolumn{2}{|c|}{ Tess } & \multicolumn{2}{|c|}{ Rui } & \multicolumn{2}{|c|}{ Means } & \multirow{2}{*}{$\begin{array}{r}\text { Overall } \\
\text { means }\end{array}$} \\
\hline & DT & IT & DT & IT & DT & IT & DT & IT & DT & IT & DT & IT & DT & IT & DT & IT & $D T$ & $I T$ & \\
\hline$t$ & 28 & 33 & 36 & 32 & 58 & 35 & 77 & 62 & 25 & 37 & 53 & 76 & 85 & 72 & 15 & 18 & 47.13 & 45.63 & 46.38 \\
\hline c & & 9 & 2 & 4 & 1 & 3 & 7 & 11 & 14 & 13 & 2 & 1 & 3 & 8 & 3 & 0 & 5.00 & 6.88 & .94 \\
\hline 1 & 7 & 15 & 6 & 8 & 18 & 10 & 16 & 16 & 19 & 36 & 11 & 5 & 13 & 13 & 4 & 8 & 11.75 & 13.88 & 12.81 \\
\hline m & 11 & 19 & 20 & 13 & 20 & 16 & 17 & 7 & 20 & 35 & 12 & 17 & 28 & 14 & 13 & 14 & 17.63 & 16.88 & 17.25 \\
\hline $\mathbf{p}$ & 12 & 18 & 2 & 1 & 4 & 13 & 2 & 4 & 0 & 4 & 7 & 8 & 6 & 4 & 7 & 14 & 5.00 & 8.25 & 6.63 \\
\hline Total & 64 & 94 & 66 & 58 & 101 & 77 & 119 & 100 & 78 & 125 & 85 & 107 & 137 & 111 & 42 & 60 & 86.50 & 91.50 & 89.00 \\
\hline
\end{tabular}

Notes: $\mathrm{t}=$ typos; $\mathrm{c}=$ concluding lexical item in another unit; $1=$ editing lexical item; $\mathrm{m}=$ editing morph-syntactic element; $\mathrm{p}=$ editing complex phrasal structures. $\mathrm{CE}=1+\mathrm{p}$ and $\mathrm{PE}=\mathrm{m}+\mathrm{p}$.

(Paired T-tests for the overall means $[n=16 ; D F=15 ; \alpha=0.05]-C E$ vs PE: $p=0.01$ ) 


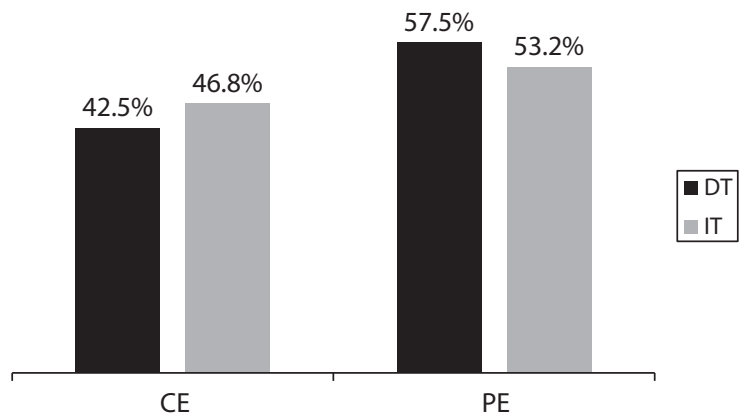

Figure 2. Number of editing procedures in relation to type of encoding (CE/PE) in DT/ IT (relative values)

We adopted this criterion since each [p] editing procedure includes, on average, one $[1]$ and one $[\mathrm{m}]$ occurrence.

With the exception of two subjects and only with IT, the number of editing procedures related to PE is higher than the values for CE (see Figure 2). As shown in Table 2, the difference between PE and CE for DT and IT combined is statistically significant (t-test, $p=0.01$ ).

The latest findings in RT point to a prevalent role of procedural meaning in the processing of utterances (Wilson 2011). The difference in the number of editing procedures related to $\mathrm{CE}$ and $\mathrm{PE}$ not only corroborates the relevance-theoretic assumption that PEs are also included in lexical items, formerly considered as exclusively conceptual, but it also validates them experimentally. Hypothesis 1 is therefore confirmed.

\subsection{Distance indicators of conceptual and procedural encodings}

In line with the relevance-theoretic predictions, our results show that subjects appear to concentrate their editing procedures during the cognitive flow of text production or as close to it as possible in order to maximise cognitive efficiency (see Table 3).

Table 3. Number of editing procedures in relation to distance from initial micro TU (absolute and mean values)

\begin{tabular}{|c|c|c|c|c|c|c|c|c|c|c|c|c|c|c|c|c|c|c|c|}
\hline \multirow{2}{*}{$\begin{array}{r}\text { Subject } \\
\text { Macro TUs }\end{array}$} & \multicolumn{2}{|c|}{ Jane } & \multicolumn{2}{|c|}{ Cycy } & \multicolumn{2}{|c|}{ Adam } & \multicolumn{2}{|c|}{ Jim } & \multicolumn{2}{|c|}{ Will } & \multicolumn{2}{|c|}{ Mona } & \multicolumn{2}{|c|}{ Tess } & \multicolumn{2}{|c|}{ Rui } & \multicolumn{2}{|c|}{ Means } & \multirow{2}{*}{$\begin{array}{r}\text { Overall } \\
\text { means }\end{array}$} \\
\hline & DT & IT & DT & IT & DT & IT & DT & IT & DT & IT & DT & IT & DT & IT & DT & IT & $D T$ & $I T$ & \\
\hline$\%$ & 19 & 24 & 35 & 35 & 67 & 43 & 86 & 64 & 22 & 28 & 58 & 73 & 79 & 61 & 14 & 12 & 47.50 & 42.50 & 45.00 \\
\hline$* *$ & 32 & 53 & 20 & 20 & 20 & 27 & 11 & 23 & 24 & 22 & 5 & 2 & 17 & 16 & 16 & 36 & 18.13 & 24.88 & 21.50 \\
\hline$* * *$ & 11 & 6 & 9 & 0 & 8 & 3 & 9 & 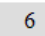 & 2 & 0 & 1 & 1 & 5 & 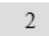 & 7 & 7 & 6.50 & 3.125 & 4.81 \\
\hline 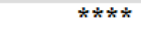 & 2 & 11 & 2 & 3 & 6 & 4 & 13 & 7 & 30 & 75 & 21 & 31 & 36 & 32 & 5 & 5 & 14.38 & 21.00 & 17.69 \\
\hline Total & 64 & 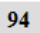 & 66 & 58 & 101 & 77 & 119 & 100 & 78 & 125 & 85 & 107 & 137 & 111 & 42 & 60 & 86.50 & 91.50 & 89.00 \\
\hline
\end{tabular}

(Paired T-tests for the overall means [n=16; DF=15; $\alpha=0.05]-{ }^{*} \mathrm{vs} * *: \mathrm{p}<0.01 ; * \mathrm{vs} * * *: \mathrm{p}<0.01 ; * \mathrm{vs} * * * * ; \mathrm{p}<0.01$; ** vs***: $\mathrm{p}<0.01 ; * *$ vs ****: $\mathrm{p}=0.28$ [non-significant]; *** vs ****: $\mathrm{p}=0.016$ ) 
The one-asterisk changes $\left({ }^{*}\right)$, located within the cognitive flow of text production, are significantly more frequent than any of the others (T-tests, $p<0.05$ ). These are followed by two-asterisk changes ${ }^{* *}$, located immediately after the cognitive flow of text production in the subsequent micro TUs (the initial block of a macro TU), which are significantly more frequent than $\left(^{\star * *}\right)$ changes. The high frequency of $\left(^{*}\right)$ and $\left({ }^{*}\right)$ changes relate to the prevalence of P0 and P1 shown in Table 1 and provide evidence that processing effort in translation, as shown by editing procedures, is mostly allocated during the flow of text production or in its immediate vicinity. The next most frequent are four-asterisk changes $\left(^{\star * * *}\right)$, located in the end-revision phase. Finally, three-asterisk changes $\left({ }^{* *}\right)$, located at a remote distance within the drafting phase, are the least frequent. There are no significant differences between $\left({ }^{* * *}\right)$ and $\left({ }^{* *}\right)$ changes.

These results suggest that the allocation of cognitive resources in translation progresses from $\left({ }^{*}\right)\left(^{* *}\right)>\left({ }^{* * *}\right)>\left({ }^{* * *}\right)$ or $\mathrm{P} 0>\mathrm{P} 1>\mathrm{P} 3>\mathrm{P} 2$ (as shown in Table 1). Drawing on relevance-theoretic assumptions, we could argue that subjects concentrate editing procedures within or very close to the respective initial micro $\mathrm{TU}$ and systematically attempt to reduce cognitive processing effort in order to optimize the resources in their cognitive environments. If they postpone the solution to a problem, or only fully realize this problem later on, the required processing effort needed to re-activate relevant information will be counter-productive in terms of cognitive processing economy. This is consistent with the relevancetheoretic framework, since additional processing effort diminishes the relevance of the cognitive effects.

\subsection{Processing effort in relation to conceptual and procedural encodings}

The number of editing procedures related to $\mathrm{CE}$ and PE (i.e. excluding [t] and [c]) for the two translation directions are shown in Figure 3 with respect to the relative distance indicators. Although most differences in the distribution are not statistically significant, a qualitative analysis of the data allows for some relevant observations.

The total number of occurrences for $\mathrm{CE}$ and $\mathrm{PE}$ is highest in $\left(^{* *}\right)$, followed by $(* * *)$. We assume that this can be interpreted in terms of allocation of effort to phases in the translation process, indicating where effort is greater. In $\left(^{* *}\right)$, subjects interrupt the cognitive flow to deal with more immediate processing problems. In $\left(^{* * *}\right)$, however, problem solving is postponed to the end-revision phase. For both $\left(^{* *}\right)$ and $\left({ }^{* * *}\right)$, PE is greater than CE.

We are aware that the patterns differ slightly if we consider DT and IT separately. We would argue, however, that the distribution of encodings in source texts and the analysis of macro TUs and types of encoding in the previous subsections 


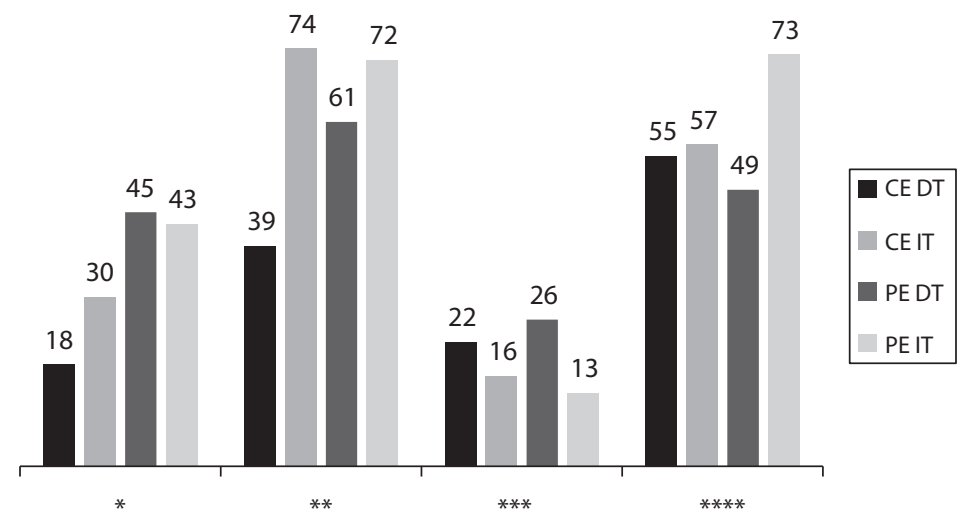

Figure 3. Distance indicators with respect to conceptual and procedural encodings (absolute values)

show that there is a balanced distribution in the data. Therefore, the analysis of DT/IT in terms of distance indicators and types of encoding suggest that there are more instances of PE than CE in the translation process. It also shows that these instances predominantly occur immediately after a micro TU is taken up and later on in the process at the end-revision phase where more reflective forms of problem solving and decision making probably take place (Alves 2007).

Although not all the results are statistically significant, data analysis shows the prevalence of processing effort for PE in absolute terms and points to the predominant role of PE in translation, particularly in $\left(^{(*}\right)$ and $\left({ }^{* * *}\right)$, where processing effort seems to be concentrated. Although Hypothesis 2 is not confirmed statistically, it is congruent with the overall results in absolute numbers. It remains to be seen if research involving a larger number of subjects would confirm this tendency and validate it statistically, thus experimentally corroborating relevance-theoretic assumptions.

\section{Concluding remarks}

Our results point to productive distinctions in the cognitive processing of conceptual (CE) and procedural (PE) encodings in translation. Our working hypotheses were partially confirmed: (1) overall, there are significantly more instances of PE than cases of CE in macro TUs; (2) processing effort as measured by distance indicators in translation seems greater for instances related to PE than for cases of $\mathrm{CE}$, particularly in $\left(^{* *}\right)$ and $\left({ }^{* * *}\right)$. Not all results concerning distance indicators were statistically significant. However, there is evidence that not only points to the relevance-theoretic claims about the conceptual-procedural distinction, but also 
confirms that, in addition to the time spent on a task, the allocation of processing effort in translation can be spatially modelled (i.e., the farther micro TUs are located from the initial micro TU in the same macro TU, the greater the processing effort). To make more robust claims, other experimental studies should be carried out under the same experimental conditions in order to see if similar tendencies arise. Eye tracking could also be used to analyse effort in terms of number of eye fixations and/or fixation duration in areas of interest (AOIs) related to instances of conceptual and procedural encodings to see where the allocation of effort is greater. The data collected in an experiment integrating eye-tracking measurements and recordings of a second set of translation tasks performed by the same subjects is expected to provide answers to some of the questions that have arisen in our study.

The results discussed in this chapter clearly support a relevance-theoretic account of processing effort in translation. We have also shown that the conceptualprocedural distinction (Wilson 2011) offers an insightful and productive way to tap into translation process data. From an interdisciplinary perspective, the work has contributed to the study of processing effort in general, which may be relevant to such areas as expertise studies and cognitive science, and to the exploration of some recent developments in RT with respect to the analysis of translation. It has also contributed to validating some of RT's theoretical claims by means of an empirical-experimental approach.

\section{References}

Alves, Fabio. 1995. Zwischen Schweigen und Sprechen: Wie bildet sich eine transkulturelle Brücke? Hamburg: Dr. Kovac.

Alves, Fabio (ed). 2003. Triangulating Translation: Perspectives in Process Oriented Research

[Benjamins Translation Library, 41]. Amsterdam: John Benjamins. DOI: 10.1075/btl.45

Alves, Fabio. 2007. "Cognitive Effort and Contextual Effect in Translation: A RelevanceTheoretic Approach." Journal of Translation Studies 10 (1): 18-35.

Alves, Fabio, and José Luiz Gonçalves. 2003. "A Relevance Theory Approach to the Investigation of Inferential Processes in Translation.” In Triangulating Translation: Perspectives in Process Oriented Research, ed. by Fabio Alves, 3-24. Amsterdam: John Benjamins.

DOI: 10.1075/btl.45.04alv

Alves, Fabio, Adriana Pagano, and Igor Silva. 2009. "A New Window on Translators' Cognitive Activity: Methodological Issues in the Combined Use of Eye Tracking, Key Logging and Retrospective Protocols." In Methodology, Technology and Innovation in Translation Process Research. A Tribute to Arnt Lykke Jakobsen, ed. by Inger Mees, Fabio Alves, and Susanne Göpferich, 267-291. Copenhagen: Samfundslitteratur.

Alves, Fabio, and Daniel Vale. 2009. "Probing the Unit of Translation in Time: Aspects of the Design and Development of a Web Application for Storing, Annotating, and Querying 
Translation Process Data." Across Languages and Cultures 10 (2): 251-273.

DOI: 10.1556/Acr.10.2009.2.5

Alves, Fabio, and Daniel Vale. 2011. "On Drafting and Revision in Translation: A Corpus Linguistics Oriented Analysis of Translation Process Data." TC3. Translation: Corpora, Computation and Cognition 1 (1): 105-122.

Blakemore, Diane. 1987. Semantic Constraints on Relevance. Oxford: Blackwell.

Blakemore, Diane. 2002. Relevance and Linguistic Meaning: The Semantics and Pragmatics of Discourse Markers. Cambridge: Cambridge University Press.

DOI: 10.1017/CBO9780511486456

Dragsted, Barbara. 2005. "Segmentation in Translation. Differences Across Levels of Expertise and Difficulty." Target 17 (1): 49-70. DOI: 10.1075/target.17.1.04dra

Elman, Jeffrey L., Elizabeth A. Bates, Mark H. Johnson, Annette Karmiloff-Smith, Domenico Parisi, and Kim Plunkett. 1996. Rethinking Innateness: A Connectionist Perspective on Development. Cambridge, MA: MIT Press.

Gutt, Ernst-August. 1998. "Relevance and Effort: A Paper for Discussion.” Paper presented at the II Relevance Theory Workshop, 8-10 September 1998, University of Luton.

Gutt, Ernst-August. 2000. Translation and Relevance: Cognition and Context, 2nd ed. Manchester: St Jerome.

Jakobsen, Arnt. 2005. “Investigating Expert Translators' Processing Knowledge.” In Knowledge Systems and Translation, ed. by Helle V. Dam, Jan Engberg, and Heidrun GerzymischArbogast, 173-189. Berlin: Mouton de Gruyter.

Jensen, Astrid. 2001. The Effects of Time on Cognitive Processes and Strategies in Translation. Copenhagen: Working Papers in LSP.

Königs, Frank. 1987. "Was beim Übersetzen passiert: theoretische Aspekte, empirische Befunde und praktische Konsequenzen." Die neueren Sprachen 2: 162-185.

PACTE. 2008. "First Results of a Translation Competence Experiment: 'Knowledge of Translation' and 'Efficacy of the Translation Process.' In Translator and Interpreter Training. Issues, Methods and Debates, ed. by John Kearns, 104-126. London: Continuum.

Scardamalia, Marlene, and Carl Bereiter. 1991. "Literate Expertise." In Toward a General Theory of Expertise, ed. by Karl-Anders Ericsson, and John Smith, 172-194. Cambridge: Cambridge University Press.

Shreve, Gregory. 2006. “The Deliberate Practice: Translation and Expertise." Journal of Translation Studies 9 (1): 27-42.

Sperber, Dan, and Deirdre Wilson. 1986/1995. Relevance: Communication and Cognition, 2nd ed. Oxford: Blackwell.

Taboada, Maite, and William C. Mann. 2006. "Rhetorical Structure Theory: Looking Back and Moving Ahead.” Discourse Studies 8 (3): 423-459. DOI: 10.1177/1461445606061881

Wilson, Deirdre, and Dan Sperber. 1993. "Linguistic Form and Relevance." Lingua 90: 1-25. DOI: 10.1016/0024-3841(93)90058-5

Wilson, Deirdre. 2011. “The Conceptual-Procedural Distinction: Past, Present, and Future.” In Procedural Meaning: Problems and Perspectives, ed. by Victoria Escandell-Vidal, Manuel Leonetti, and Aoife Ahern, 3-28. London: Emerald Group.

DOI: $10.1108 /$ S1472-7870(2011)0000025005 


\title{
Appendix. Mapping conceptual and procedural encodings in the source texts
}

\author{
[Text 1 - for Direct Translation Task]
}

Coagulation activation and inflammation in sickle cell disease-associated pulmonary hypertension

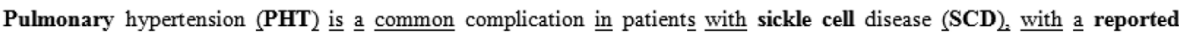
prevalence of approximately 30\%. Multiple studies have shown that PHT is associated with increased mortality in SCD patients. The pathogenesis of PHT in SCD is probably due to a variety of factors. Recent evidence suggests a central role for chronic intravascular hemolysis ${ }_{2}$ with associated scavenging of nitric oxide by cell-free plasma hemoglobin. Arginase, which converts $\mathrm{L}$-arginine (the substrate for nitric oxide synthesis) to omithine $\mathrm{e}_{\mathrm{a}}$ is also released following hemolysis. Elevated arginase activity ${ }_{2}$ and the resultant decrease in the arginine/ornithine ratio ${ }_{2}$ is associated with $\mathrm{PHT}_{\underline{1}} \mathrm{SCD}_{2}$

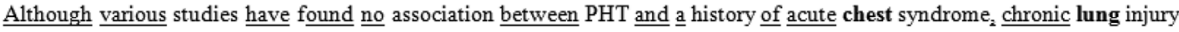
resulting from repeated episodes of acute chest syndrome may lead to the development of PHT due to chronic fibrotic pulmonary parenchymal damage $e_{z}$ altered vascular tone $e_{2}$ vascular proliferation hypoxia and consequent pulmonary vasculopathy. Finally, pulmonary thromboembolism and progressive endothelial damage with concentric pulmonary vascular intimal hyperplasia and in situ thrombosis may also contribute to the pathogenesis of PHT in SCD. The aim of the present study was to determine whether coagulation activation and inflammation are associated with PHT in SCD. Furthermore, we aimed to assess correlations between measures of coagulation activation and inflammation with markers of hemolysis. To address these questions, we evaluate $\underline{\text { ta }} \underline{\text { cohort }}$ of patients followed at an adult Sickle Cell Clinic.

Regular font $=$ conceptual encoding (89 units)

Underlined = procedural encoding (180 units)

Bold = conceptual encoding working as procedural encoding (51 units)

(245 words / Type-token ratio $=0.55$ )

\section{[Text 2 - for Inverse Translation Task]}

Hidroxiuréia em pacientes com síndromes falciformes acompanhados no $\underline{\text { no }}$ Hospital Hemope Recife_PE

Introdução

$\underline{\text { As }}$ síndromes falciformes (SF) constituem um conjunto de moléstias qualitativas da hemoglobina, nas quais herda-se o gene

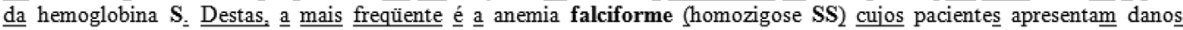
orgânicos desde a infância, resultantes dos episódios vaso-oclusivos repetidos. Vários estudos em adultos vêm demonstrando a eficácia do uso da hidroxiuréia ${ }^{\mathbf{H U}} 2$ cujo efeito principal é a elevação dos níveis de hemoglobina fetal $(\mathbf{H b F})$ em pacientes portadores de SF com pouco ou nenhum efeito colateral $\underline{\text { A HU }}$ é um agente quimioterápico bastante conhecido e utilizado para tratamento de síndromes mieloproliferativas como leucemia mielóide crônica e policitemia vera. Sua função principal encontra-se no bloqueio da síntese de DNA através de inibição da ribonucleotídeo redutase, mantendo as células em fase $S$. É de fácil utilização ${ }_{z}$ com poucos efeitos tóxicos e com um efeito mielossupressor facilmente revertido. $\underline{O}$ uso da hidroxiuréia nos protocolos de tratamento da anemia falciforme $(\overline{\mathbf{A F}})$ teve seu iń́cio na década de $\mathbf{8 0}$ nos pacientes

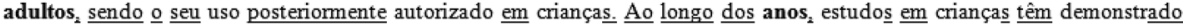
igual eficácia de HU sem grandes efeitos colaterais. Assim. o presente estudo teve como objetivos a partir de uma série de casos, investigar a eficácia e a tolerabilidade ao uso de $\mathrm{HU}$ por pacientes portadores de $\mathrm{SF}$ nas faixas etárias pediátrica adultos jovens, bem como avaliar variações $\underline{\text { de }}$ parâmetros hematológicos em ambos os grupos etários $\underline{\text { e }} \underline{\text { dos }}$ valores $\underline{\text { da }}$ $\mathrm{HbF}_{\mathrm{z}}$ no grupo pediátrico.

Regular font $=$ conceptual encoding (100 units)

Underlined $\equiv$ procedural encoding (206 units)

Bold = conceptual encoding working as procedural encoding (35 units)

(253 words / Type-token ratio $=0.62$ ) 


\title{
The role of archival and manuscript research in the investigation of translator decision-making
}

\author{
Jeremy Munday \\ University of Leeds, UK
}

\begin{abstract}
This chapter discusses the application of research methodologies from history and literary studies to the analysis of the translation process. Specifically, this concerns the use of literary archive and manuscript material to investigate the various stages in the construction of the translation product. Such material has been drastically underexploited in translation studies to date. The chapter describes the type of material available for researchers and how this has been used. This is followed by a case study involving the detailed textual analysis of a translator's drafts and revisions. The chapter considers the value of such research methods in investigating the translation process and how they might complement and interact with other methodologies.
\end{abstract}

Keywords: descriptive translation studies, draft translations, literary manuscripts, publishers archives, research methodology, translator papers, translation process, translation product

\section{Introduction}

This chapter discusses a process-oriented methodology based on primary sources that have very often been overlooked in translation studies: the analysis of literary translator papers, manuscripts and archives which give potentially unrivalled insights into translator decision-making. This type of analysis brings into play research methodologies from history and literary studies. The original purpose of the material is distinct from its role as an object of translational study, bearing out what Overbeck $(1993,63)$ says in relation to literary manuscripts: "[literary] scholars value such records for their primary or secondary relationship to study of the literary text, while the owner may view them simply as a record of business transacted." Historians consider that such primary sources "are essentially 'results' 
or 'traces' or 'relics' or 'tracks' of historical activity" (Grigg 1991, 231). In translation studies, they reveal some of the normally hidden traces of translatorial activity and are a real-time record of some of the translator's decision-making processes.

Archive material facilitates the reconstruction of translational norms and provides a bridge between what, for Toury $(1995,65)$, are the two major sources for their study:

1. Textual sources: the published target texts (TTs), as well as catalogues of TTs for preliminary norms. These he describes as "primary products of norm-regulated behavior".

2. Extratextual sources: notably "statements made by translators, editors, publishers, and other persons involved in or connected with the activity". These are "by-products of the existence and activity of norms" and are "partial and biased, and should therefore be treated with every possible circumspection" (Toury 1995, 65).

In my opinion, Toury's dismissal of extratextual sources is unjustified. It may be true that there is a strong element of self-justification in some pronouncements (see, for instance, the book-length contributions from Levine 1991 and di Giovanni 2003) and it is clear that some paratexts that accompany a translation, such as translator prefaces, may attempt to control the reception of the TT (Dimitriu 2003). However, in most cases there is surely no "deliberate desire to mislead and deceive" (Toury $1995,66)$ and, even if there were, we should certainly not ignore what the translators say. The window these pronouncements provide into the working practice of the translator may be unobtainable through other means.

However, my contention is that just as valuable in this regard are draft manuscripts and papers. These are unpublished primary textual sources, preceding and building to the TT itself. They are interim products which offer crucial and more direct access to the creative process that is literary translation and provide written evidence of the translator's decision-making. The present chapter will focus precisely on the possibilities provided by the textual analysis of literary drafts, evaluating previous work in this area and, through a case study, illustrating the wealth of detail they may offer. It should be stressed that this is not a typical form of analysis in translation process research, with its tendency to focus more on experimental methodologies, often accompanied by quantitative statistical analysis. Equally crucially, the analysis of drafts brings into play a new and strong interdisciplinary element in its meshing with analysis from a literary studies tradition. Hansen (2010) calls this "the liberal arts paradigm". It is so closely involved in the study and interpretation of texts that, without it, "in empirical research into translation or translation processes it is impossible to achieve reasonable results" (Hansen 2010, 203). 


\section{Archive, manuscripts and personal papers}

It is important to distinguish between these three terms, differentiated by the Society of American Archivists as follows (Pearce-Moss 2005, online). An archive comprises " $[\mathrm{m}]$ aterials created or received by a person, family, or organization, public or private, in the conduct of their affairs [...]; permanent records". A manuscript is: "1. A handwritten document. - 2. An unpublished document. -3. An author's draft of a book, article, or other work submitted for publication." Personal papers are: "Documents created, acquired, or received by an individual in the course of his or her affairs and preserved in their original order (if such order exists)." For literary translation studies purposes, the most fruitful archives are generally state censorship files or the business records of a publisher. These will typically include details of the commissioning and production of individual books and series, contracts, costs, sales figures, and so on. Of particular interest is the correspondence between editors and individual translators which may contain comments and queries on the titles as well as details of the working conditions. For instance, the Allen \& Unwin archive in the Archive of British Publishing and Printing at Reading, UK, provides a comprehensive picture of the relations between publisher and translators over many years. To give just one example, Bernard Miall, translator and reader, worked for them from 1914 until his death in 1953. His letters, every two or three days for much of that period, graphically recount his struggle to make a living (Munday, forthcoming).

Draft manuscripts, as defined above, are often rare in publishers' archives as they tended to be discarded once a book had been published. None of Miall's seem to survive, while in the Penguin Classics archive in Bristol the notable exception is Stanley Handford's 1971 retranslation of Tacitus's Agricola and Germania (Tacitus 1970), only retained because of a legal case brought by the estate of the original translator, Harold Mattingly (see Munday 2012, Chapter 4). Such manuscripts are more likely to be found amongst author or translator papers, an indication perhaps of the higher value that these actors attach to them as records of a creative process. These contain drafts marked up by the authors and/or their translators and editors as well as correspondence answering specific queries (see Munday 2012, Chapter 4). Nonetheless, considerable investigative work is needed to uncover their whereabouts, a fact acknowledged by the growth of online resources such as GLAM (http://glam-archives.org.uk/) and the Archives Hub (http://archiveshub. ac.uk/). These papers may contain:

- Correspondence between the translator and the author, editor and agent, organized into files according to correspondent. 
- Query sheets which the translator has sent to the author or editor, or queries sent by a reader to the translator.

- Notebooks, in which the translator may have recorded progress, problems and even snippets of translation.

- Draft manuscripts at different stages of the process, often with handwritten corrections.

- Ephemera or miscellaneous material, such as CVs, publicity for the titles, postcards, etc.

Together, these shed considerable light on the working practices and personal life of an individual translator.

\subsection{Archives, manuscripts and papers in translation studies research}

Access to archives enables a detailed picture to be constructed of the role of translation in concrete socio-historical contexts. To give just two recent examples, the papers in Rundle and Sturge (2010) examine translation policy and censorship in mid-twentieth century Europe while Billiani (2007) uncovers the contribution of Einaudi publishers to poetry translation and the construction of a post-Second World War identity in Italy. The analysis of correspondence has allowed investigation of the relationship between Ezra Pound and Paul Blackburn and their translation strategies (Venuti 1995/2008) and an appreciation of the influence of figures such as Edward Garnett, husband of famous translator Constance, in the reception of Russian literature (Smith 2011). However, unless the translator is a wellknown author or self-translator, drafts are less often available. Even when they are, relatively little work from within translation studies has sought to track translator decisions in those drafts. What has been done reveals some of the methodological considerations that arise. Pijuan Vallverdú (2007) analyses a section of the revised typescript drafts of Manuel de Pedrolo's Catalan translation of William Faulkner's Light in August (1932), published in Barcelona by Edicions 62 as Llum d'Agost (1969). The features that are noted in the analysis are classified very broadly as: spelling, syntax, lexis, punctuation, "unnecessary corrections" and "incorrect corrections" (Pijuan Vallverdú 2007, 64), but few examples are given. More detail is provided by translator Peter Bush $(2006,27)$, who presents "the writing process of a translation" by describing the evolution of a paragraph from the opening of his own translation of Spanish novelist Juan Goytisolo's Carajicomedia (2000). ${ }^{1}$ The study examines what Bush calls his "first draft" and "sixth draft". Between these two, the basic structure remains consistent despite modifications, which amount

1. Published as A Cock-Eyed Comedy (Serpent's Tail, 2002). 
to the translation of names, the replacement of synonyms and some reworking of syntax. Bush's account of his motivations adds to our understanding of the reasons behind certain changes. For example, he indicates that the shift from pounding the carpet to pounding the parquet was a deliberate move to intensify the alliteration while later decisions between the sixth and eighth drafts concerned strategic matters such as how to deal with heteroglossia (French and Latin expressions in the Spanish ST). However, his analysis leaves crucial unanswered questions including the cognitive processes which preceded the typing of the first draft and the order in which changes were made in the intervening and absent drafts.

In an attempt to answer such questions, Jones (2006) combines open-ended interviews with five poetry translators about their background and translation strategies with a think-aloud protocol (TAP) study of himself translating a SerboCroat poem through four drafts. The findings of the think-aloud protocol are classified into 'sequences' (strategic and problem-solving moves), 'foci' (the type of problem featuring in each sequence, the most frequent of which were lexis, image, rhythm, and rhyme) and 'drafts' (a quantitative analysis of the predominant characteristics of each draft). In the latter, Jones $(2006,70)$ sees a statistically significant shift from lexis foci in Draft 1 to rhythm, rhyme and poetic form in Draft 2 to a more holistic revision in Drafts 3 and 4. We shall return to this below.

Obviously, the study of drafts is objectively more solid if it is carried out by a third party. Filippakopoulou (2008) analyses the drafts and comments of a translation partnership: Ros Schwartz and Lulu Norman's translation of Aziz Chouaki's novel L'Etoile d'Alger (2002). ${ }^{2}$ She also discusses the translators' self-reflective article on this collaboration (Schwartz and Norman 2006) and argues for the complementarity of the two sources: the drafts give "voyeuristic" access to the normally concealed agency of the translator, to the revisions, corrections and prescriptive quest for linguistic accuracy; the retrospective protocol, in which Schwartz and Norman seem more confident, "speaks about the emotive experience that arguably is the enterprise of translation" (Filippakopoulou 2008, 34).

Filippakopoulou importantly notes methodological problems associated with the analysis of drafts. These are "messy documents [...] loose sheets of paper, designed to serve a short-term purpose" $(2008,28)$, typed pages covered with handwritten corrections, suggestions, queries and musings. Although she does include three copied pages of notes for illustration, she does not go much further in the analysis of the patterns than to note general categories of shift (e.g., "changes in word order; changes in verb perspective; punctuation replacing conjunctions; translation shifts and adaptations...") and to claim that the notes in the drafts "resist [...] standard philological/literary analysis" $(2008,28)$. We shall begin to tackle

2. It appeared in English as The Star of Algiers (Serpent's Tail, 2006). 
this in more depth in the case study through meticulous analysis of a small section of text through multiple drafts in order to reconstruct the translator's actions at different points and to posit the decision-making processes underlying these. What we are thus proposing is the bringing together of product analysis with a study and deduction of process. In some ways this follows the interdisciplinary methodology proposed by Alves et al. (2010) with its combination of corpusbased and process-based approaches.

\section{Case study - drafts of Bellos's retranslation of Perec's Les choses ${ }^{3}$}

David Bellos, now Professor of French and Comparative Literature at the University of Princeton, is the prominent translator of the French experimental writer Georges Perec (1936-1982) and the Albanian novelist Ismail Kadare (b. 1936). After his successful translation of Perec's masterpiece Life: A user's manual ${ }^{4}$ (see the analysis in Munday 2012, Chapter 4), which brought Perec to greater international attention, Bellos revised Helen Lane's earlier translation of Perec's Les choses: une histoire des années soixante (1965). The Bellos papers at the University of East Anglia contain Bellos's notebooks and other material, including draft manuscripts, related to these translations. In the case of Les choses, a notebook itself contains the draft of his revision of the first half of the book. ${ }^{5}$ This starts in the form of amendments to a printed copy of Lane's text, but after just two pages Bellos seems to have decided that so much revision was needed that it was preferable to write out a totally new version by hand. ${ }^{6}$

The small sample of the papers consulted for this study comprises:

1. Lane's published TT (Perec 1967), cut out and pasted by Bellos onto the lefthand side of sheets of squared paper.

2. Draft 1: Bellos's first draft of handwritten revisions to the opening two printed pages of Lane's translation. These revisions are in pen and pencil. It can be presumed that these were done at different times since those in pencil, which

3. I am grateful to: the University of East Anglia Special Collections for their assistance in this case study; to the Universityof Leeds and AHRC for funding my research leave during 2010; and to David Bellos for an interview in Princeton in November 2010 and for granting permission to quote from his chapters for the purpose of this chapter.

4. Published by David R. Goldine in 1987.

5. David Bellos papers, University of East Anglia, Norwich, UK. Box LIT/TA/DB/3, notebook dated 'Sept - 10.12.1988'

6. Since confirmed by Bellos himself (personal communication). 
we shall call Draft 2, are much more substantial and often involve a complete rewriting of Draft 1.

3. Draft 3: Bellos's later draft of the first half of the text, together with further revisions. This draft was written in pen on a new sheet of paper (see Appendix 1). 4. The published Bellos TT (Perec 1990).

What we are most concerned with are revisions made at different stages. These indicate an evaluation by the translator that causes him to make a change to the text and, in the case of multiple revisions, suggest what Angelone $(2010,18)$ calls 'uncertainty' related to a particular 'problem nexus.' The drafts make this uncertainty observable in the form of multiple written amendments. Analysis of the very first paragraph of the book shows the huge amount of micro-data that may be generated by just a small section of text:

\section{Perec ST (Perec 1965:9)}

L'œil, d'abord, glisserait sur la moquette grise d'un long corridor, haut et étroit. Les murs seraient des placards de bois clair, dont les ferrures de cuivre luiraient. Trois gravures, représentant l'une Thunderbird, vainqueur à Epsom, l'autre un navire à aubes, le Ville-de-Montereau, la troisième une locomotive de Stephenson, mèneraient à une tenture de cuir, retenue par de gros anneaux de bois noir veiné, et qu'un simple geste suffirait à faire glisser. La moquette, alors, laisserait place à un parquet presque jaune, que trois tapis aux couleurs éteintes recouvriraient partiellement.

\section{Bellos Draft 1 (showing his revisions to Lane's published TT)}

The eye, at first, would sglide over the greay rug of a long corridor, high and wooden fitted cupboards, light and gleaming

narrow. The walls would befeabinets, whose copper fittings would gleam

with copper fittings. Three engravings - one representing Thunderbird, the winner

at Epsom, another a paddlewheel steamer, the "Ville-de-Montereau," the third a

Stephenson locomotive - would lead to a leather curtain, hanging from large rings

of black veined grainy black wood, that a simple mere gesture would suffice to Then

slide back. [Fthe rug, then, would give way to an almost yellow parquet floor,

soft-hued

which threeprugs in softers would partially cover.

7. "Uncertainty is defined here as a cognitive state of indecision that may be marked by a distinct class of behaviors occurring during the translation process" (Angelone 2010, 18). 
The source text (ST) is not especially complicated and Lane's translation was more or less literal. Notable only are her confusion of moquette (which should be fitted carpet rather than rug), the omission of an equivalent for de bois clair ('of light wood') and the translation of retenue par de gros anneaux ('held by large rings') as hanging from large rings. Bellos's first draft makes ten changes in a paragraph of 90 TT words. These are generally minor, involving replacements on the lexical level (glide over > slide over; US gray > UK grey; cabinets > fitted cupboards; paddle wheel steamer > paddle steamer; a simple gesture > a mere gesture), word order and syntactic structure (black-veined wood > grainy black wood; in soft colors > soft-hued; The rug, then,... > Then the rug...) and the rectification of Lane's omission, which leads to a rewording of the clause (cabinets, whose copper fittings would gleam > wooden fitted cupboards, light and gleaming with copper fittings).

More substantial revisions, which perhaps more keenly reveal the decisionmaking processes, can be seen in the amendments in Draft 2, written in pencil beside and below the first draft. It would seem that these were added later since three whole sections of the paragraph, covering most of the first three sentences, are re-written in full and provide the basis for the subsequent drafts.

\title{
Bellos Draft 2
}

\begin{abstract}
Your eye, first of all, would slide over the grey carpet in the a high, narrow, long corridor. The walls would be made of fitted cupboards of light-coloured wood with gleaming brass fittings. Three prints, one depicting Thunderbird, the Epsom winner, another a paddle steamer, the VdM and the third a Stephenson loco, would lead to a leather curtain, hanging from large rings of black-veined grainy black wood, which would slide back at the merest movement of the an arm. Then the carpet would give way to an almost yellow woodblock floor, which three faded carpets would partly cover.
\end{abstract}

Comparison of Draft 2 with Draft 1 shows that the changes are related to lexis, syntactic restructuring and, a new element, cohesive devices:

Lexis: first > first of all; rug > carpet (twice); Three engravings $>$ Three prints; representing > depicting; parquet floor $>$ woodblock floor; soft-hued rugs $>$ faded rugs; partially > partly. Of these, the translation carpet is a correction (see above). The others are near-synonyms or slight adjustments to meaning.

Syntactic restructuring: long corridor, high and narrow > high, narrow, long corridor; the winner at Epsom > the Epsom winner; the walls would be wooden fitted cupboards, light and gleaming with copper fittings $>$ the walls would be made of fitted cupboards of light-coloured wood with gleaming brass fittings; that a mere gesture would suffice to slide back > which would slide back at the merest movement of 
an arm. The examples are frequent, showing that restructuring plays an important role in decision-making at the second draft stage. That these occur after the initial revision suggests that the decision to opt for major restructuring is the result of the translator's dissatisfaction with low-level lexical modification.

Cohesive devices: The eye $>$ Your eye; $a>$ the $>a$ (high... corridor); the third $>$ and the third. It is interesting that the translator should focus on the detail of deixis, which may be quite crucial to the depiction of the narrative point of view (see Mason and Serban 2003). All three examples of cohesive devices indicate a move away from literal translation towards a more conventional English, especially the change from The eye to the more specific Your eye. This is a typical translation of the French definite article used with a part of the body, but it necessarily defines the narrative perspective. The concentration on higher-level considerations is supported by an extratextual source: Bellos (2001) has written about the particular problem of translating verbs in Perec's prose, and the importance, in these lines of Les choses, of the French conditional (glisserait... seraient... mèneraient... and so on) to create a dream-like sequence that needs to be retained in the translation.

Draft 3 is also handwritten, as can be seen in Appendix 1. A classification of the revisions compared to Draft 2 is given in Appendix 2 and again shows a predominance of syntactic restructuring.

This is also the case with subsequent handwritten amendments made to Draft 3 (see Appendix 1): syntactic restructuring is dominant, followed by cohesive devices, but with only one additional lexical revision: the Epsom winner > the Derby winner. What is very noticeable is that many of these amendments to the text concern the very same points that had been revised at earlier stages. These recurrent doubts are what I term "critical points" for the translator. They are similar to the concept of "rich points" (PACTE 2011,37) but are identified on the basis of translator behaviour rather than selected a priori as a test item. Thus, a critical point is one where each translation draft revisits and further explores the same problem. For example, the translation of the French conditional suffirait à faire glisser shows an oscillation in modality (would slide $>$ could slide $>$ would slide). Similarly, Bellos's attempts to personalize the first sentence by making the subject of the action the pronoun you rather than your eye (What you would see first of all would be...) is rejected in favour of the choice from Draft 2 (Your eye, first of all, would glide over...). The order of epithets is a particular preoccupation of the translator, with constant revision around corridor, cupboards and fittings. Here, the concern seems to be with achieving a 'natural' order and rhythm in the English.

The amended Draft 3 was itself revised at one final point prior to publication (Perec 1990): a paddle-steamer called The City of Montereau becomes a paddlesteamer named Ville-de-Montereau. Called and named are synonymous, but the retention of the French name for the ship may have come from a decision to retain 
foreign elements where possible, which would be comparable to the late decision on how to deal with names in Bush (2006), above.

Some may find such analysis sterile and repetitive, but it is only by examining in such detail that we can move on from impressionistic comments and truly begin to understand the processes involved at each stage. Failure to do so, or the cherry-picking selection of a more "interesting" extract, betrays a lack of investigative rigour. It is beyond doubt illuminating to see the types of revisions made at each stage in the process, even in a small sample of one paragraph. Bellos's Draft 1 revision of Lane's literal translation centres on the lexical level and syntactic reordering at the phrase level; Draft 2, where Bellos completely rewrites the text, shifts the focus to syntactic and structural change and introduces a new element in cohesive devices, which is repeated in Draft 3. One clear feature is that lexical revision decreases during the revision process, particularly between Drafts 2 and 3. Overall, revision reduces dramatically as the final published version is approached, although the major change at that final stage is in fact quite a strategic one since it involves the choice between borrowing and literal translation of a proper name, a culture-specific element. In part, this seems to chime with the findings from Jones's (2006) TAP study into drafts of his own translation of a poem, where lexical problems gave way to form and structure and then a holistic evaluation (see above). It is a finding worthy of further investigation.

\section{Questions of research methodology}

As useful as descriptive translation studies are for the identification of trends of translation shifts and translational norms in published texts, their scope is inevitably limited if they do not seek to combine analysis of the translated product with an investigation of the translation process. For literary translation, the existence of author and/or translator papers, manuscripts and drafts offers just such an insight into decision-making. The advantages are manifold: analysis of drafts by a thirdparty analyst reduces subjectivity compared to those descriptions of the process by the translators themselves; multiple drafts give concrete information on decisionmaking at different stages in the translation process; the existence of other material, such as query lists, reader reports and, most particularly, correspondence, may provide clear evidence about the negotiation and location of power in the publishing world. In this chapter I have concentrated mainly on the possibilities offered by the detailed textual study of drafts. It should be stressed that these preliminary findings will only really be interpretable in the context of other similar studies which will enable comparisons to be made across translators and projects. Close analysis of Bellos's papers as a whole shows that he seems to be much more 
systematic and organized than many other translators. But then those translators whose papers are available in libraries are those who have carefully retained the material over a number of years. By contrast, many of Helen Lane's papers were eaten by mice in her house in rural France. The actual organization of the papers by the library archivists also imposes some order on the collection that may not have been previously contemplated. Importantly, though, the findings from such studies should complement and be complemented by other, more experimental, research methods into translation processes in order to achieve triangulation (Alves 2003). These should include the study of translator correspondence, interviews and think-aloud protocols, corpus-based studies of texts and translator choices, and perhaps also keystroke logging and eye-tracking studies.

The drafts should be seen as real-time and real-world evidence of translation revisions and doubts, sometimes with a rationale for decision-making. They constitute visible traces of the translatorial act. They are also tangible objects that create a direct link to the creation of some of the great works of literature. Their physical form is crucial to their interpretation, since handwriting needs to be deciphered, the type of paper and ink may be significant, and so on (Prescott 2008). Touching the paper and seeing the ink used by the translators also puts the scholar into the closest contact with great translators. It is a physical sensation. For me, the archive allows the researcher literally to feel and smell the presence of literary creation.

Developments in modern communication will mean that this is bound to change. Derrida (1996) already saw this with the advent of e-mail. Hard copies of electronic communication (fax and e-mail) are now present in some existing repositories, such as the Andrew Hurley papers at the Harry Ransom Center, Austin, Texas. It is quite conceivable that future collections of author and translator "papers" will predominantly, or even completely, comprise digital communications and will be widely available online. Unless translators are made aware of their importance, there is the risk that multiple early drafts may no longer be retained, since the default save facility of word processing packages automatically overwrites the previous version of the document. However, the upside is that more documents are likely to be made available, including detailed e-mail correspondence, and they will be electronically searchable. This will hugely increase the potential for investigation and will doubtless benefit from interdisciplinary co-operation using analytical tools from corpus linguistics, for example. In addition, translator statements and discussion have even now shifted to blogs (e.g., http://www. booktrust.org.uk/books-and-reading/translated-fiction/) and online forums such as KudoZ ${ }^{\mathrm{TM}}$ (http://www.proz.com/kudoz/) where translation problems and solutions are discussed in their thousands. In this way, more and more traces of the translatorial act and decision-making will become available in the public domain and will provide rich material for analysis. 


\section{References}

Alves, Fabio (ed). 2003. Triangulating Translation. Amsterdam: John Benjamins. DOI: $10.1075 /$ btl. 45

Alves, Fabio, Adriana Pagano, Stella Neumann, Erich Steiner, and Silvia Hansen-Schirra. 2010. "Translation Units and Grammatical Shifts: Towards an Integration of Product- and Process-Based Translation Research.” In Translation and Cognition, ed. by Gregory M. Shreve, and Erik Angelone, 109-142. Amsterdam: John Benjamins.

DOI: 10.1075/ata.xv.07alv

Angelone, Erik. 2010. "Uncertainty, Uncertainty Management and Metacognitive Problem Solving in the Translation Task." In Translation and Cognition, ed. by Gregory M. Shreve, and Erik Angelone, 17-40. Amsterdam: John Benjamins. DOI: 10.1075/ata.xv.03ang

Bellos, David. 2001. “Putting Grammar to Work: Tense and Mood in Perec's Prose." Sites 5 (1): 283-298.

Billiani, Francesca. 2007. "Renewing a Literary Culture through Translation: Poetry in Post-War Italy." In Translation as Intervention, ed. by Jeremy Munday, 138-160. London: Continuum.

Bush, Peter. 2006. “The Writer of Translations." In The Translator as Writer, ed. by Peter Bush, and Susan Bassnett, 23-32. London: Continuum.

Derrida, Jacques. 1996. Archive Fever: A Freudian Impression. Translated by Eric Prenowitz. Chicago: University of Chicago Press. DOI: 10.2307/465144

di Giovanni, Norman Thomas. 2003. The Lesson of the Master: On Borges and his Work. LondonNew York: Continuum.

Dimitriu, Rodica. 2003. “Translators' Prefaces as Documentary Sources for Translation Studies.” Perspectives: Studies in Translatology 17 (3): 193-206. DOI: 10.1080/09076760903255304

Filippakopoulou, Maria. 2008. “Translation Drafts and the Translating Self.” In Translating Selves: Experience and Identity between Languages and Literatures, ed. by Paschalis Nikolaou, and Maria-Venetia Kyritsi, 19-36. London: Continuum.

Grigg, Susan. 1991. "Archival Practice and the Foundations of Historical Method.” The Journal of American History 78 (1): 228-239. DOI: 10.2307/2078095

Hansen, Gyde. 2010. "Integrative Description of Translation Processes." In Translation and Cognition, ed. by Gregory M. Shreve, and Erik Angelone, 189-211. Amsterdam: John Benjamins. DOI: 10.1075/ata.xv.11han

Jones, Francis. 2006. “Unlocking the Black Box: Researching Poetry Translation Processes.” In Translation and Creativity: Perspectives on Creative Writing and Translation Studies, ed. by Manuela Perteghella, and Eugenia Loffredo, 59-74. London: Continuum.

Levine, Suzanne Jill. 1991. The Subversive Scribe: Translating Latin American Fiction. St Paul, MN: Graywolf Press.

Mason, Ian, and Adriana Şerban. 2003. "Deixis as an Interactive Feature of Literary Translations from Romanian into English”. Target 15 (2): 269-94. DOI: 10.1075/target.15.2.04mas

Munday, Jeremy. 2012. Evaluation in Translation: Critical Points of Translator Decision-Making. Abingdon: Routledge.

Munday, Jeremy. Forthcoming. "Translator Training and Identity." In The Oxford History of Literary Translation in English: Volume V, The Twentieth Century, ed. by Lawrence Venuti, Oxford: Oxford University Press.

Overbeck, Lois More. 1993. "Researching Literary Manuscripts: A Scholar's Perspective." The American Archivist 56 (1): 62-69. 
PACTE Group. 2011. "Results of the Validation of the PACTE Translation Competence Model: Translation Project and Dynamic Translation Index.” In Cognitive Explorations of Translation, ed. by Sharon O'Brien, 30-56. London: Continuum.

Pearce-Moss, Richard. 2005. A Glossary of Archival and Records Terminology. Chicago: The Society of American Archivists. http://www.archivists.org/glossary/index.asp.

Perec, Georges. 1965. Les choses: une histoire des années soixante. Paris: René Juillard.

Perec, Georges. 1967. Les choses: A Story of the Sixties. Translated by Helen Lane. New York: Grove Press.

Perec, Georges. 1990. Things: A Story of the Sixties. Translated by David Bellos. London: Collins Harvill.

Pijuan Vallverdú, Alba. 2007. "Anàlisi del mecanoscrit i la correcció de la traducció de Manuel de Pedrolo de Llum d'agost." Quaderns 14: 57-66.

Prescott, Andrew. 2008. “The Imaging of Historical Documents." In The Virtual Representation of the Past, ed. by Mark Greengrass, and Lorn M. Hughes, 7-22. London: Ashgate.

Rundle, Christopher, and Kate Sturge (eds). 2010. Translation Under Fascism. Basingstoke: Palgrave MacMillan. DOI: 10.1057/9780230292444

Schwartz, Ros, and Lulu Norman. 2006. "How Was it for You?" In Other Words 27: 28-34.

Smith, Helen. 2011. "Edward Garnett: Interpreting the Russians." Translation and Literature 20 (3): 301-313. DOI: 10.3366/tal.2011.0033

Tacticus, Cornelius. 1970. Agricola and Germania. Translated by Harold Mattingly, revised by Stanley Handford with an introduction by Harold Mattingly. Harmondsworth: Penguin.

Toury, Gideon. 1995. Descriptive Translation Studies - and Beyond. Amsterdam: John Benjamins. DOI: $10.1075 /$ btl.100

Venuti, Lawrence. 1995/2008. The Translator's Invisibility: A History of Translation. London: Routledge. DOI: 10.4324/9780203360064 
Appendix 1. Photocopy of Bellos's handwritten Draft 3, with amendments (reproduced with the permission of David Bellos).

\section{RART ONE}

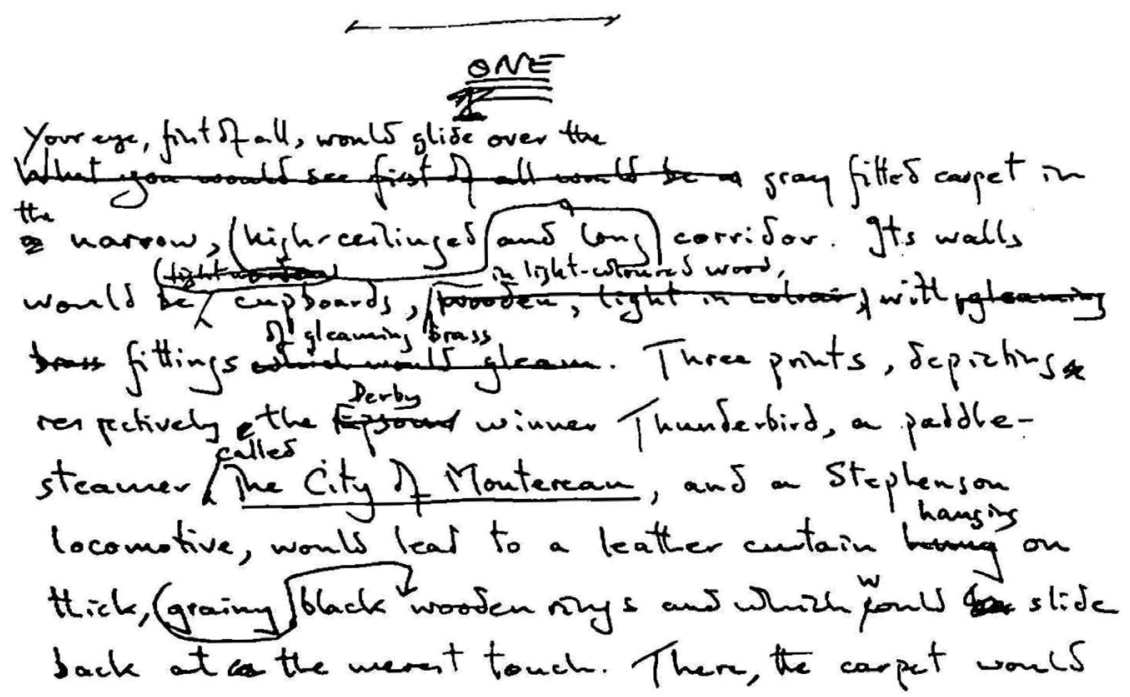

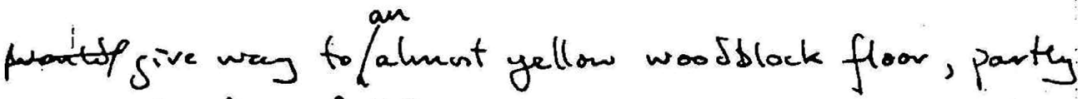
coveres by three fajed rugs.

$+$

Appendix 2. Classification of initial revisions at Draft 3 stage

Lexis: slide over > glide over; grey carpet > gray, fitted carpet; fitted cupboards > cupboards; hanging $>$ hung; at the merest movement of an arm > at the merest touch

Syntactic restructuring: Your eye, first of all, would slide over... > What you would see first of all would be...; high, narrow, long corridor > narrow, high-ceilinged and long corridor; Thunderbird, the Epsom winner > the Epsom winner Thunderbird; fitted cupboards of light-coloured wood with gleaming brass fittings > cupboards, wooden, light in colour, with gleaming brass fittings; which three .. rugs... would partly cover $>$ partly covered by three... rugs.

Cohesive devices: of a... corridor > in a ... corridor; the walls > its walls; would be made of $>$ would be; $\varnothing>$ respectively; Then, the carpet would... > There, the carpet would...

Modality: would slide $>$ could slide. 


\title{
Sound effects in translation
}

\author{
Inger M. Mees, Barbara Dragsted, Inge Gorm Hansen and \\ Arnt Lykke Jakobsen \\ Copenhagen Business School (CBS), Denmark
}

\begin{abstract}
On the basis of a pilot study using speech recognition (SR) software, this chapter attempts to illustrate the benefits of adopting an interdisciplinary approach in translator training. It shows how the collaboration between phoneticians, translators and interpreters can (1) advance research, (2) have implications for the curriculum, (3) be pedagogically motivating, and (4) prepare students for employing translation technology in their future practice as translators. In a twophase study in which $14 \mathrm{MA}$ students translated texts in three modalities (sight, written, and oral translation using an SR program), Translog was employed to measure task times. The quality of the products was assessed by three experienced translators, and the number and types of misrecognitions were identified by a phonetician. Results indicate that SR translation provides a potentially useful supplement to written translation, or indeed an alternative to it.
\end{abstract}

Keywords: written translation, sight translation, oral translation, speech recognition, pronunciation, productivity, translation modalities

\section{Introduction}

Consider the following three scenarios.

\section{Scenario 1}

Peter has just broken his arm and has been told by the doctors that he can't use it for six weeks. This is particularly inconvenient because he makes his living as a translator and depends on being able to type. He could dictate his translations but would then have to hire a secretary to type them, which is expensive. In addition, he likes making revisions as he goes along. He is working on a translation of an annual report and has several major projects in the pipeline. What can Peter do to get his translations done on time? 


\section{Scenario 2}

Emma is a talented language student specialising in translation and interpreting. Along with those courses, the curriculum also requires her to take other language subjects, including courses in linguistics, such as phonetics. "It's so boring", says Emma, "Why do we have to learn all this useless stuff about consonant articulation, vowel length, weak and contracted forms, and differences between Danish and English vowels? When I'm abroad I can communicate with English-speaking people without any problem whatsoever." Emma wants more "relevant" subjects which can assist her in her future career. What can the university do to motivate students like Emma, who likes the practical components of the translation and interpreting programme, but finds it difficult to see the point of subjects such as phonetics?

\section{Scenario 3}

David is a full professor of marketing and consumer behaviour at a Danish university, where he has recently become director of a research centre with a global network. He is delighted to have received funding for a large-scale project involving researchers from many countries. In future, David's working language will be English, and all his articles and reports will have to appear in English. David is a very proficient speaker of English, with a wide vocabulary and native-like pronunciation, but writing poses a major obstacle. For despite his many talents, David has one great handicap: he is dyslexic. How can David be helped?

For these three scenarios, based on real life cases (names have been changed), one solution could be to introduce speech recognition (SR) software. Peter would be able to dictate his translation and have it converted into a written translation on the spot. Emma would suddenly see the relevance of phonetics. And David would overcome his difficulties with writing in English. Both Peter and David would perhaps save time, and all three might even deliver output of an equally high - or improved - standard, and experience more variation in their work.

Introducing SR into translation (see Jurafsky and Martin 2000, 235-284 for an introduction to SR technology) presupposes the integration of multiple disciplines simultaneously. "Speaking" rather than "writing" a translation itself involves crossing the borders between translation and interpreting since the translation is produced orally, as in interpreting, but is visible on the screen, as in translation (see Gile 1995; 2004; Agrifoglio 2004; and Lambert 2004 for discussions of interpreting and sight translation in comparison with written translation). Thus we are dealing with a hybrid. In addition, it is necessary to draw on the field of phonetics (pronunciation) in order to discover how one can facilitate and improve the dictation of a translation, and specifically how one can reduce the number of misrecognitions to a minimum. To gauge the potential for collaboration, the authors of the present chapter, who represent different areas of research (translation, interpreting and phonetics), decided to embark on an interdisciplinary adventure. 
As stated by Leijten and Van Waes $(2005,741)$, "most research in the field of speech technology has dealt with the technical improvement of speech recognition and the role of phonology". However, more recently there has been interesting work on the applications of speech recognition in a range of other fields, for example, studies on how SR affects the writing process (Leijten and Van Waes 2005), on error correction strategies employed by professional SR users (Leijten, Janssen and Van Waes 2010), and on the use of SR technology in the domain of re-speaking in live subtitling (Luyckx et al. 2010). However, to our knowledge there are no studies such as the one below on the use of SR in translator training.

\section{Methodology}

A two-phase pilot study was undertaken in which we examined the impact on the translation process and product of using an SR system, as compared with typing a translation and producing a normal sight translation.

Two batches of experiments were carried out, the first taking place in March 2010 and the second in December 2010. Sixteen MA Translation \& Interpreting (T\&I) students out of a class of 22 volunteered for the experiments and translated Danish texts into English, their L2, under three different conditions (sight, written, and SR, using the SR software Dragon Naturally Speaking Preferred 10). The data for two participants subsequently had to be discarded owing to technological difficulties, leaving 14 students for analysis. After the first phase in March 2010, half of the participants (experimental group) were asked to work with the SR program at home while the other half was not (control group). In December 2010, the same participants translated comparable texts under the same three conditions. The students did not have access to the Internet, dictionaries or other support. Although this restriction made the set-up less ecologically valid, it was considered a necessary constraint as students would probably have spent more time on information retrieval under the written than the oral conditions, which would have given a skewed picture of the task times in the different translation modes.

In the SR modality, the participants were instructed not to use the keyboard but only to avail themselves of the oral commands. The motivation for this was that three recording programs were running simultaneously during the SR task (keystroke logging, eye tracking and SR), and pilot runs had shown that keyboard activity in the SR task sometimes caused the keystroke software to malfunction. More importantly, if students were allowed to use the keyboard during the SR task, there was the risk that they would revert to typing whenever they encountered problems with the SR system (cf. Leijten, Janssen and Van Waes 2010, 969), 
and this would have defeated the purpose of the exercise. See Dragsted, Mees and Hansen $(2011,16)$ for more details.

Asking the participants to dictate in their L2 is not as curious as it may seem at first sight. In Scandinavia there are "good grounds for referring to English as a second language rather than a foreign language" (Phillipson 2003, 96). Since English and Danish are both Germanic languages exhibiting close correspondences in their sound systems, Danes encounter fewer dictating and pronunciation difficulties than speakers from many other countries. In Denmark, English is taught from age 9 and pervades Danish society (films are subtitled, many companies use English as a lingua franca). Furthermore, since Danish is not a world language, most Danish translators are forced to work bi-directionally if they want to make a living. Translator training in Denmark thus focuses equally on translation in both directions (see Dragsted, Mees and Hansen 2011, 12). Finally, the shortage of English mother tongue translators in the EU makes it increasingly necessary to educate English L2 translators.

The objective of this study was pedagogical, and we wish to state from the outset that the research design can be faulted in several ways. Notably, students' activity between the two data collection phases should have been monitored more carefully (see Sections 2.2 and 3.3). Our study should therefore be regarded as a pilot which can provide preliminary insights and serve as a basis for an improved experimental procedure in a larger study. Nevertheless, despite the flaws in the methodology, there were clear indications that an interdisciplinary approach can be effective in translator training.

\subsection{Research questions and methods of analysis}

The following research questions were addressed and are listed together with the methods of analysis employed in each case. For all three questions, we compared the results for the two phases.

1. What are the differences between task times in the three translation modalities (written, sight, SR)? Translog Audio (Jakobsen and Schou 1999) was used (a) to record oral and written translation output, (b) to investigate transient versions of oral and written translations, and (c) to time the activities.

2. Is there any difference in translation quality in the three modalities? Three experienced teachers/translators were asked to assess the translation quality of the products (see Section 2.2).

3. How many and what type of misrecognitions occur when students translate with SR? Phonetic analyses were performed to identify and categorise the misrecognitions. 
In addition to addressing these issues, which were all measured quantitatively, we conducted retrospective interviews with the participants after the completion of the second experiment in order to gather their impressions of working with an SR system.

\subsection{Procedure}

As stated above, the participants were divided into two groups, an experimental group $(\mathrm{N}=7)$, who were allowed to borrow the SR software and work with it at home, and a control group $(\mathrm{N}=7)$, who did not use the equipment in the interim period. It should be pointed out that, initially, all 14 students trained the SR program as specified by the system. (For a discussion of SR training requirements, see Zong and Seligman 2005, 215-219.) The students in the experimental group were expected to further train the SR system at home and use the software for their translation course assignments and other text production tasks in the interim period, but unfortunately did so in a very limited manner. Consequently, the two groups are very similar in most respects. Therefore the results have been conflated in all the tables below except those where there was a difference between the groups.

Six different text excerpts were selected from the same Danish text (the chairman's statement at the annual general meeting of a bank), each consisting of approximately 110 words. All students translated three texts in the phase 1 experiment and three different texts in phase 2 (total: $14 \times 3 \times 2=84$ translated texts). Every effort was made to ensure that any process/product differences that might emerge across the translation tasks were caused by the translation modality and not by the level of difficulty of the texts. To achieve this, translation tasks were rotated in such a way that four translators produced a written translation of Text A, a sight translation of Text B and an SR translation of Text C; five produced a written translation of Text B, a sight translation of Text C and an SR translation of Text A; and five produced a written translation of Text $\mathrm{C}$, a sight translation of Text $\mathrm{A}$ and an SR translation of Text B.

Three raters - all experienced translators/teachers - were asked to award the translations a score on a scale from 1 to 5,1 being the poorest and 5 the highest quality. The oral translations without SR were transcribed and the transcriptions served as a basis for the evaluations. The transcribers (research assistants) were instructed to ignore temporary solutions and only to write out the final version; punctuation was also added. Thus the raters did not know the modality in which the text had been produced. The task times indicated in Table 2 below refer solely to the time it took to sight translate and not to the subsequent transcription process. 


\section{Analyses}

\subsection{Results for phase 1}

The results for phase 1, discussed in detail in Dragsted, Mees and Hansen (2011), are summarised below.

1. Task times: sight fastest, written slowest. SR takes an intermediate position, but is closer to written than to sight.

2. Quality: highest in written translation (mean: 3.2), somewhat lower in SR (mean: 2.8) and sight (mean: 2.7).

3. Misrecognitions in SR translations: These are caused by (a) hesitations/word boundary problems, (b) homophones, (c) students' incorrect pronunciations, and (d) misrecognitions by the SR system (cf. Derwing, Munro and Carbonaro 2000, 599). The different types are exemplified in Table 1.

Table 1. Examples of misrecognitions identified for one student (S5)

\begin{tabular}{|c|c|c|}
\hline Types of misrecognitions & Intended & Misrecognised as \\
\hline Word boundaries or hesitations & $\begin{array}{l}\ldots \text { offer [ } \text { bfər: }] \text { capital } \\
\ldots \text { a }[ə] \text { credit stimulus } \\
\ldots \text { downward spiral [daunwərd\#s] }\end{array}$ & $\begin{array}{l}\ldots \text { offer a capital } \\
\ldots \text { credit stimulus } \\
\ldots \text { downwards by real }\end{array}$ \\
\hline Homophones & ... i.e. & $\ldots \mathrm{IE}$ \\
\hline Incorrect pronunciations & $\begin{array}{l}\cdots \text { aid [er }[\mathrm{d}] \\
\cdots \underline{\text { of }}[v \mathrm{v}] \mathrm{a}\end{array}$ & $\begin{array}{l}\cdots \text { eight } \\
\cdots \underline{\text { on a }}\end{array}$ \\
\hline Inadequacy of SR software & $\begin{array}{l}\ldots \text { and thus }[\mathrm{d} \partial \Lambda \mathrm{s}] \\
\ldots \text { risk of } \underline{\mathrm{a}}[\partial] \\
\ldots \text { and }[\partial \mathrm{n}] \text { (several) }\end{array}$ & $\begin{array}{l}\ldots \text { and bus } \\
\ldots \text { risk of } \underline{\text { it }} \\
\ldots \text { in (several) }\end{array}$ \\
\hline
\end{tabular}

The examples in Table 1 have been extracted from the SR process of one of the students (S5), who produced all error types within a single translation. The first example is a word boundary problem. The participant pronounces final $r$ in offer (capital), which is unacceptable for non-rhotic Standard British English, that being the option he chose in the SR program. More importantly perhaps, he prolongs the $r$ sound somewhat. This we have categorised as a type of hesitation, since it is a manifestation of his trying to gain time while considering the next word. The lengthening of the sound leads the software into believing that he has said an additional word, namely the indefinite article $a$. Interestingly, there is also evidence that the program may interpret the indefinite article as a hesitation ( $a$ and uh sound identical) and therefore delete it (for example, a credit stimulus was registered as credit stimulus). The software sometimes finds it difficult to delineate the boundaries between words. S5 says downward spiral, but the equipment picks this 
up as downwards by real. (When /p/ is preceded by /s/ in syllable-initial sequences, it is pronounced similarly to /b/, Cruttenden 1994, 46, 140; Collins and Mees 2013,76 .) A second error category is constituted by homophones. The participant dictates i.e., but the program does not realise that he intends the abbreviation for that is and registers the utterance as $I E$.

Both of the categories we have mentioned above would presumably also be a potential source of error for native speakers though this was not tested. The third category is restricted to non-native speakers, namely incorrect pronunciations. The speaker pronounces the word aid with a final /d/ which is slightly too devoiced and which therefore sounds like / $t$ /, and also with a vowel that is slightly too short. In English, vowels are shortened before voiceless consonants (e.g. /t/) and have full length before voiced consonants (e.g. /d/) (Cruttenden 1994, 141), and consequently the program hears his pronunciation of aid as eight. (The student subsequently rectifies this, after which the word is identified correctly.) The word of is pronounced by $\mathrm{S} 5$ with a full vowel rather than a reduced vowel (that is, as a strong form rather than a weak form; Wells 2008, 891) and, as a result, the software misinterprets it as on (which has no weak form). In addition to these three categories, there are a number of misrecognitions for which we cannot easily find an explanation, and which we have therefore categorised as "inadequacy of the SR software". When the participant says and thus, it is identified as and bus, risk of $a$ is interpreted as risk of it, while and is perceived as in.

Our findings in phase 1 led to the following hypothesis for phase 2: With more practice, SR task times will approach those of sight translation, and SR quality will approach that of written translation. In addition, it is expected that the number of misrecognitions will decrease.

3.2 Comparison of results for phases 1 and 2 (March 2010 vs. December 2010)

\subsubsection{Time and quality (Research questions 1 and 2)}

Table 2 shows means of the task times and quality ratings.

Table 2. Time and quality (means of 14 students) - phases 1 and 2 compared

\begin{tabular}{llll}
\hline Phase 1 & Sight translation & SR translation & Written translation \\
\hline Task time (min:sec) & $03: 44$ & $08: 28$ & $11: 07$ \\
Quality rating (from 1-5) & 2.7 & 2.8 & 3.2 \\
\hline Phase 2 & Sight translation & SR translation & Written translation \\
\hline Task time (min:sec) & $03: 40$ & $11: 02$ & $11: 55$ \\
Quality rating (from 1-5) & 2.5 & 2.8 & 2.7 \\
\hline
\end{tabular}


In phase 2, sight translation remains the quickest. The mean task times for the written translation are also more or less unchanged. However, our prediction that SR translation would become faster was not confirmed, since the SR translation in phase 2 on average took longer than that in phase 1; in fact, it took almost as long as the written translation.

Figures 1 and 2 show the task time patterns for the individual participants. (S1, S5, S6, S8, S10, S11, S12 were the students who were asked to use the program at home.) It can be seen that, in phase 1, only two students (S2 and S11) were slower at producing the SR translations than the written translations. Contrary to our predictions, this increased to six of the 14 translators (S2, S3, S7, S8, S10, S11) in phase 2, with one of the students (S10) in the experimental group being particularly slow. Learning to use the commands resulted in her spending much time correcting a few isolated mistakes. Excluding this participant, the mean task times are: Sight 3:42; SR 10:02; Written 12:04.

The quality ratings for written translation and SR became more similar, as predicted, although it should be noted that the convergence was owing to written translation ratings becoming lower rather than those for SR translation becoming higher. In phase 1, three out of 14 students produced better SR translations than written translations, while in the second round this was the case for six participants. The quality of the sight translation output was still the poorest. As the sight translations were transcribed by research assistants after the experiment, these texts may have been more correct with respect to spelling and punctuation than the translations produced by the same students in the other modalities. The quality ratings may therefore have been slightly better than would otherwise have been the case. However, as the sight translations received the lowest scores, this factor would only have further increased the differences between the modalities.

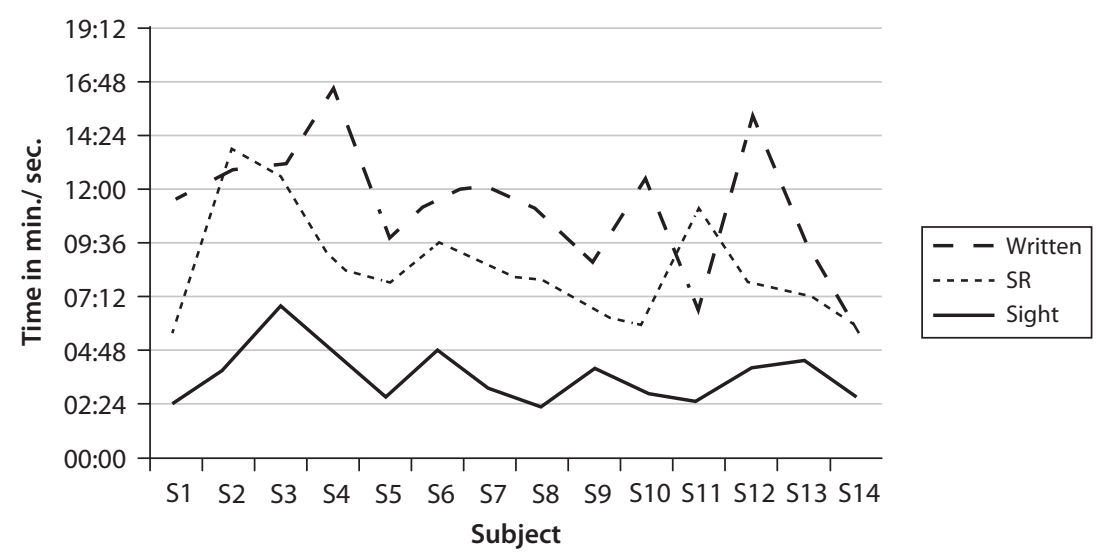

Figure 1. Individual task times in phase 1 


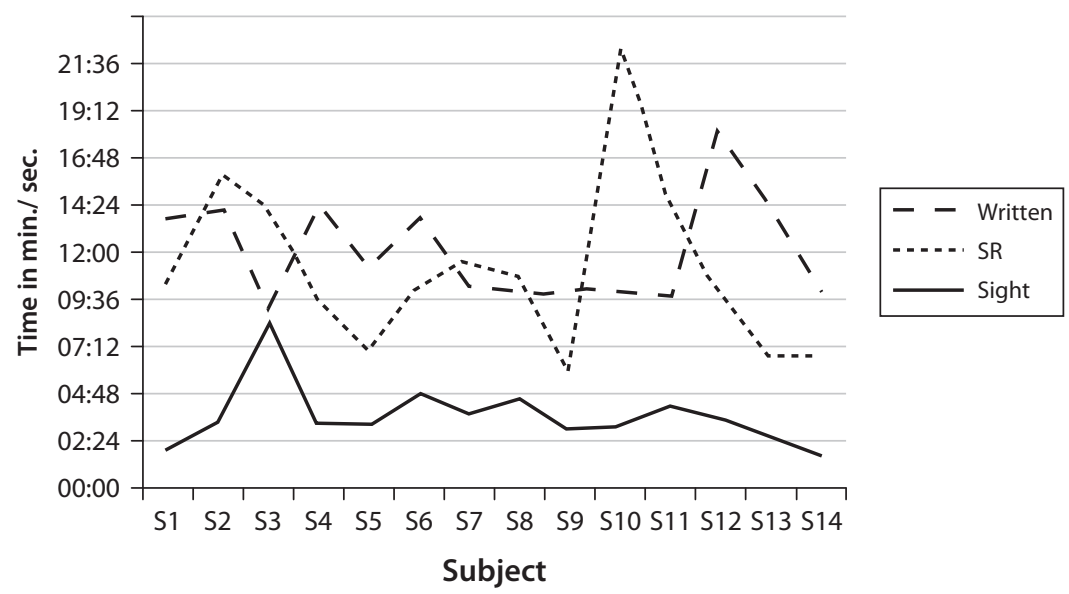

Figure 2. Individual task times in phase 2

\subsubsection{Number and types of misrecognitions (Research question 3)}

The number of misrecognitions in the experimental and control groups are presented in Table 3. The first phase exhibited 173 misrecognitions. The number of misrecognitions for the individual participants ranged from 4 to 32 . In phase 2 , the total number of misrecognitions increased to 248 , largely as a result of an increase in the experimental group. This comes as a surprise as we thought experience with the program would result in a decrease in the number of errors. For a possible explanation, we refer to the longer task times found particularly for those students who had taken the program home and learnt to use the oral commands, albeit ineffectively, and therefore spent more time attempting to correct errors than did the control group. Presumably this reflects a stage in the students' development, and we predict that once they have become more familiar with the software, or the software has adapted to their way of speaking, the number of errors will be substantially reduced.

The distribution of misrecognitions (Table 4) shows that the percentage of homophones remains more or less the same across time, the percentages of hesitations/word boundary problems and mispronunciations decrease whereas the relative number of errors attributable to the SR system increases in the second phase. The decrease in hesitations and word boundary problems could be caused by two factors. Firstly, the students had had far more interpreting training, which enhanced their oral competence and maybe also reduced the number of hesitations. A second possible reason is that even after very limited training with the program, they may simply have become better at dictating to the SR program and now realise how to avoid misrecognitions resulting from hesitations. The same may be true with regard to mispronunciations. Our retrospective interviews 
Table 3. Number of misrecognitions in the experimental and control groups - phases 1 and 2 compared

\begin{tabular}{lcl}
\hline Phase 1 & Misrecognitions & Range \\
\hline Experimental $(\mathrm{N}=7)$ & 75 & $4-18$ \\
Control $(\mathrm{N}=7)$ & 98 & $4-32$ \\
All students $(\mathrm{N}=14)$ & 173 & $4-32$ \\
\hline Phase 2 & Misrecognitions & Range \\
\hline Experimental $(\mathrm{N}=7)$ & 152 & $10-39$ \\
Control $(\mathrm{N}=7)$ & 96 & $2-30$ \\
All students $(\mathrm{N}=14)$ & 248 & $2-39$ \\
\hline
\end{tabular}

Table 4. Types of misrecognitions (means of 14 students) - phases 1 and 2 compared

\begin{tabular}{llllll}
\hline & Homophones & $\begin{array}{l}\text { Word boundaries } \\
\text { or hesitations }\end{array}$ & $\begin{array}{l}\text { Incorrect } \\
\text { pronunciations }\end{array}$ & $\begin{array}{l}\text { Inadequacy of } \\
\text { SR software }\end{array}$ & Total \\
\hline Phase 1 & $8 \%$ & $24 \%$ & $54 \%$ & $14 \%$ & $100 \%$ \\
Phase 2 & $6 \%$ & $14 \%$ & $38 \%$ & $42 \%$ & $100 \%$ \\
\hline
\end{tabular}

(Section 3.4) reveal that a number of students feel that they have become more aware of their pronunciation problems in the course of training the SR program. It is difficult to argue with a machine, and perhaps it is therefore easier to accept that one's pronunciation is inadequate in certain respects when the immediate consequence of an error is flashed back on a screen rather than when it is pointed out by a teacher.

\subsection{Quantitative conclusions}

In the first phase, there was a small time saving in translation with SR as compared with written translation. This does not increase after the training period. On the contrary: Our prediction of a reduction of task times in this modality was not confirmed. This is probably because the students who were given the program used it only very sparingly. In hindsight, we may have underestimated the follow-up and control procedures necessary to ensure that the students actually used the program to the extent we had prescribed. Most likely, more time, effort and guidance are required before improvements kick in and differences between the two groups can be registered. We feel convinced that productivity will improve with experience (Dragsted, Hansen and Sørensen 2009, 304), and that our measurements were collected at too early a stage in the students' development, at a point when their limited 
interaction with the program appeared to slow them down rather than speed them up. Secondly, the output quality of the SR translations was more similar to that of written translation; in fact, in certain cases, it was even better. The third conclusion was that the same four types of misrecognitions recurred in the second round of experiments but that the distribution was somewhat different, with relatively fewer hesitations/word boundary problems and mispronunciations in the second phase. We could observe a higher percentage of misrecognitions in the experimental group as compared with the control group. As we have seen earlier, this is probably owing to the students in the experimental group now practising the use of oral commands, and therefore struggling to correct errors in the second phase. Note, however, that there was great inter-individual variation in both groups.

\subsection{Retrospective interviews}

In the retrospective interviews, the students were asked what they thought of translating with SR versus the written modality. Repeatedly, they indicated that with SR, one obtains a better overall picture; one has to plan ahead what to say and this results in better quality; one doesn't waste time searching for the perfect solution; one doesn't translate word for word; and one doesn't look up and check everything. In other words, students appear to feel that a good strategy is to translate the message first and then work on honing the text later. When asked about the advantages, they said: it saved time; the process was quicker; it removed the "stop-go effect"; it was useful to see the text in print immediately; and to have their first impulse registered (cf. Chafe and Danielewicz 1987, 88). They also felt that both their spontaneous speech skills and their pronunciation had improved. When asked about the difficulties, the students stated that function words and numbers caused problems and also that the process was tiring. In addition, they thought certain texts were more appropriate for the use of speech recognition than others.

In due course, the students will become more aware of how to avoid misrecognitions and, with training, the system will adapt to the users' pronunciation and idiosyncrasies. Though not unimportant, remedying these formal errors is much less of an issue than students' misrepresentations of the content. It would therefore be fruitful to undertake qualitative analyses of both the process and the product following the procedure described in Göpferich (2010). By examining the quality of the solutions and the path to the solutions (i.e., the translation strategies adopted) in the different modalities, we will be able to see if the use of SR does indeed result in students translating larger meaningful units rather than individual words. 


\section{Discussion and conclusion}

We believe that our study has shown that SR translation is a possible alternative or supplement to other translation modalities in translator training. It would appear to be a powerful pedagogical tool to help translation students think in larger chunks and take a more panoramic view. In our opinion, this is the reason together with the additional T\&I training they had received in the intervening months - for the convergence of the quality ratings of written and SR translation in the second phase. A prominent feature in interpreting and other oral communication courses is to teach students how to plan their output and "think before they speak". The benefits of using SR could therefore also feed back into written translation if it teaches the students to adopt the motto "think before you write". It would appear that it helps them trust their first intuition rather than poring over isolated words and spending a disproportionate amount of time trying to solve a problem.

Students say that using the software raises their awareness of potential pronunciation errors and that speaking their translation encourages them to deal with larger units, and thus translate overall meaning instead of individual words. If universities ensure that subject areas such as oral proficiency, phonetics, translation and interpreting are integrated into courses more meaningfully, students will discover that, to use a familiar expression: "the whole is more than the sum of the parts”. An interdisciplinary approach will help Emma - our student who couldn't see the relevance of all the courses in her degree programme - appreciate the different components of the curriculum. So, rather than offering phonetics as an isolated subject, it would be preferable to link it to T\&I training, since the reasons for needing proper pronunciation become blatantly obvious once students find that interaction with the software only works if their pronunciation is accurate.

Our findings indicate that, with training, SR translation can probably be made faster and can attain a standard which is as high as that of written translation. This means that Peter, our translator with the broken arm, and other translators who are troubled by physical impairments may have a viable alternative to typing. But any translator may enjoy the mental and ergonomic variation of supplementing traditional keyboard activity with SR.

Not only translators, but anybody who produces text in a foreign language, may benefit from SR technology. For those who, like David, are dyslexic it is an obvious alternative, but more generally, anybody who finds it challenging to deal with written language, notably in a foreign language, may find it an advantage to make a quick first draft with an SR system (Leijten, Janssen and Van Waes 2010, 965). Having problems with writing or spelling does not imply that one necessarily has problems with speaking and pronouncing. 
The readiness to embrace new technologies is an important prerequisite for succeeding with the SR program. Translators who have been practising for many years will have to invest considerable time and energy in order to fundamentally change their working habits and take on new tools. Universities have an important role to play in introducing SR in addition to other technologies as part of the translator's toolbox. For too long universities like our own have engaged in traditional approaches to translator training, and have not addressed the changing preferences of students for obtaining and communicating information. Universities have tended to ignore the fact that young people read less than former generations, and that they are simply no longer attracted by black print on white paper. They are multimodal and expect classes in translation and other subjects to be more interactive and to incorporate media dealing with text, audio, animation and video (Hansen and Shlesinger 2007, 111). With the recent development of voice activation in smart phones and computers there is a golden opportunity to capture students' interest. As speech becomes a more reliable and popular input method, using SR may grow into a more natural working mode also in translation. In sum, curricula have to be made more interdisciplinary to prepare students for life as translators, where they have to be able to use a wide array of technological solutions that may enhance their performance.

Altogether, SR software can be seen as a tool for translation research, teaching and practice. We hope to have shown the sound effects of using it.

\section{Dedication}

This chapter is dedicated to the memory of Miriam Shlesinger (1947-2012) - leading scholar and close friend. In her capacity as Honorary Doctor at CBS (our home institution), she assisted us in numerous matters big and small. When presented with a number of different titles for this contribution, she replied: “Sound effects in translation' is my favorite". Miriam was our favourite.

\section{Acknowledgement}

We are very grateful to two anonymous reviewers for their insightful comments and many useful suggestions. 


\section{References}

Agrifoglio, Marjorie. 2004. "Sight Translation and Interpreting: A Comparative Analysis of Constraints and Failures." Interpreting 6 (1): 43-67. DOI: 10.1075/intp.6.1.05agr

Chafe, Wallace, and Jane Danielewicz. 1987. "Properties of Spoken and Written Language." In Comprehending Oral and Written Language, ed. by Rosalind Horowitz, and S. Jay Samuels, 83-113. San Diego: Academic Press.

Collins, Beverley, and Inger M. Mees. 2013. Practical Phonetics and Phonology, 3rd ed. Abingdon: Routledge.

Cruttenden, Alan. 1994. Gimson's Pronunciation of English, 5th ed. London: Arnold.

Derwing, Tracey M., Murray J. Munro, and Michael Carbonaro. 2000. "Does Popular Speech Recognition Software Work with ESL Speech?” TESOL Quarterly 34 (3): 592-603.

DOI: $10.2307 / 3587748$

Dragsted, Barbara, Inge Gorm Hansen, and Henrik Selsøe Sørensen. 2009. “Experts Exposed.” In Methodology, Technology and Innovation in Translation Process Research [Copenhagen Studies in Language, 38], ed. by Inger M. Mees, Fabio Alves, and Susanne Göpferich, 293317. Copenhagen: Samfundslitteratur.

Dragsted, Barbara, Inger M. Mees, and Inge Gorm Hansen. 2011. "Speaking Your Translation: Students' First Encounter with Speech Recognition Technology." Translation \& Interpreting 3 (1): 10-43.

Gile, Daniel. 1995. Basic Concepts and Models for Interpreter and Translator Training. Amsterdam: John Benjamins. DOI: 10.1075/btl.8

Gile, Daniel. 2004. "Translation Research Versus Interpreting Research: Kinship, Differences and Prospects for Partnership." In Translation Research and Interpreting Research: Traditions, Gaps and Synergies, ed. by Christina Schäffner, 10-34. Clevedon: Multilingual Matters.

Göpferich, Susanne. 2010. "The Translation of Instructive Texts from a Cognitive Perspective: Novices and Professionals Compared." In New Approaches in Translation Process Research [Copenhagen Studies in Language, 39], ed. by Susanne Göpferich, Fabio Alves, and Inger M. Mees, 5-55. Copenhagen: Samfundslitteratur.

Hansen, Inge Gorm, and Miriam Shlesinger. 2007. “The Silver Lining: Technology and Self-Study in the Interpreting Classroom." Interpreting 9 (1): 95-118. DOI: 10.1075/intp.9.1.06gor

Jakobsen, Arnt Lykke, and Lasse Schou. 1999. “Translog Documentation.” In Probing the Process in Translation: Methods and Results [Copenhagen Studies in Language, 24], ed. by Gyde Hansen, 151-186. Copenhagen: Samfundslitteratur.

Jurafsky, Daniel, and James H. Martin. 2000. Speech and Language Processing: An Introduction to Natural Language Processing, Computational Linguistics, and Speech Recognition. New Jersey: Prentice Hall.

Lambert, Sylvie. 2004. "Shared Attention during Sight Translation, Sight Interpretation and Simultaneous Interpretation." Meta 49 (2): 294-306. DOI: 10.7202/009352ar

Leijten, Mariëlle, Daniel Janssen, and Luuk Van Waes. 2010. "Error Correction Strategies of Professional Speech Recognition Users: Three Profiles." Computers in Human Behavior 26: 964-975. DOI: 10.1016/j.chb.2010.02.010

Leijten, Mariëlle, and Luuk Van Waes. 2005. "Writing with Speech Recognition: The Adaptation Process of Professional Writers with and without Dictating Experience." Interacting with Computers 17: 736-772. DOI: 10.1016/j.intcom.2005.01.005 
Luyckx, Bieke, Tijs Delbeke, Luuk van Waes, Mariëlle Leijken, and Aline Remael. 2010. "Live Subtitling with Speech Recognition: Causes and Consequences of Text Reduction." Artesis Working Papers in Translation Studies 1. Antwerp.

Phillipson, Robert. 2003. English-Only Europe: Challenging Language Policy? London: Routledge. Wells, John C. 2008. Longman Dictionary of Pronunciation, 3rd ed. Harlow: Pearson Education. DOI: $10.1177 / 003368829002100208$

Zong, Chengqing, and Mark Seligman. 2005. “Toward Practical Spoken Language Translation.” Machine Translation 19 (2): 113-137. DOI: 10.1007/s10590-006-9000-Z 



\section{Subject index}

\section{A}

actant 38

activity theory 39

actor-network theory 38

Adhoc Block 52, 118

analytical phase 101

ANT see actor-network

theory

archive 129,130

artefact analysis $38,41,42$

attractor state $65,72,74,75$

automatized skills $64,69,76$

\section{B}

bilingualism research 7,77

C

cognition 9, 10, 36, 37, 38, 39,

43,44

see also situated cognition, embodied cognition,

distributed cognition

cognitive apprenticeship 75

cognitive efficiency 121

cognitive effort $7,12,19,64$,

$69,70,72,73,74,103,104$,

108

cognitive flow 118, 120, 121,

122

cognitive load $9,10,11,19,20$,

$22,23,24,25,26,27,28,29$,

30, 104, 106

Cognitive Load Model 22,

23, 24

cognitive overload $27,28,120$

cognitive science $9,10,12,35$,

$36,37,52,60,110,124$

Cognitive Translation

Hypothesis 100, 101

cognitive translatology $6,7,8$,

$9,10,11,12,13$

community of practice 89,91 computer logging see

keystroke logging

computer-supported

cooperative work 39,41

conceptual encodings 110,

$114,115,116,117,120$

conceptual metaphor 96,97 ,

102, 104, 105, 106

concurrent verbalization 20 ,

$21,24,81$

connected growers 76

consciousness 52

constructed decisions 70

coping skills 75

corpus linguistics $6,7,110,137$

covert translation $58,59,60$

creativity $69,71,72,74,75$,

100, 101, 103

creativity research 100

creativity/routine profile 69 ,

$71,72,74$

crowd sourcing 8

CSCW see computersupported cooperative work

cue-based retrospection 81 , 82

cultural filter 59

culture $36,37,40,43,44$,

45,51

see also translation culture

\section{D}

decision making $68,69,70$, $72,74,76,89,91,97,100$, 108,123

decision-making typology 69,70

declarative behaviour 52 declarative knowledge 75

deliberate practice $9,74,75$ descriptive translation studies 136

developmental hypothesis 76

dialogue protocol 100, 101

difficulty $7,24,25,29,145$

distributed cognition 35,37 , 38,40

Dragon Naturally Speaking 143

DST see Dynamic Systems Theory

dynamic system $\quad 66,72,75$

Dynamic Systems Theory 63 , $64,65,66,70,74,75,76,77$

E

EEG see electroencephalography

Effort Model 22, 23, 24

effort models 21, 64, 75

electroencephalography 9, 26

embodied cognition 10,37

enrolment 39

ERP see event-related potential

ethnography $13,36,38,39,41$, $43,80,91$

ethnomethodology 39

evaluation competence 72,75

event-related potential 54

expertise $8,9,11,12,13,45$,

72,74

expertise studies see expertise extended cognition 37,38 ,

41,43

extended translation 43

eye tracking $5,9,10,11,12,28$,

$53,79,81,84,88,100,103$

$104,105,107,124,137,143$

eye-mind coordination 10

eye-mind hypothesis 10, 11 
F

feedback 74,75

foci 53,131

fRMI see functional

Magnetic Resonance

Imaging

functional Magnetic

Resonance Imaging 9,

27,54

\section{G}

guessing $68,69,70$

\section{$\mathrm{H}$}

high-effort decision 72

homophones $146,147,149$, 150

human-computer interaction 12,13

\section{I}

ICT 42,43

inferential processing 110, 112

information and

communication

technologies 42

InputLog 85

interessement 39

introspection 20, 52, 104

invisibility 50

\section{K}

keystroke logging 10, 11, 53, $54,67,80,81,84,85,100$, $101,104,137,143$

\section{L}

L2 competence 70

language acquisition 64,65 , 66,76

language competence 75 , 76, 91

language technology 6,11

liberal arts paradigm $\quad 128$

linguistic-cognitive translation theory 50

long-term working memory 76

longitudinal study $45,63,64$, $66,67,76,80,83,90$

low-effort decision 72
M

machine translation 10, 11, 12,14

macro translation unit 115 , $116,117,119,122,124$

macro TU see macro

translation unit

macro-strategy 68

mapping $30,96,97,98$

memory capacity $10,12,64$,

$68,69,70,75,76$

meta-cognitive competence 68

metacognition 9, 11

metacognitive monitoring

52,118

metaphor identification 101

metaphor interpretation 101, 103, 105

metaphor studies 96, 97, 106

micro translation unit 115 ,

$116,117,119,120,121,122$,

123, 124

micro TU see micro

translation unit

mispronunciation 149,151

misrecognitions $142,144,146$,

$147,149,150,151$

motivation $57,67,68,74$,

131, 143

MT see machine translation

multilingual approach 98,105

Multiple Resource Theory 22

$\mathrm{N}$

nesting 65

networking 43

neuro-linguistic studies 56 ,

60

neuroscience $6,9,12,32,52$

newswriting research 80,82 nexus 133

non-fixedness 69

norms $37,44,57,59,66,67$,

$98,99,128,136$

novice-expert paradigm 63

O

observer's paradox 52

oral translation 141

overt translation $58,59,60$
$P$

performance accuracy 25

performance methods 20 ,

21, 25, 26

performance speed 25

personal papers 129

personality 8

PET see positron emission

tomography

phonetics 142, 152

polysemy 7

positron emission tomography

26,54

post-editing 12

post-hoc verbalization 24

preliminary norms 128

see also norms

principle of relevance 111,112

problem awareness 75,76

problem solving $44,51,64$,

$69,70,75,80,91,109,118$,

$119,122,123,131$

problem-solving competence 75

procedural encodings 110,

$115,116,117,119,120,121$,

$122,123,124$

proceduralization 75,76

profiling $7,8,12$

progression analysis 82,83 ,

$84,85,89,91$

progression graph 87,88

project management system

42

pronunciation $142,144,146$,

$147,150,151,152$

psycho-physiological methods

20, 21

psycholinguistics 7

psychology $7,25,35,38,64$,

$77,81,100$

psychometric profiling 8

psychometrics 8,13

pupil dilation $10,27,28,29$

pupillometry $20,27,28,29$

R

readability 7,11

reflected decisions 70

regressions 116

relevance theory 110, 111, 112,

$113,114,115,116,117,121$

repeller state 65 
Rest Block 52, 118

retrospective verbal protocols

$82,83,89,91$

Rhetorical Structure Theory

$7,13,114$

routine $51,58,64,68,69,70$,

$71,72,74,75,76$

routinized decisions 70

RST see Rhetorical Structure

Theory

RT see relevance theory

RVPs see retrospective verbal protocols

\section{$S$}

S-notation $86,87,88$

scenes-and-frames semantics 101

screen capturing see screen recording

screen recording $5,8,10,11$, $53,67,82,84,89$

self-report 20, 24, 81

shifts $43,51,53,64,68,69,75$, $76,107,136$

sight translation 10, 25, 142,

$143,145,147,148$

signal-to-noise ratio 30

situated and distributed

cognition see situated

cognition, distributed

cognition

situated cognition $36,37,39$,

$41,43,44,45,46$

situative approach 35

specialisation $41,42,43$

speech recognition 142,143 ,

$144,145,146,147,148,149$,

150, 151, 152, 153

SR see speech recognition

stagnation $65,70,74,75,76$

stereotype decisions 70

strategic competence 64,67 ,

$68,69,72,75$ strategy application $\quad 64,65$

subject profiling 16

subjective methods 20, 24, 25

switch competence 71

synthesis phase 101

systemic-functional

translation theory 49

\section{$\mathrm{T}$}

TA see think-aloud

TAP see think-aloud protocol text profiling 7,8

think-aloud $67,78,100,101$, 131

think-aloud protocol 37,100 , $131,136,137$

TM see translation memory (system)

tools and research competence 67,68

TransComp 66, 67, 68, 69, $70,72,76$

translation competence 63 , $64,65,66,67,72,74,75,76$, 77,79

translation competence development $63,65,66,75$ translation competence model 64,67

translation culture 36,43 , 44,45

translation difficulty 7

translation in the wild 36

translation management 41

translation memory (system) $10,11,12,42,43,44,86$

translation modalities 144 , 152

translation norms see norms translation product 66,67 , 69, 76, 90, 100, 127

translation quality $8,12,75$, 144 translation routine activation competence $58,67,68$

translation strategies $9,57,83$, 103, 104, 106, 130, 131, 151

translation unit $7,9,52,75$, 110, 115, 117, 119

translational norm see norms translatology $5,6,7,8,9,10$, $11,12,13$

translator papers $127,129,136$ translator profiling 12

translator training $8,143,144$, 152,153

Translog 101, 102, 103, 104, $114,115,144$

transposition 68

triangulation $10,105,137$

$\mathrm{U}$

uncertainty 9, 100, 104, 133

universals of translation 98

V

verbal data 82,104

verbal protocol $8,9,10,52,82$ verbal report data see verbal protocol

version analysis $81,83,89,90$ volunteer translation 8

W

WAIC see Wechsler Adult Intelligence Scale

Wechsler Adult Intelligence Scale 8 working memory (capacity) $10,52,64,68,69,70,75,76$ writing research $6,11,80,87$ written translation 142,144 , $145,146,147,148,150,151$, 152 